\title{
Magnetic and structural properties of isolated and assembled clusters ${ }^{\text {ts }}$
}

J. Bansmann ${ }^{\mathrm{a}, *}$, S.H. Baker ${ }^{\mathrm{b}}$, C. Binns ${ }^{\mathrm{b}}$, J.A. Blackman ${ }^{\mathrm{c}}$, J.-P. Bucher ${ }^{\text {, }}$, J. Dorantes-Dávila ${ }^{\mathrm{e}}$, V. Dupuis ${ }^{\mathrm{f}}$, L. Favre ${ }^{\mathrm{f}}$, D. Kechrakos ${ }^{\mathrm{g}}$, A. Kleibert ${ }^{\mathrm{a}}$, K.-H. Meiwes-Broer ${ }^{\mathrm{a}}$, G.M. Pastor ${ }^{\mathrm{e}}$, A. Perez ${ }^{\mathrm{f}}$, O. Toulemonde ${ }^{\mathrm{d}}$, K.N. Trohidou ${ }^{\mathrm{g}}$, J. Tuaillon ${ }^{\mathrm{f}}, \mathrm{Y} . \mathrm{Xie}^{\mathrm{c}}$

anstitut für Physik, Universität Rostock, Universitatsplatz 3, D-18051 Rostock, Germany

${ }^{\mathrm{b}}$ Department of Physics and Astronomy, University of Leicester, Leicester LE1 7RH, UK

${ }^{\mathrm{c}}$ Physics Department, University of Reading, Reading RG6 6AF, UK

${ }^{\mathrm{d}}$ Institut de Physique et Chimie des Matériaux de Strasbourg (IPCMS), UMR 7504 CNRS, Université Louis Pasteur, F-67037 Strasbourg, France

${ }^{\mathrm{e}}$ Laboratoire de Physique Quantique, CNRS, Université Paul Sabatier, F-31062 Toulouse Cedex, France

${ }^{\mathrm{f}}$ Laboratoire de Physique de la Matiere Condensée et Nanostructures, Université Claude Bernard, Lyon 1 and CNRS, F-69622 Villeurbanne Cedex, France

${ }^{\mathrm{g}}$ Institute of Materials Science, National Center for Research “Demokritos”, Attiki, Greece

Available online 22 December 2004

\begin{abstract}
Within the last years, a fundamental understanding of nanoscaled materials has become a tremendous challenge for any technical applications. For magnetic nanoparticles, the research is stimulated by the effort to overcome the superparamagnetic limit in magnetic storage devices. The physical properties of small particles and clusters in the gas phase, which are considered as possible building blocks for magnetic storage devices, are usually sizedependent and clearly differ from both the atom and bulk material. For any technical applications, however, the clusters must be deposited on surfaces or embedded in matrices. The contact to the environment again changes their properties significantly. Here, we will mainly focus on the fundamental electronic and magnetic properties of metal clusters deposited on surfaces and in matrices. This, of course, requires a well-defined control on the production of nanoparticles including knowledge about their structural behaviour on surfaces that is directly related to their
\end{abstract}

\footnotetext{
is This work is based on results of the EU "AMMARE" project within the Fifth Framework programme coordinated by Antonis N. Andriotis, Heraklion, Greece.

* Corresponding author.

E-mail address: joachim.bansmann@physik.uni-rostock.de (J. Bansmann).
} 
magnetic properties. We describe two different approaches to produce magnetic nanoparticles: (i) cluster aggregation on reconstructed single crystal surfaces and (ii) deposition of mass-filtered clusters from the gas phase onto surfaces and into matrices. The process of cluster deposition offers the possibility of creating new materials in non-equilibrium conditions with tailored properties.

A theoretical description of the evolution of cluster magnetism is given with respect to contributions from magnetic anisotropy effects and the local atomic environment. Especially small clusters show size-dependent magnetic orbital and spin moments that can experimentally be accessed by the element-specific technique of X-ray magnetic circular dichroism (XMCD). The magnetic properties of self-organized iron and cobalt clusters on $\mathrm{Au}\left(\begin{array}{lll}1 & 1 & 1\end{array}\right)$ surfaces are discussed with respect to growth conditions. For deposited Fe clusters on surfaces increased orbital moments have been found even for large particles. Additionally, the influence of capping layers and deposition into matrices is discussed.

While investigations on relative simple structures in pure $3 \mathrm{~d}$ metal particles yield insight into the basic mechanisms of magnetism, alloy nanoparticles seem to be more promising in terms of technical application since they offer the possibility to adjust the magnetic properties by varying the stoichiometry. Alloys consisting of $3 \mathrm{~d}$ metals (e.g. $\mathrm{Fe}_{x} \mathrm{Co}_{1-x}$ alloys) have usually very high magnetic moments and are soft-magnetic. Binary clusters consisting of a $3 \mathrm{~d}$ metal (e.g. Co) in combination with a heavy element ( $\mathrm{Sm}, \mathrm{Ag}$ or Pt) are candidates for materials with high magnetic anisotropies and increased blocking temperatures. The magnetic properties are directly related to their structural order. Here, we show first results for such alloy nanoparticles.

(C) 2004 Elsevier B.V. All rights reserved.

PACS: 73.22.-f; 75.75.+a; 81.07.-b

Keywords: Clusters on surface; Magnetism; Iron; Cobalt; Binary alloy clusters

\section{Contents}

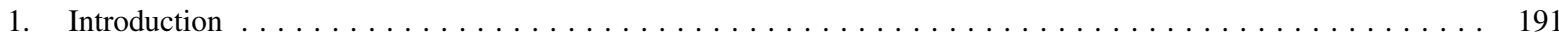

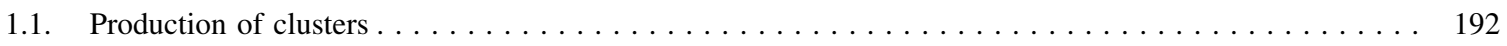

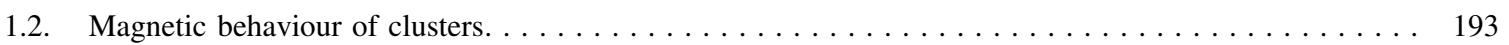

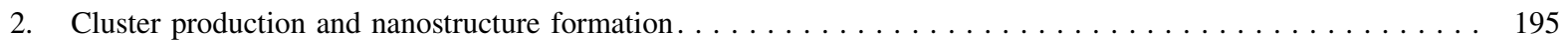

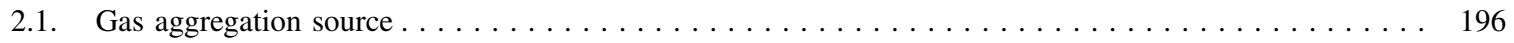

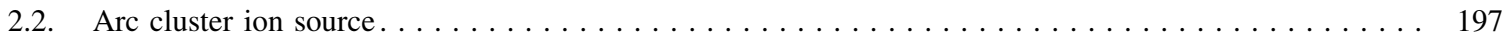

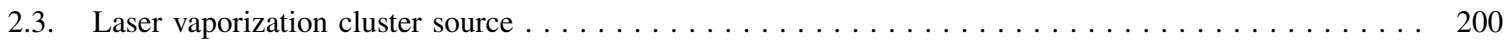

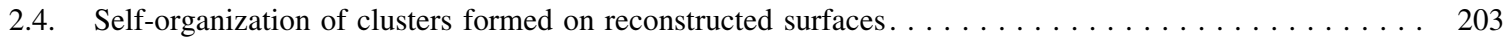

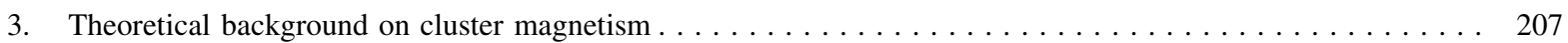

3.1. Model Hamiltonians . . . . . . . . . . . . . . . . . . . . . . . . . . . . . . . . . . . . 207

3.2. Local atomic environment and spin magnetic moments. . . . . . . . . . . . . . . . . . . . . 209

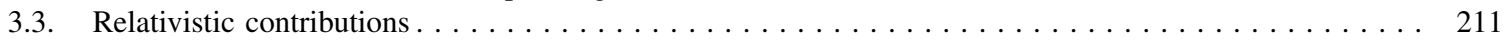

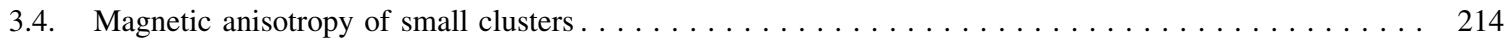

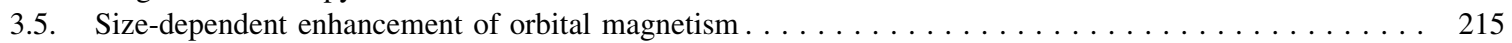

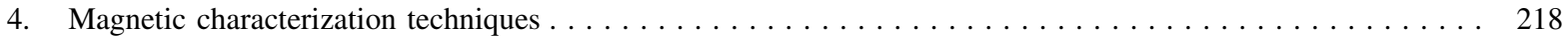

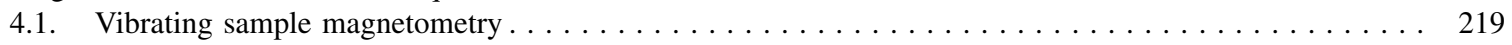

4.2. Magneto-optical Kerr effect in the visible regime. . . . . . . . . . . . . . . . . . . . . . . 219

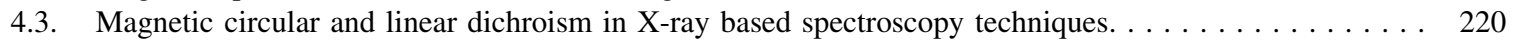

4.3.1. X-ray magnetic circular dichroism in photoabsorption $\ldots \ldots \ldots \ldots \ldots \ldots \ldots \ldots \ldots \ldots 221$

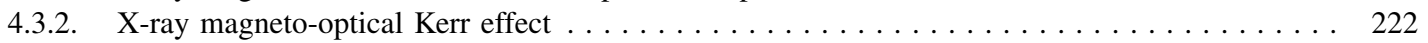




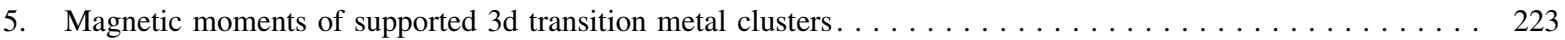

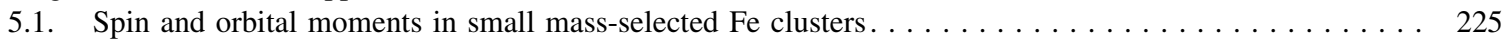

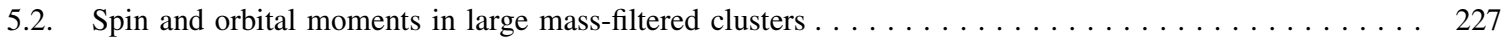

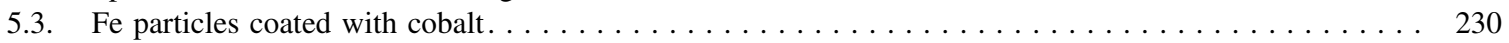

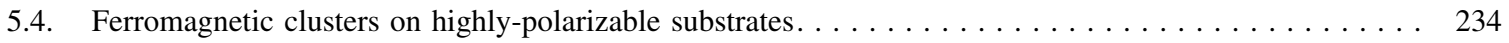

5.4.1. Spin and orbital moments of $\mathrm{Co}_{N}$ on $\operatorname{Pd}\left(\begin{array}{lll}1 & 1 & 1\end{array}\right) \ldots \ldots \ldots \ldots \ldots \ldots$

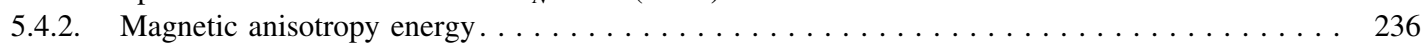

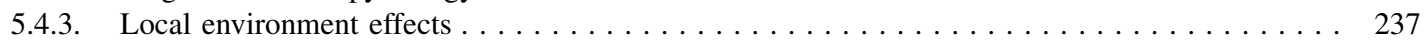

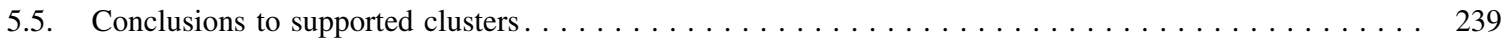

6. Magnetic behaviour of transition metal clusters in matrices $\ldots \ldots \ldots \ldots$

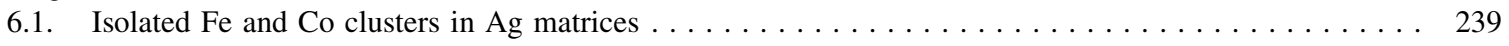

6.2. Magnetic behaviour of interacting cluster assemblies $\ldots \ldots \ldots \ldots \ldots \ldots \ldots$

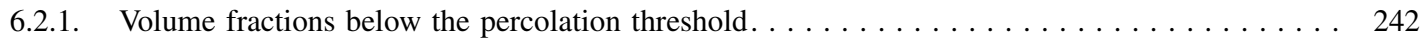

6.2.2. Volume fractions above the percolation threshold . . . . . . . . . . . . . . . . . 243

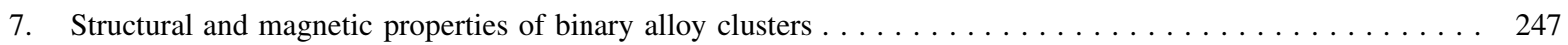

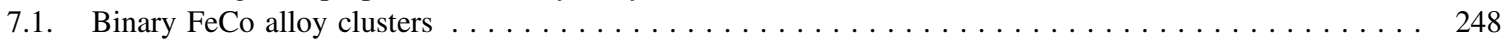

7.2. Structural and magnetic properties of CoSm clusters $\ldots \ldots \ldots \ldots \ldots \ldots$

7.3. Structural and magnetic properties of mixed $\mathrm{Co}-\mathrm{Ag}$ and $\mathrm{Co}-\mathrm{Pt}$ clusters $\ldots \ldots \ldots \ldots$

7.4. Spin moments, orbital moments, and magnetic anisotropy of Co-Rh clusters $\ldots \ldots \ldots 255$

7.4.1. First principles calculations of structure and spin moments of small $\mathrm{Co}_{N} \mathrm{Rh}_{M}$ clusters $\ldots \ldots 256$

7.4.2. Spin moments, orbital moments and magnetic anisotropy energy of large $\mathrm{Co}_{N} \mathrm{Rh}_{M}$ clusters. . . 257

8. Self-organized $\mathrm{Fe}$ and $\mathrm{Co}$ clusters on $\mathrm{Au}\left(\begin{array}{lll}1 & 1 & 1\end{array}\right) \ldots \ldots \ldots \ldots \ldots$

8.1. Assembly of perpendicularly magnetized clusters on a regular network $\ldots \ldots \ldots 260$

8.2. The effect of cluster coalescence: $\mathrm{Fe} / \mathrm{Au}\left(\begin{array}{lll}1 & 1 & 1\end{array}\right) \ldots \ldots \ldots \ldots \ldots \ldots \ldots$

8.3. Interacting islands; from $1 \mathrm{D}$ chains to $2 \mathrm{D}$ magnetism $\ldots \ldots \ldots \ldots \ldots$

8.4. In-plane anisotropy induced by low temperature growth $\ldots \ldots \ldots \ldots \ldots$

8.5. Magnetic tuning through temperature induced segregation of binary clusters $\ldots \ldots \ldots 6$

9. Conclusion and outlook $\ldots \ldots \ldots \ldots \ldots \ldots \ldots$

Acknowledgements . . . . . . . . . . . . . . . . . . . . . . . . . . . . . . 269

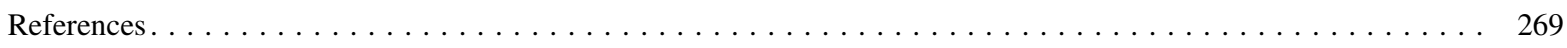

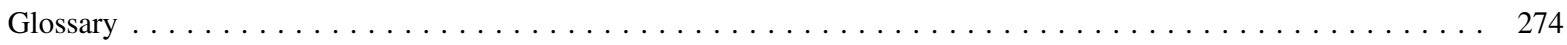

\section{Introduction}

Free clusters and nanoparticles are not just small pieces of material with physical properties nearly identical to the bulk. Their electronic, optical and magnetic properties are clearly size-dependent with a non-linear behaviour between the two general limits given by the atomic and the bulk-like behaviour. Thus, cluster physics is related to the evolution of the fundamental physical properties of a material as it is built up atom by atom (for overviews, see [1-4]). The clusters themselves can be considered as functionalized building blocks whose properties can be selected by controlling their size. In addition, there is a growing realization of the enormous potential of assemblies of supported nanoclusters in the production of high-performance magnetic materials and devices. Technical applications of these new 
materials require the deposition on surfaces or into matrices, which often changes the properties of free clusters by the particle-support interaction (e.g. hybridization, changes in the geometry upon deposition, diffusion, alloy formation) and, in the case of high coverages, by particle-particle interaction. The motivation for studying mass-selected magnetic nanoclusters is the unique opportunity to observe how magnetism develops as a material is built from individual atoms to the solid state.

Most of the atomic scale magnetic structures have been created by atom agglomeration on epitaxial, sometimes reconstructed, surfaces. Typical examples are Co clusters on $\operatorname{Au}\left(\begin{array}{lll}1 & 1 & 1\end{array}\right)$ [5] and $\operatorname{Pt}\left(99^{9} 7\right)$ [6] surfaces as well as Fe particles on $\mathrm{Cu}\left(\begin{array}{lll}1 & 1 & 1\end{array}\right)$ [7], $\mathrm{Mo}\left(\begin{array}{lll}1 & 1 & 0\end{array}\right)$ [8] and W(lll 110$)$ surfaces [9]. For example, Gambardella et al. [6] observed very large orbital moments (up to $1.1 \mu_{\mathrm{B}}$ per atom) for Co nanoparticles with a few number of atoms on a platinum surface. The data nicely show the decrease of the orbital moment towards the bulk value with increasing cluster size. Time-resolved spin-polarized STM measurements on nanoscaled Fe clusters on Mo( 1110$)$ clearly show the superparamagnetic switching in individual islands. These highly exciting experimental results are restricted to very small structures and low temperatures. Experiments on free, mass-selected 3d metal clusters also showed striking size effects and strongly enhanced total magnetic moments [10]. An open question is whether or not such effects can still be observed when clusters from the gas phase are deposited on surfaces or into matrices. Moreover, it will be important to determine up to which cluster size these interesting phenomena with potential technical applications can be observed. The aim here is to give an overview of the magnetic phenomena of deposited clusters in contact with surfaces in comparison to the behaviour of thin films and bulk-like materials. Moreover, the article outlines recent approaches in cluster physics to extend the fascinating results on atomic scale structure to larger cluster sizes and to higher temperatures.

\subsection{Production of clusters}

The starting point in experimental research related to cluster physics is the possibility to produce these nanoparticles with a well-defined number of atoms. Today, different kinds of approaches exist for producing nanostructures and nanoparticles on surfaces. Nanostructures can be created sequentially by electron beam lithography, focused ion beam (FIB) techniques and related methods. Recently, wet chemical processes were invented to produce, e.g. FePt clusters with a narrow size distribution covered with an organic ligand shell in solutions. These nanoparticles form well-ordered structures on surfaces within a lateral regime of several $\mu \mathrm{m}$ [11] and thus have a huge potential for technical applications $[12,13]$. However, further chemical processes are necessary to remove the ligands and to obtain optimized physical properties since the particles are usually oxidized, at least after removing the outer ligand shell by, e.g. plasma etching. For fundamental research, one possible way of obtaining nanoparticles on surfaces is the deposition of atoms on carefully controlled substrates with well-defined nucleation sites or thin oxide film given rise to cluster growth. Such sites can be formed, for example, by surface reconstructions (herringbone reconstruction on $\operatorname{Au}\left(\begin{array}{lll}1 & 1 & 1\end{array}\right), \operatorname{Ag}$ overlayer on $\operatorname{Pt}\left(\begin{array}{lll}1 & 1 & 1\end{array}\right)$ [14]) or artificially created defects on HOPG and $\mathrm{Cu}\left(\begin{array}{lll}1 & 0 & 0\end{array}\right)$. Details on cluster growth by atom aggregation are given in several review articles (e.g. [3,4,15]). A short description of the cluster formation on $\mathrm{Au}\left(\begin{array}{lll}1 & 1 & 1\end{array}\right)$ is given in Section 2.4. As an alternative to this process, which dramatically depends on the properties of the substrate and its interaction with the deposited atoms, one can nucleate the particles as free clusters and finally deposit them under soft-landing conditions on surfaces. This approach has the advantage that the size of the clusters, as well as their structure to a certain extent, can be controlled prior to deposition. The technique has the potential of creating new materials with tailored properties. Since the clusters are 
generated under non-equilibrium conditions it is possible to create metastable structures or alloys which cannot be fabricated by atom aggregation on surfaces. Moreover, upon deposition on surfaces the clusters must transfer their kinetic energy to the substrate to ensure efficient sticking. The energy dissipation depends mainly on the relation between internal cluster binding strength and on the cluster impact energy (see [16]). Here, we will focus on the low-energy impact regime, which is characterized by kinetic energies of about $0.1-1 \mathrm{eV}$ per atom. Clusters deposited on different substrates may adopt different geometrical shapes and structures depending on the nature of the substrate.

\subsection{Magnetic behaviour of clusters}

The main sources of magnetism in atoms, clusters and solids are the currents associated with the electronic motion (orbital magnetism) and the intrinsic angular momentum of the electrons (spin magnetism). These properties have a quantum mechanical nature and cannot be regarded as the superposition of independent individual electron contributions. They result from the behaviour of the ensemble of electrons in the relevant low-energy scale (valence electrons). Consequently, the magnetic properties of matter are very sensitive to the details of the electronic correlations and to temperature.

In isolated atoms almost all elements show a non-vanishing magnetic moment given by Hund's rules, while in the solid state only a few of them (some transition metals of the Fe group, the lanthanides and actinides) preserve a non-vanishing magnetization. The latter is a consequence of the delocalization of the valence electrons, which favours equal occupation of states having opposite projections of spin and orbital moments. Finite clusters constitute a new state of matter with its own fascinating characteristics. From this perspective, the properties of atoms and solids may be regarded as limiting cases of a much richer and complex dependence on system size. One of the main goals in cluster theory is to understand how the properties of the valence electrons of atoms change as they become part of a small particle and start to delocalize. The magnetism of transition metal (TM) clusters constitutes one of the fundamental challenges, since atomic and bulk behaviours are intrinsically different. Atomic magnetism is due to electrons that occupy localized orbitals, while in TM solids the electrons responsible for magnetism are itinerant, conducting d-electrons. Consequently, the magnetic properties of nanoparticles are very sensitive to size, composition, and local atomic environment thus showing a wide variety of intriguing phenomena.

It has been known for over a decade that free nanoclusters have novel magnetic properties. Magnetic phenomena are very sensitive to the details of the atomic environment. First calculations on the electronic structure and magnetic properties of small iron and nickel clusters started already in the 1980s. Lee et al. [17] proposed a narrowing of the d-bands with decreasing number of atoms in Fe clusters and an enhanced spin polarization in Fe clusters compared to the bulk. Shortly later, Pastor et al. [18] extended these calculations significantly, including different geometric structures in the size range from 2 atoms per cluster up to about 50 atoms. Very small Fe clusters with less than 10 atoms showed magnetic moments of about $3 \mu_{\mathrm{B}}$ with decreasing values for larger clusters. The first measurements on magnetic phenomena of $3 \mathrm{~d}$ metal clusters in a molecular beam were performed in the beginning of the 1990s by the groups of de Heer [10,19,20] and Bloomfield [21-24]. Both groups used inhomogeneous magnetic fields (Stern-Gerlach magnets) to explore the magnetic moments by deflecting the superparamagnetic clusters. It turned out that the temperature of the clusters, being a crucial parameter in investigating magnetic properties, is difficult to determine and opened a discussion on the experimental results. For free massselected clusters, it is possible to observe how the magnetic properties change from the monomer to the bulk [10,21]. These include a significant increase in the magnetic moment per atom relative to the bulk in 
3d transition metals [10,20,23], the appearance of magnetism in paramagnetic metals [25] and ferrimagnetism in antiferromagnetic materials [26]. Lowered magnetic moments per atom in ferromagnetic rare-earths have been ascribed to canted atomic moments [23,27] and both lowered [20,28] and increased [29] Curie temperatures have been observed. Particularly large changes are observed in very small clusters; Knickelbein [30], for example, observed a magnetic moment per atom very close to the atomic limit of $6 \mu_{\mathrm{B}}$ per atom in 12-atom clusters that reduces to a value close to the bulk limit of $2.2 \mu_{\mathrm{B}}$ per atom on addition of a single atom to produce a 13-atom cluster.

This rich variety of behaviour can be understood in general terms as arising from the reduced average coordination per atom, the quantum size effect and modified collective electron behaviour such as screening and charge spill-out from the surface. Theoretical models have been developed that explain many of these phenomena in detail. Ab initio methods and total energy calculations can be used to determine the cluster structure as well as their magnetic properties but are restricted to small clusters containing a few tens of atoms. Hartree-Fock based methods can be used to calculate the magnetic behaviour of much larger clusters but an atomic structure must be assumed. It is found in all cases that the atomic structure and the nearest-neighbour distances are critical to determine the magnetic properties in clusters.

The average coordination number $\langle z\rangle$ is a key parameter that controls the evolution of magnetic behaviour. In transition metals magnetism is dominated by the spin moment $m_{\mathrm{S}}$. Reducing the dimension leads to a narrowing of the valence $d$ bands that generate most of the magnetic moment. The increase in the density of states at the Fermi level $E_{\mathrm{F}}$ also opens the chance to detect magnetic phenomena in materials which are non-magnetic in bulk (e.g. Ru monolayers [31] and 4d clusters on $\operatorname{Ag}\left(\begin{array}{lll}0 & 0 & 1\end{array}\right)$ [32]). Itinerant magnetic materials have an orbital magnetic moment $m_{\mathrm{L}}$ induced by spin-orbit coupling. The general trend is the same one as for the spin moment, the orbital moment increases with decreasing coordination. However, the orbital moment does not linearly follow the spin moment, the ratio $m_{\mathrm{L}} / m_{\mathrm{S}}$ varies with atomic structure, dimensionality and coordination number [33]. In the case of free atoms, the orbital and spin moment can be calculated by Hund's rules in atomic physics. For Fe having six electrons in the d-shell, we gain values of $m_{\mathrm{S}}=4 \mu_{\mathrm{B}}$ and $m_{\mathrm{L}}=2 \mu_{\mathrm{B}}$. The spin moment for an individual Fe atom is thus twice the value of the corresponding bulk material. On the other hand, the orbital moment for bulk iron is only $m_{\mathrm{L}}=0.086 \mu_{\mathrm{B}}$ per atom and thus more than one order of magnitude smaller than the value of an Fe atom. The orbital moment in clusters is very sensitive to neighbouring atoms and decreases rapidly with an increasing coordination number, only the two outer shells of clusters show values being significantly different from bulk values [34]. In conclusion, the spin moments of surfaces, nanostructures and clusters larger than 100 atoms show a relatively small enhancement, on the other hand, the orbital moment maybe enhanced by a factor of $2-3$.

Deposited magnetic clusters have typically diameters of less than about $5 \mathrm{~nm}$ and are therefore well below the critical dimension at which it becomes energetically favourable to form domain boundaries. As isolated particles they can be considered as single giant moments of ferromagnetically coupled atomic spins that can be aligned by the application of an external magnetic field. When a saturating field is removed the magnetization decays with a relaxation rate. At room temperature the thermal energy is much greater than the anisotropy energy of each particle so that all magnetization directions are almost energetically equal. The magnetization is then described by the classical Langevin function [35] containing the magnetic moment of each cluster. This can be several hundred atomic spins and is unlike isolated atoms where very low temperatures or very high fields are required to achieve saturation, whereas assemblies of clusters can be saturated easily. The temperature at which half the cluster moments 
have relaxed is known as the blocking temperature $T_{\mathrm{B}}$. Additionally, particles on surfaces and in matrices can be accumulated to a sufficient density to study the effects of interactions.

The cluster-surface interactions will modify the free particle magnetic behaviour. The comparison of the ratio $m_{\mathrm{L}} / m_{\mathrm{S}}$ with the bulk values has been performed for mass-filtered Fe clusters deposited onto surfaces (small Fe clusters on graphite [36] and large Fe clusters on cobalt films [37]) as well as for Co islands grown on the herringbone reconstructed $\mathrm{Au}\left(\begin{array}{lll}1 & 1 & 1\end{array}\right)$ surface [38]. Both types of sample exhibit ratios that are greater than the bulk value and increase with decreasing particle size. In Section 5, the data for free Fe clusters will be compared to small mass-selected clusters deposited on HOPG in UHV both exposed to vacuum and capped with metal films. The experimental results showing enhanced magnetic moments in the case of cobalt capping layer are confirmed by theoretical studies of Fe clusters embedded in $\mathrm{Co}$ [39]. The interaction of Fe and Co clusters with a non-magnetic matrix is described in Section 6 for different volume fractions, from isolated particles to volume fractions above the percolation threshold. Experimental results related to self-organized cobalt and iron clusters grown on $\mathrm{Au}\left(\begin{array}{lll}1 & 1 & 1\end{array}\right)$ are discussed in Section 8. Most of the investigations have been carried out using the element-specific technique of XMCD giving access to both contributions to the total magnetic moment, the orbital $m_{\mathrm{L}}$ and spin moment $m_{\mathrm{S}}$ per valence-band hole $n_{\mathrm{h}}$ independently. The technique is briefly described in Section 4.3.1.

Technical applications of small $3 \mathrm{~d}$ metal clusters are generally hindered by the low blocking temperatures of these nanoscaled materials. A promising way to overcome the superparamagnetic limit are alloy clusters consisting of a combination of a $3 \mathrm{~d}$ metal with a high spin moment (Fe, Co) and, e.g. $4 \mathrm{~d}$ or $5 \mathrm{~d}$ metals (such as $\mathrm{Rh}, \mathrm{Pd}, \mathrm{Pt}$ ) which induce a large orbital moment (via the much higher spin-orbit interaction) that stabilizes the magnetization in the clusters. However, the magnetic properties are strongly related to the stoichiometric composition and the crystallographic structure in such alloy clusters. FePt clusters, which have been considered as building blocks for magnetic storage devices [11], have to be present in a special crystallographic phase (the $\mathrm{L} 1_{0}$-phase) in order to show appropriate magnetic properties. This requires a thermal treatment of the clusters at several hundreds ${ }^{\circ} \mathrm{C}$ which leads to strong interdiffusion effects or intermixing with the substrate in case of deposited clusters. First attempts have been made in order to anneal the clusters in the gas phase before deposition [40].

Alloy clusters are not necessarily homogeneous, they may also form core-shell structures as observed for, e.g. cobalt particles with an oxide shell or AgCo nanoparticles where the silver tends to stay at the surface. Such structures may result from the immiscible character of the elements involved, as in the case of $\mathrm{Ag}$ and $\mathrm{Co}$ combined with different physical properties (e.g. strongly different surface energies). Section 7 deals with different types of magnetic alloy clusters, FeCo, CoRh, CoAg, CoPt and CoSm clusters, and focuses on their structural and magnetic properties on surfaces and in matrices.

The review is organized in Sections 2-8, which concern, respectively, the production of clusters and nanostructure formation, the theoretical background, a summary of the magnetic characterization techniques, supported $3 \mathrm{~d}$ transition metal clusters, clusters in matrices, binary alloy clusters, and self-organized clusters on surfaces. Finally, Section 9 points out some general conclusions and perspectives.

\section{Cluster production and nanostructure formation}

This section gives an overview of different techniques for creating clusters and nanostructures on surfaces including their characterization with transmission electron microscopy (TEM), in situ scanning 
tunneling and atomic force microscopy. The first three parts describe methods based on cluster formation, the last part is related to self-organization of clusters on surfaces.

\subsection{Gas aggregation source}

The starting point for gas aggregation is the evaporation of a solid or a liquid material into an aggregation chamber, which is filled with a cold gas at several mbar. Upon reaching supersaturation after many collisions within the inert gas, the atoms nucleate to clusters or particles. The formation of smoke, fog, and clouds in nature occurs according to the same principle. First efficient cluster sources were developed by Mühlbach et al. [41]. Since then many groups use this technique (see [42]).

In the set-up used here, the aggregation cluster source produces pure Fe and Co nanoclusters in the size range 150-700 atoms (or a diameter between 1.5 and $2.5 \mathrm{~nm}$, respectively). It was described previously in [43] and is shown schematically in Fig. 1. It is a UHV gas aggregation source producing a continuous flux in which the metal vapour is generated thermally, using an alumina crucible with embedded tungsten heater, and mixed with He gas cooled to $100 \mathrm{~K}$ at a pressure in the range of 10 mbar. The aggregation region in which the nanoclusters form incorporates aerodynamic lenses described by Tafreshi et al. [44], so that there is an efficient transfer of clusters through the first differential pumping aperture. Mass selection is achieved by ionizing the clusters with a conventional electron bombardment ionizer and passing the ions through a quadrupole mass filter operating at masses up to 350,000 amu (5 $\mathrm{nm} F$ clusters). The mass resolution is approximately $1 \%$ but the resolution/flux trade-off can be altered with a simple electronic adjustment.

Fig. 2 shows in the left part an in situ STM image of unfiltered Fe clusters from the cluster source presented in Fig. 1 deposited on $\operatorname{Si}\left(\begin{array}{lll}1 & 1 & 1\end{array}\right)$ [45]. The dots in Fig. 2b show the diameters measured directly from the STM images of exposed clusters in UHV. The histogram in Fig. $2 b$ was obtained by fitting Langevin functions to the magnetization curves of Fe clusters embedded in Ag with the source operating

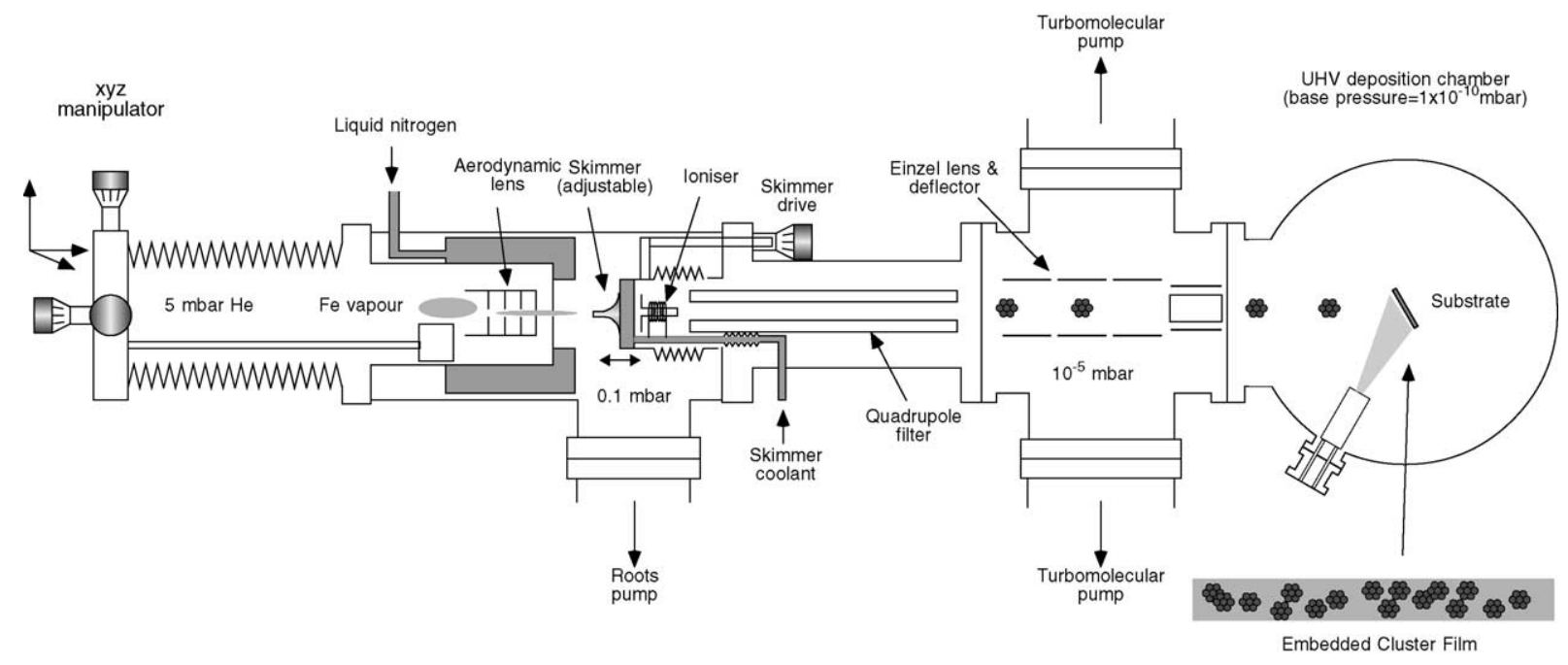

Fig. 1. Schematic of cluster source used to produce pure Fe and Co clusters [43]. It consists of the gas aggregation area (left part), the mass selector (quadrupole filter in the middle) and the UHV deposition chamber (right part). Set-up of the Binns' group, Leicester. 


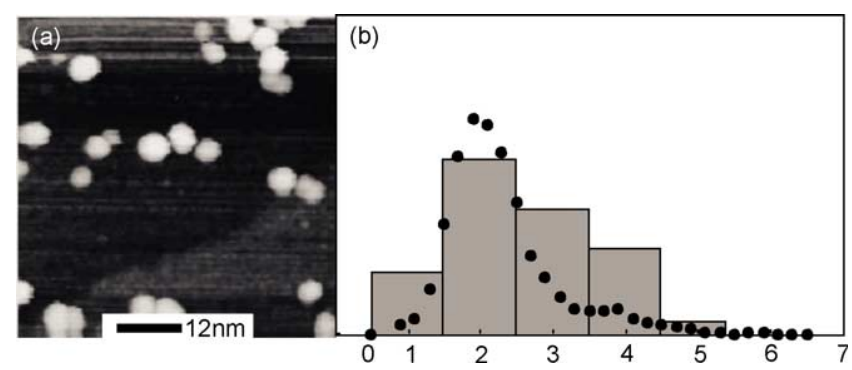

Fig. 2. (a) In situ STM image (40 $\mathrm{nm} \times 40 \mathrm{~nm}$ ) of unfiltered Fe clusters deposited on $\operatorname{Si}\left(\begin{array}{lll}1 & 1 & 1\end{array}\right)$ [46]. (b) Heights (in $\left.\mathrm{nm}\right)$ of Fe clusters on $\operatorname{Si}\left(\begin{array}{lll}1 & 1 & 1\end{array}\right)$ measured directly from the STM images (dots). The histogram shows the size distribution of clusters embedded in Ag obtained by fitting Langevin functions to the magnetization curves [47].

at similar conditions, cf. Section 6.1. The two distributions correspond as does the distribution obtained by measuring the ion current with a quadrupole mass filter [43]. Thus, the clusters deposited on surfaces retain the free cluster size distribution when they land on a surface or if they are embedded in a matrix. The shape of the distribution can be fitted by a log-normal function (see Eq. (1)) with typical $\sigma$ values in the range 1.8-2.0. Embedded cluster films were produced by co-depositing the gas-phase clusters and an atomic vapour of the matrix material (Ag and $\mathrm{Co}$ in this review) onto a common substrate as shown in Fig. 1. Thus, granular films could be produced with independent control over the size and volume fraction and the results presented in Sections 5 and 6 are for cluster films with volume fractions varying from the dilute limit to films of pure clusters with no matrix. Two important aspects of the structure of cluster films, apparent in Fig. 2a, are that the clusters show facets and that neighbouring clusters tend to come into contact along the facets. It is clear from the image that a strong exchange interaction will be invoked between neighbouring clusters. We will demonstrate in Section 6 that the crystalline alignment of the clusters is randomly distributed over three dimensions.

\subsection{Arc cluster ion source}

The continuously working arc cluster ion source (ACIS) has been developed to produce a high flux of mass-filtered metal clusters in the size regime from about 3 to $15 \mathrm{~nm}$. The technique is based on an arc discharge in a hollow cathode, cf. Fig. 3. The complete source is UHV compatible, the target material is

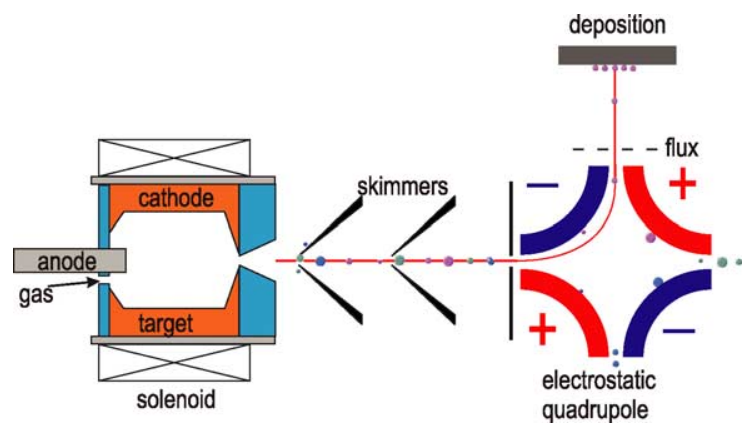

Fig. 3. Schematic view of the continuously working arc cluster ion source. The left part displays the area where the clusters are generated in the hollow cathode made of the target material. The right part shows the mass-filtering unit, which consists of an electrostatic quadrupole deflector [48]. Set-up of the Meiwes-Broer/Bansmann group, Rostock. 
eroded from the cathode. In the cases presented here, the material is usually iron with a purity of more than $99 \%$ or an FeCo alloy. Clusters start to nucleate in the presence of the argon seeding gas and are finally accelerated by a weak supersonic expansion to approximately the velocity of the gas. In order to reduce the high amount of seeding gas, the cluster beam passes through two skimmers. Two oil-free pumping stages are equipped with: (i) a roots pump in combination with a large scroll pump and (ii) a turbomolecular pumping stage. The background pressure is reduced by several orders of magnitude. In order to carry out a mass filtering, charged particle are required that can be deflected in an electric field. Thus, for an efficient deposition, a high portion of the clusters have to be charged. This has been tested by applying an electric field perpendicular to the direction of the beam and monitoring the deflection of the clusters and their intensity in comparison to the neutral part in the beam. The experiment is briefly described in [48]. It has be found that about half of the particles are charged, with equal amounts of positive and negative charges. Assuming a nearly constant velocity $v$ of the incoming clusters due to the supersonic expansion and an equal charge state, it is possible to carry out size filtering in an energy dispersive analyzer known from electron and ion spectroscopy. In the current set-up, a $90^{\circ}$ electrostatic quadrupole deflector is used. After passing the mass-filtering unit, the kinetic energy $E$ of the cluster ions, in our case less than $0.1 \mathrm{eV}$, is clearly below the fragmentation threshold. Details of the cluster source are described by Methling et al. [48]. The amount of cluster material can be estimated either, by measuring the current of the cluster ions upon deposition or at a small mesh at the exit slit of the quadrupole deflector. It should be noted that a base pressure of $1 \times 10^{-10}$ mbar, without cluster beam, is commonly reached in the UHV chamber where the quadrupole deflector is located. A valve between the skimmers and the deflecting unit serves to maintain UHV conditions even if the target has to be replaced.

For the size characterization of metal clusters from the arc cluster ion source, transmission electron microscopy has been performed on nanoparticles deposited onto TEM grids covered with an amorphous carbon layer. The deposition itself takes place under UHV conditions, the sample with the uncapped clusters is subsequently transferred under ambient conditions to the TEM. In this contribution, we will focus on Fe clusters and FeCo alloy clusters. Without mass filtering, the source produces a very high flux of nanoparticles, but with a broad size distribution, cf. Fig. 4. In this case, the deposition has been carried out in forward direction behind the deflector unit. Thus, all kind of particles (charged as well as uncharged) are deposited directly onto the TEM grid. The right part shows the corresponding size distribution starting from a particle size $d$ of about $3 \mathrm{~nm}$ to more than $20 \mathrm{~nm}$ with a maximum close to 8$9 \mathrm{~nm}$. The size of the particles is related to the log-normal distribution (Eq. (1)), typical values for the width of the distribution $\sigma$ are in the range of 2.2:

$$
f(d)=\frac{1}{\sqrt{2 \pi} \sigma d} \exp \left(\frac{\ln d^{2}}{2 \sigma^{2}}\right) .
$$

For charged clusters, the mass-filtering unit shown in Fig. 3 offers the possibility to narrow down the size distribution significantly and to adjust the cluster diameter. In order to vary the size of the clusters between 3 and $15 \mathrm{~nm}$, the voltage of the electrostatic quadrupole deflector has to be tuned between 125 and $5000 \mathrm{~V}$. The effect of mass filtering is demonstrated for Fe clusters in a sequence of figures (cf. Figs. 4 and 5). A detailed evaluation of these TEM results, cf. the histograms in the lower panels of Fig. 5, clearly shows the different size regimes of each sample. The size distributions exhibit sharp lower limits. The limit to larger sizes is probably influenced by multiple charged clusters and the onset of a velocity slip. Both effects lead to an additional contribution of larger clusters. The resolution, e.g. for Fe clusters with a mean size of $12.0 \mathrm{~nm}$ is about $\pm 1 \mathrm{~nm}$. Results on the magnetic properties are described in Section 

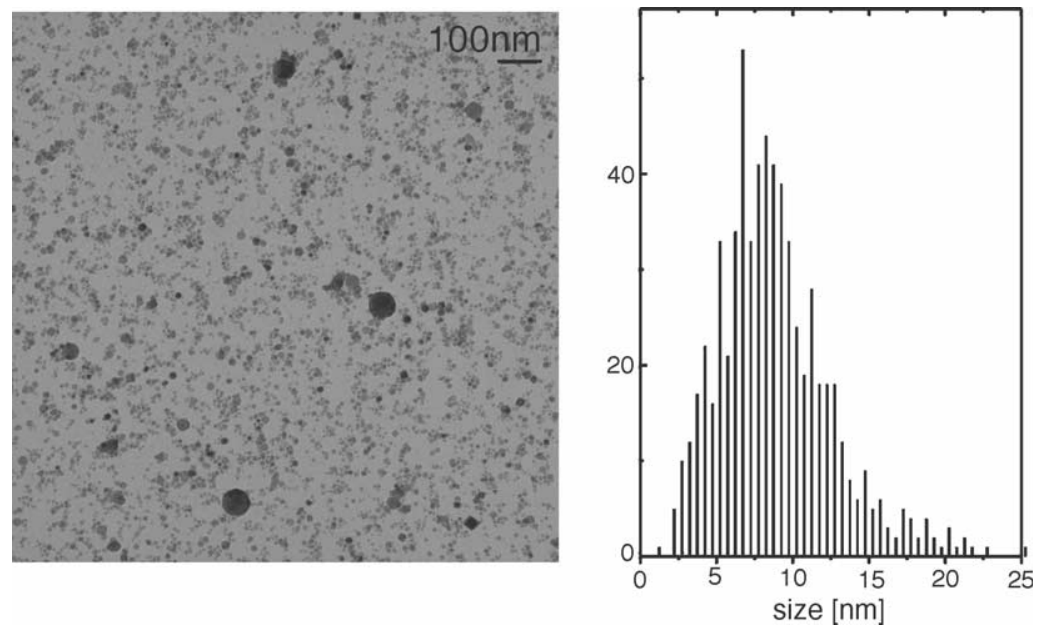

Fig. 4. TEM images and histogram of unfiltered Fe nanoparticles. Note the broad size distribution when compared to the massfiltered deposits results shown in Fig. 5 [49].

5. The ACIS cluster source can also be used to fabricate alloy clusters from a target, the corresponding structural data and magnetic results are shown in Section 7.1.

Furthermore, the chemical composition of all clusters has been characterized by in situ deposition experiments at the storage ring BESSY in Berlin with X-ray absorption spectroscopy (XAS) using tuneable synchrotron radiation. With the UHV standard source, it is possible to perform deposition
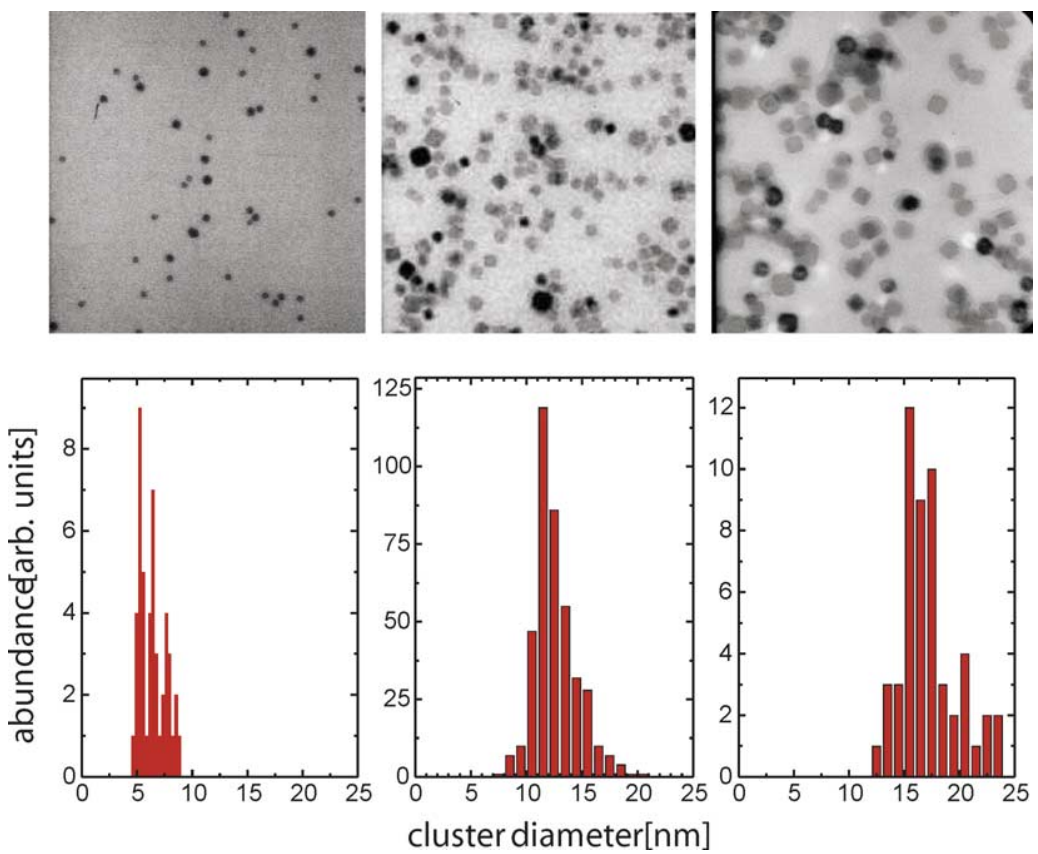

Fig. 5. TEM images (upper panels) and related histograms (lower panels) for mass-filtered Fe clusters with a mean size of $6.1 \mathrm{~nm}$ (left), $12.0 \mathrm{~nm}$ (middle), and $16.4 \mathrm{~nm}$ (right part) [49]. 
experiments being free of contaminations. Even small amounts of contaminations (e.g. oxygen) could be traced in photoabsorption spectroscopy.

\subsection{Laser vaporization cluster source}

Bondybey and English [50] and Andriotis and Menon [51] introduced the concept of laser vaporization (for nearly any solid material) and subsequent cluster growth in a stream of a cold carrier gas. This technique has become a reliable tool to produce intense cold cluster ion and anion beams without any additional ionizing agent (see [52]). In the experiment, cluster assembled films are prepared by deposition of low-energy clusters preformed in the gas phase using such a laser vaporization source (see Fig. 6). Cluster assembled films are prepared by deposition of low-energy clusters preformed in the gas phase using a specially designed equipment schematically represented in Fig. 6. Intense supersonic jets of nanoclusters can be produced with sizes ranging from a few tens to a few thousands of atoms (diameter: 1 to a few nm), typically. Briefly, a Nd:YAG laser $(\lambda=532 \mathrm{~nm}$, pulse duration: a few $\mathrm{ns}$, frequency: $\leq 30 \mathrm{~Hz})$, or a Ti-Sapphire laser $(\lambda=790 \mathrm{~nm}$; pulse duration: a few $\mu \mathrm{s}$; frequency: $\leq 30 \mathrm{~Hz})$ are used to vaporize the target rod mounted in the source chamber (see Fig. 6). For the generation of binary clusters, two target rods can simultaneously be evaporated [53]. Combined with the laser-pulse, a high pressure gas-pulse (He, 3-6 bar; pulse duration: 200-500 $\mu \mathrm{s}$ ) is injected in the source to rapidly cool the plasma generated at the target surface and to nucleate clusters, which are subsequently cooled and stabilized in the supersonic expansion taking place at the exit nozzle of the source.

The main feature of this type of cluster source compared to other ones currently used (thermal, sputtering) is the very high cooling rate up to about $10^{10} \mathrm{~K} / \mathrm{s}$, which governs the formation of original nanoscale systems in non-equilibrium conditions. From the key parameters of the source (laser pulses, gas pulses/continuous gas flow, delay between both pulses, and geometries of the nucleation chamber in the source and the nozzle), it is possible to control the cluster size distributions measured in the highresolution time-of-flight (TOF) mass spectrometer mounted behind the skimmer (Fig. 6). In this case, neutral clusters are photo-ionized using a tuneable excimer-pumped dye laser, then deviated and accelerated at the entrance of the TOF-line. At this stage, photo-ionization near threshold and photo-fragmentation experiments are performed on free clusters to characterize their properties. In the particular case of free $\mathrm{Co}$ and $\mathrm{Ni}$ clusters, icosahedral structures are clearly observed in a size range up to a few thousands of atoms [55] while for Fe clusters the situation seems more complicated probably due to the competition between different structures [55]. For mixed cluster production (i.e. bimetallic), two methods have been developed based on the use of a compound target directly mounted in the source or a special source with two independent targets simultaneously vaporized by two independent lasers as mentioned earlier (Fig. 6). When using the technique with a compound target, it has been verified for various bimetallic systems studied (i.e. Pt-Pd [56], Au-Ni [57], Au-Ag [58], Co-Sm [59]) that the clusters exhibit the same compositions of the target rod. However, depending on some specific effects such as segregation, atoms can be inhomogeneously distributed within the clusters.

After the first stage of free cluster studies in flight, deposition experiments to grow nanostructured films from clusters were performed. In these experiments, only neutral clusters having a very low energy gained in the supersonic expansion at the exit of the source (less than a few $10^{-2}$ eV per atom) are deposited on substrates in the UHV deposition chamber mounted on line with the cluster generator TOF arrangement (Fig. 6). In this case, the complete distribution of neutral clusters (nearly Gaussian in shape and rather narrow when using the laser vaporization generator) are directly deposited on the substrates to 


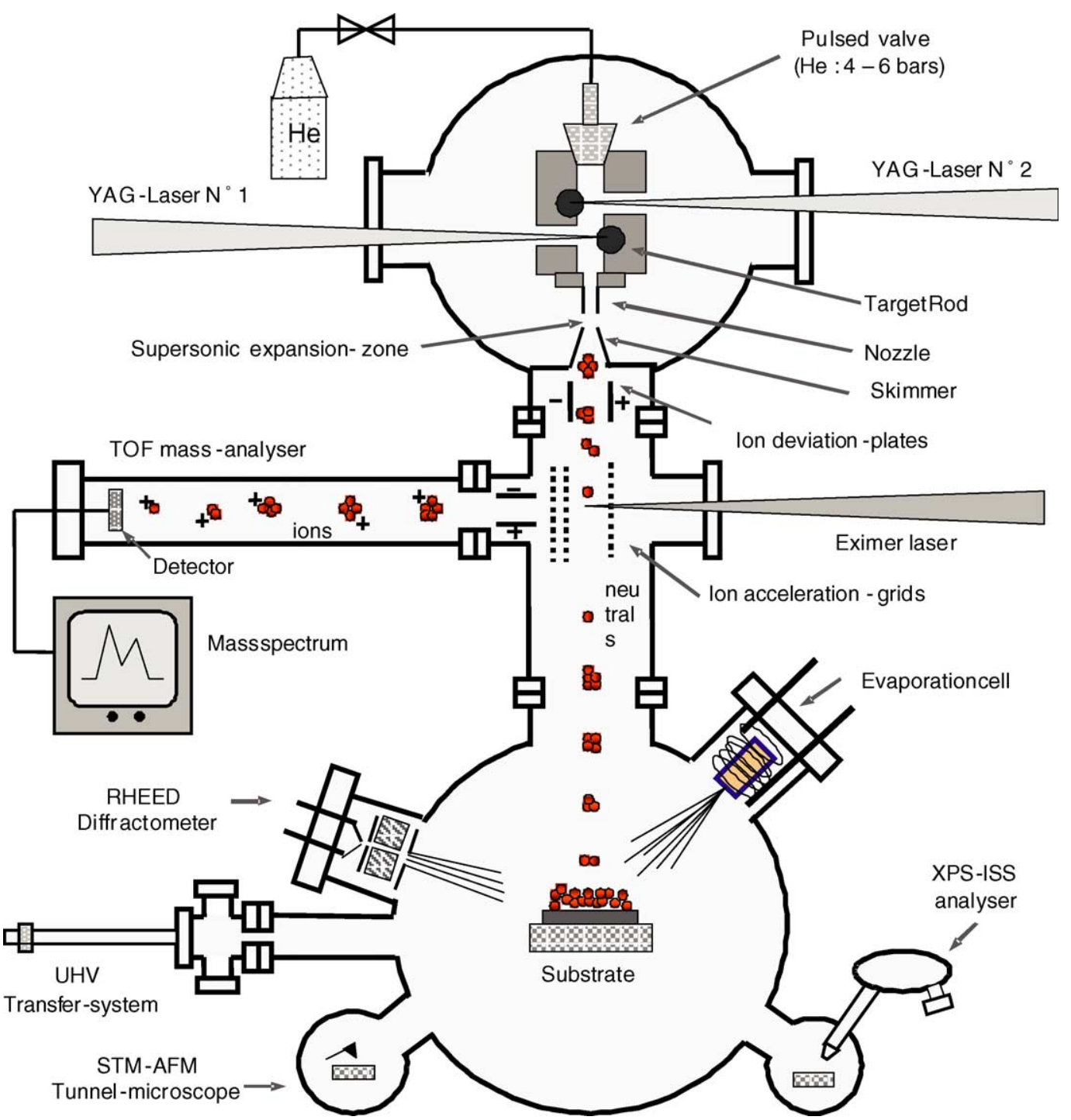

Fig. 6. Schematic view of the cluster generator based on a combined laser vaporization/inert gas condensation source, associated to a time-of-flight mass spectrometer for the studies of free clusters and a UHV deposition chamber for cluster assembled film preparations. The source can operate with one or two independent laser/target arrangements for the production of pure clusters as well as mixed clusters. Some characterization techniques of the films are available in situ in UHV: RHEED diffractometry, STM-AFM microscopy, XPS-ISS spectrometry. An UHV transfer system is used for sample transfers to ex situ equipments. An electron-beam evaporator in situ in UHV is used for co-deposition experiments to produce films of clusters embedded in various media. Set-up of the Perez group, Lyon.

grow films, which allows to reach relatively high deposition rates $(0.1 \mathrm{~nm} / \mathrm{min}$ to $0.1 \mathrm{~nm} / \mathrm{s})$ for all kinds of clusters. Moreover, by co-depositing on the same substrate an atomic beam emitted by the e-beam evaporator mounted in the deposition chamber (cf. Fig. 6), it is possible to produce films of clusters embedded in various matrices (metallic, transparent oxides). In this last case, since both cluster and 
atomic beams are independent, this technique allows the preparation of any kind of cluster/matrix system, even with miscible elements, in a wide range of cluster concentration.

The characteristic nanostructured morphologies of cluster assembled films as a function of the deposition parameters (nature, mean size, and flux of the incident free clusters, nature and temperature of the substrate) are observed in situ using the STM-AFM microscope mounted in the UHV deposition chamber [60] (Fig. 7). Complementary high-resolution transmission electron microscopy observations (HRTEM) are performed ex situ to characterize the atomic structure of isolated supported clusters and cluster assembled nanostructures [61]. In this case, deposits below the 2D percolation threshold (coverage rate: 50\%) are performed on amorphous carbon coated grids and subsequently protected by a thin amorphous carbon layer on top before removing in air. Structural characterizations on collections of clusters by X-rays techniques (diffraction and absorption (EXAFS)) [61,62] are generally performed in addition to HRTEM observations. From both these experimental and theoretical studies it is clearly shown that in the low-energy regime, clusters are not fragmented upon impact on the surface of the substrate. Deposited clusters as large as a few thousands of atoms can diffuse quite fast on the substrate. A key parameter to control this process is the misfit between both lattices at the cluster/ substrate interface, in addition to the substrate temperature [54,63-66]. Up to room temperature, experimental observations as well as molecular dynamics simulations show that the coalescence between two adjacent clusters to form a larger nanostructure is limited leading to a characteristic two dimensional growth process of the cluster film $[33,67]$. This is particularly justified for clusters of moderately high
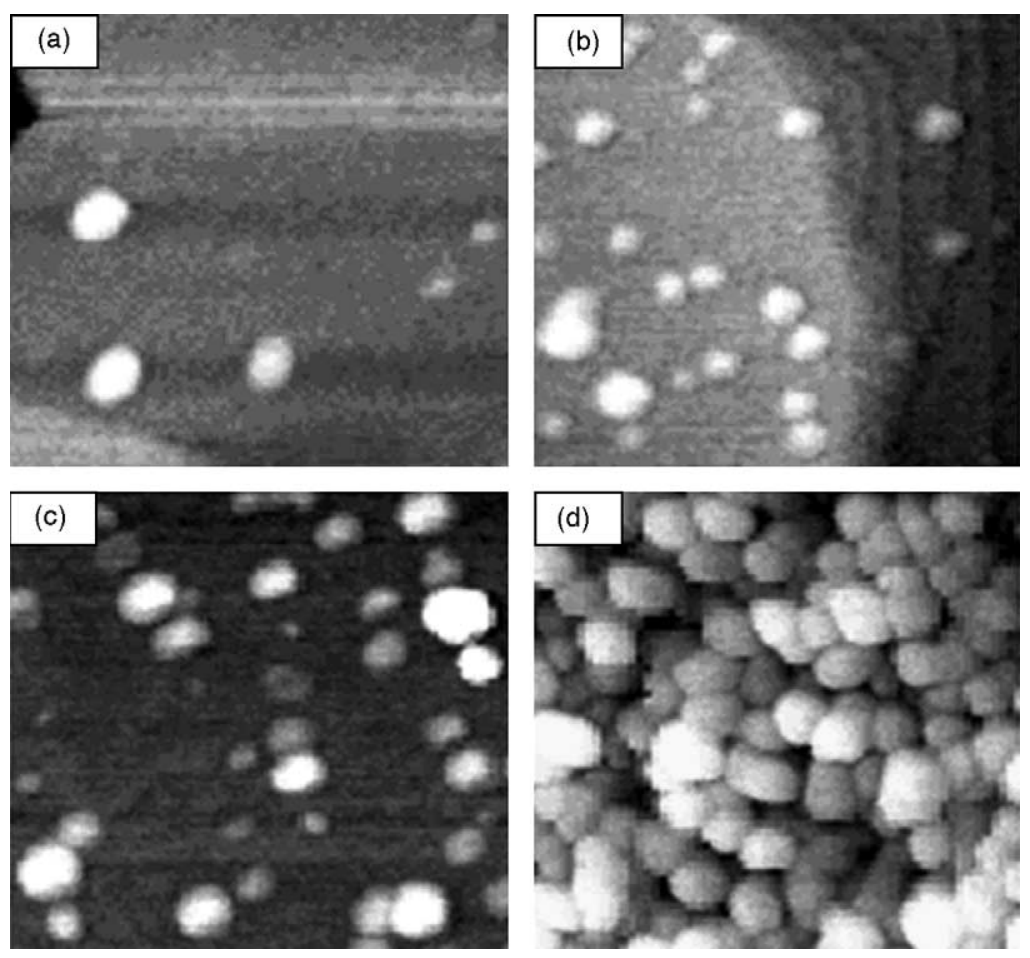

Fig. 7. STM images $(100 \mathrm{~nm} \times 100 \mathrm{~nm})$ of thin $\mathrm{Au}$ films produced by depositing $\mathrm{Au}_{\langle 750\rangle}$ clusters on $\mathrm{Au}(111)$ at room temperature with a flux $F=10^{-3} \mathrm{~nm} / \mathrm{s}$, for different equivalent thicknesses $e_{\mathrm{q}}$ : (a) $e_{\mathrm{q}}=0.01 \mathrm{~nm}$; (b) $e_{\mathrm{q}}=0.03 \mathrm{~nm}$; (c) $e_{\mathrm{q}}=0.08 \mathrm{~nm} ;(\mathrm{d}) e_{\mathrm{q}}=3.4 \mathrm{~nm}[54]$. 

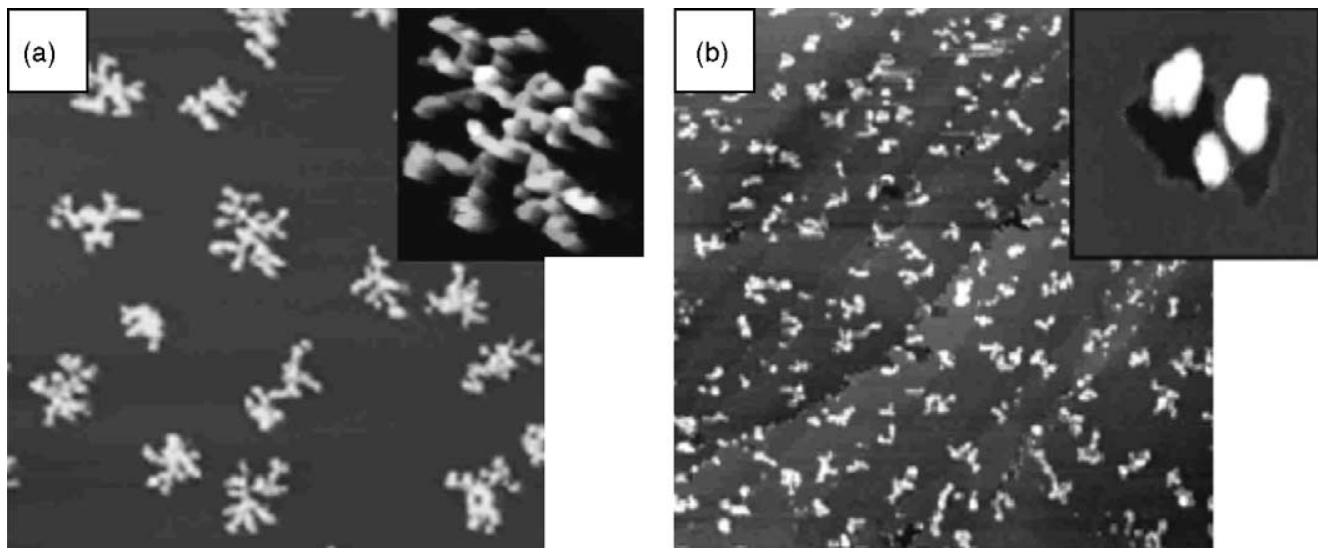

Fig. 8. AFM images $(1000 \mathrm{~nm} \times 1000 \mathrm{~nm})$ of $0.5 \mathrm{~nm}$ thick gold films prepared by $\mathrm{Au}_{750}$ cluster depositions on HOPG graphite substrates at room temperature: (a) virgin substrate; (b) ion-irradiated substrate ( $2 \mathrm{keV} \mathrm{Ar}^{+}$ions). The characteristic ramified island morphologies formed by juxtaposition of deposited clusters is clearly observed in inset of figure a $(100 \mathrm{~nm} \times 100 \mathrm{~nm})$. Note that clusters can easily diffuse on HOPG at room temperature. Different island morphologies are observed in (b) due to the trapping of the diffusing clusters by radiation defects. Three gold clusters trapped at the border of a nano-hole created by $\mathrm{Ar}^{+}-$ion impact are shown in inset of the right figure $(25 \mathrm{~nm} \times 25 \mathrm{~nm})$.

melting temperature materials as in the cases of the magnetic systems under consideration in our experiments. Having control on the above-mentioned cluster/substrate characteristic parameters, specific nanostructure morphologies can be stabilized on the substrate between the two extreme cases: (i) isolated supported clusters randomly distributed on the substrate when the diffusion process is very limited [54] (Fig. 7) and (ii) ramified islands formed by cluster aggregation when diffusion is taking place $[54,66]$ (Fig. 8a). In this case, various defects on the surface of the substrate act as traps for the diffusing clusters leading to the creation of nucleation-centres for the formation of cluster islands.

Surface-defects to trap clusters can be natural (i.e. atomic steps, etc.) or artificially created. This is well illustrated in Fig. 8b, showing the morphology of a cluster film deposited on an ion-irradiated HOPG substrate compared to the morphology of the film deposited in the same conditions on the non-irradiated HOPG substrate (Fig. 8a). Randomly distributed defects in the form of nano-holes resulting from energetic heavy-ion impacts are the cause of the trapping process of the diffusing clusters as shown in the inset in (Fig. 8b). The capability of surface defects to influence the nucleation and growth process of cluster assembled films could be efficiently used in the near future to produce 2D-organized arrays of nanoclusters on functionalized substrates for applications in the areas of high density magnetic-memory devices, spin electronics, and new, novel quantum devices. In this context, preliminary experiments to deposit clusters on HOPG substrates exhibiting a 2D-organized array of nanosize defects obtained by the FIB-nanoengraving technique have been carried out [68] (Fig. 9a-c). Clusters concentrated around the nano-defects, as shown in Fig. $9 \mathrm{~b}$ and c, show promise as precursers for future experiments to build wellordered 2D arrays.

\subsection{Self-organization of clusters formed on reconstructed surfaces}

When atoms are adsorbed on a flat single-crystal surface, they move and stick together to form aggregates. At first sight one would expect that if atoms diffuse in chaotic random motion, what they 

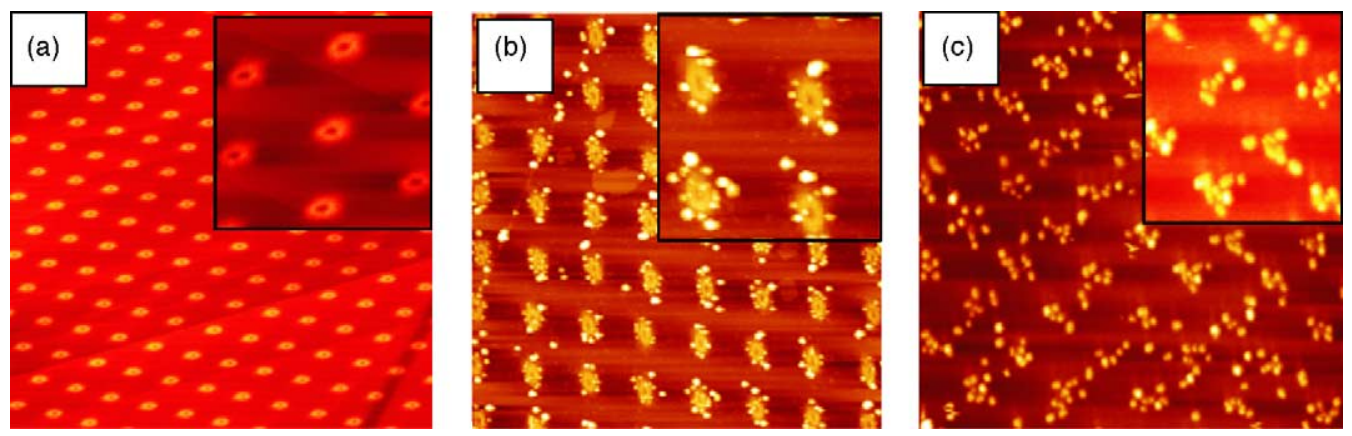

Fig. 9. AFM images of a FIB-structured HOPG substrate $(3.4 \mu \mathrm{m} \times 3.4 \mu \mathrm{m})(\mathrm{a})$ and $2 \mathrm{D}$-organized arrays of clusters $(2 \mu \mathrm{m} \times$ $2 \mu \mathrm{m}$ ) obtained by deposition of $\mathrm{Au}_{750}$ clusters $\left(10^{-2} \mathrm{ML}\right)$ on FIB-functionalized HOPG substrates at $373 \mathrm{~K}$ (b and c). Using the FIB-nanoengraving technique with $30 \mathrm{keV} \mathrm{Ga}^{+}$ions, depending on the ion-fluence per point, various types of 2D-organized arrays of nanosize defects are created on HOPG substrates (i.e. array of nanosize holes with a lattice parameter of $300 \mathrm{~nm}$ as shown in inset of image (a) with a size of $500 \mathrm{~nm} \times 500 \mathrm{~nm}$ ). Gold clusters subsequently deposited on these substrates are trapped on the defects (see insets $(500 \mathrm{~nm} \times 500 \mathrm{~nm}$ ) of images (b) and (c)) to form organized arrays. In the case of image (c), the FIB fluence per point was 10 times lower than in the cases of images (a) and (b). For such a low FIB-fluence, the defects consist on nanosize bumps, which seem quite efficient to trap the diffusing gold clusters as observed in inset of image (c). The preparation of magnetic cluster arrays is in progress.

build must look like shapeless debris. One of the most fascinating discoveries in surface physics during the last decade was that adsorbed atoms may lead to well organized aggregation patterns whose structure can be manipulated experimentally (for a review, see [3]). In other words, the density, shape, distribution of clusters (or any small entity of atoms) can be controlled to a large extent without any external intervention by controlling the physical parameters such as substrate temperature, substrate crystallographic symmetry and deposition rate. In this context, the controlled formation of ordered metal nanostructures on solid surfaces by self-organized growth, allows one to anticipate new data storage technologies based on nanoscale dots with tuneable densities in excess of 1 Tbits/in. ${ }^{2}$.

A possible scenario of island formation and growth is as follows: when an incoming adatom is adsorbed on the surface from the gas phase it may have two distinct destinies: (i) it may meet another adatom during its thermally activated random walk and form a stable nucleus (an immobile dimer), or (ii) it may be directly incorporated into an already existing island. The later step does not contribute to increase the number of islands on the surface, it just increases the size of an existing island. The first event is much more frequent in the first stage of the growth process while the incorporation into an already existing island will dominate at longer times. At very long time, no new islands are formed and an incoming adatom diffuses to an existing island before it has the opportunity to meet another adatom. As a result, the island density saturates and we can define a capture area for each island. The average separation between islands then provides a good measure of the adatom diffusion length $\Lambda_{\mathrm{a}}$. An analytical expression of this saturation density of islands $N_{\text {sat }}$ has been proposed by Venables et al. [69]:

$$
N_{\text {sat }}=\eta(\theta)\left(\frac{R}{v_{0}}\right)^{i /(i+2)} \exp \left(\frac{E_{i}+i E_{\mathrm{d}}}{k_{\mathrm{B}} T(i+2)}\right),
$$

where $\eta$ is a slow varying function of the coverage $\theta, R$ is the deposition rate and $v_{0}$ is the attempt frequency of the order of $10^{13-1014} \mathrm{~Hz} . E_{\mathrm{d}}$ is the energy barrier for adatom diffusion on the surface. The size of the critical nucleus is $i$ and $E_{i}$ is its binding energy. $(i+1)$ is the smallest island still stable at a 
given temperature. When the critical nucleus is one, $E_{i}$ becomes zero and we get the exponent $E_{\mathrm{d}} / 3$. The formula reflects the fact that the density of islands can be adjusted since it depends exponentially on temperature and on parameters such as the energy barrier $E_{\mathrm{d}}$. On a homogeneous surface, isotropic clusters will be distributed randomly. Under certain circumstances however (anisotropic diffusion or anisotropic bonding) atomic chains and stripes can be grown [70,71]. In this case, particular symmetries of the surface may play a leading role. For example, by favouring the easy diffusion of adatoms along potential wells or grooves. The anisotropic diffusion can be exploited to tailor highly elongated metastable islands. The simplest example of an anisotropic substrate is a $\left(\begin{array}{lll}1 & 1 & 0\end{array}\right)$ surface of a fcc crystal. Such a substrate is made of compact atomic rows, along the [ $\left[\begin{array}{lll}1 & \overline{1} & 0\end{array}\right]$ direction, separated by channels. In a favourable case, the adatoms will diffuse preferentially within the channels in a linear type of random motion. The linear chains form spontaneously by aggregation of adatoms diffusing along the channels.

The following part deals with surface reconstruction and strain relaxation patterns owing to their central role as atomic scale templates for the self-organization of small entities at the nanometer scale. Surfaces may reconstruct spontaneously as a result of free energy minimization. This means that with respect to their positions in the bulk crystal, surface atoms adopt new equilibrium positions. An example of such a reconstruction is the chevron reconstruction of the $\mathrm{Au}\left(\begin{array}{lll}1 & 1 & 1\end{array}\right)$ surface [72,73]. It produces rearrangements of surface atoms at the nanoscopic scale that may appear as a modulation of the topmost atomic layer. Dislocation networks that are interesting for self-organized growth are most easily induced by hetero-epitaxy where the lattice mismatch between two different materials is exploited, see the case of the dislocation pattern formed by Ag bilayers on $\operatorname{Pt}\left(\begin{array}{lll}1 & 1 & 1\end{array}\right)$ [74]. The left part of Fig. 10 shows a hard sphere model of a dislocation network on a fcc(lllll 111$)$ crystal, where adjacent domains correspond to different stackings of the topmost layer ( $\mathrm{fcc}$ of hcp). The right parts of Fig. 10 show the example of $\mathrm{Au}\left(\begin{array}{lll}1 & 1 & 1\end{array}\right)$ zigzag reconstruction where the bright contrasts result from the domain walls corrugations visible in the STM images. These discommensuration walls are limiting fcc and hcp stacking domains of the topmost atomic layer, which form spontaneously by strain relaxation. The registry of surface gold atoms varies from hollow sites of the fcc to the hollow sites of the hcp stacking (the fcc regions are wider than the hcp regions). The fcc to hcp transition (the discommensuration wall) appears as ridges in the STM images since surface atoms near bridge sites rest about $0.2 \AA$ higher than in hollow sites.
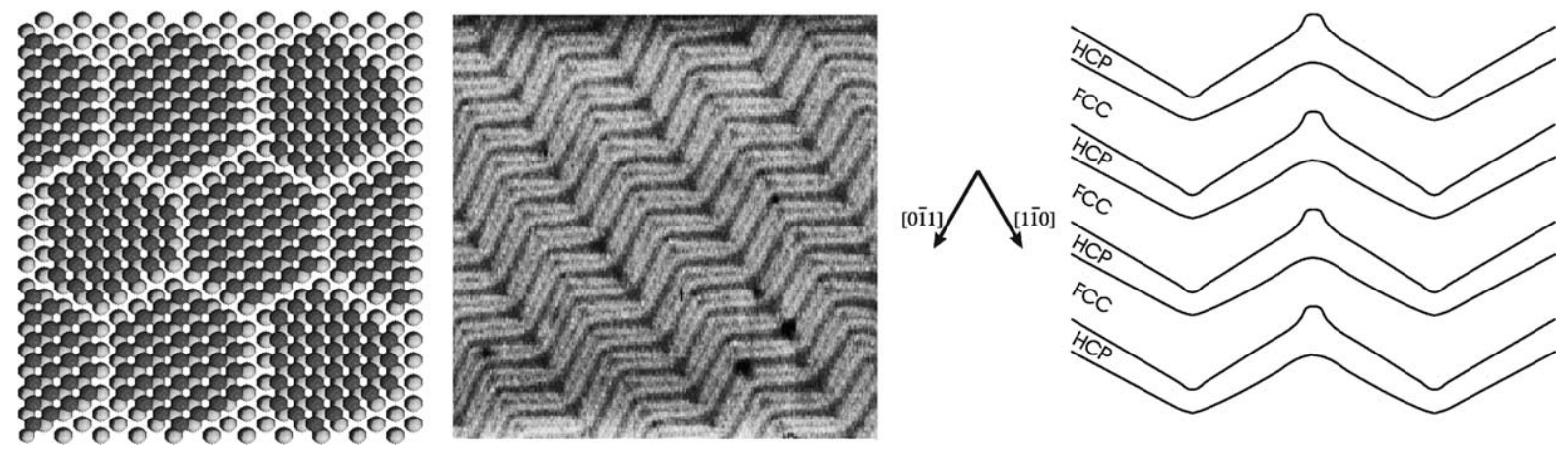

Fig. 10. Left part: schematic diagram of the two-dimensional hard sphere model of fcc and hcp commensurate domains separated by domain walls. Right parts: zigzag reconstruction of the Au(l 11 1) surface with STM image $100 \mathrm{~nm} \times 100 \mathrm{~nm}$ (middle) and schematic view of the domains. 
Due to its particular conformation, the zigzag reconstruction of $\mathrm{Au}\left(\begin{array}{lll}1 & 1 & 1\end{array}\right)$ leads to singularities that can best be illustrated as single atomic site dislocations (five nearest-neighbours instead of six) located at the elbows of the chevron reconstruction [75]. The dislocations are distributed on a rectangular lattice with a unit cell $75 \AA \times 140 \AA$. Although this reconstruction has first been evidenced experimentally, it is fairly well understood today thanks to molecular dynamics simulations [76]. Among other potentially interesting systems with stress relaxation patterns, lets mention $\mathrm{Ag}$ on $\mathrm{Cu}\left(\begin{array}{lll}1 & 1 & 1\end{array}\right)$ [77], $\mathrm{Cr}$ on $\mathrm{Pt}\left(\begin{array}{lll}1 & 1 & 1\end{array}\right)$ [78] and Au on Ni(lll 11 1 $)$ [79], which have been investigated both from the experimental and theoretical point of view.

It has been observed that the $\mathrm{Au}\left(\begin{array}{lll}1 & 1 & 1\end{array}\right)$ surface may be used as a template to grow organized metal clusters of transition metals [75,80-82]. The point dislocations at the elbows of the chevron reconstruction (see Fig. 10) act as nucleation sites for adatoms adsorbed from the gas phase. The adatoms diffuse randomly until finally they reach the point dislocations. The density of islands is then dictated by the density of point dislocations rather than by Eq. (2) for the kinetic growth. Variable temperature STM is used to follow the growth on self-organized templates [83]. Fig. 11 shows an example of self-organized cobalt bilayer clusters containing 300 atoms each. If one would be able to store information in these dots, one would reach a storage density of $10^{12} \mathrm{bits} / \mathrm{cm}^{2}$, namely $10^{3}$ times higher than the highest storage densities reached today. These clusters are stable in UHV environment up to $400 \mathrm{~K}$. Above this temperature, the clusters may burrow into the gold substrate, by simultaneously expelling gold atoms. This is due to the low surface energy of gold, which tends to encapsulate the cobalt clusters. Annealing below $600 \mathrm{~K}$, does not perturb significantly the cobalt clusters since the magnetic properties are preserved. For ex situ measurements, and potential applications, Co clusters and films can easily be stabilized by a protective film of a few gold monolayers.

If the growth temperature is lowered below a given limit, the mean free path of a diffusing adatom may not be enough to reach the point dislocation, and the growth will be dictated by the random nucleation according to Eq. (2). Therefore, low temperature deposition favours growth of small islands with a high

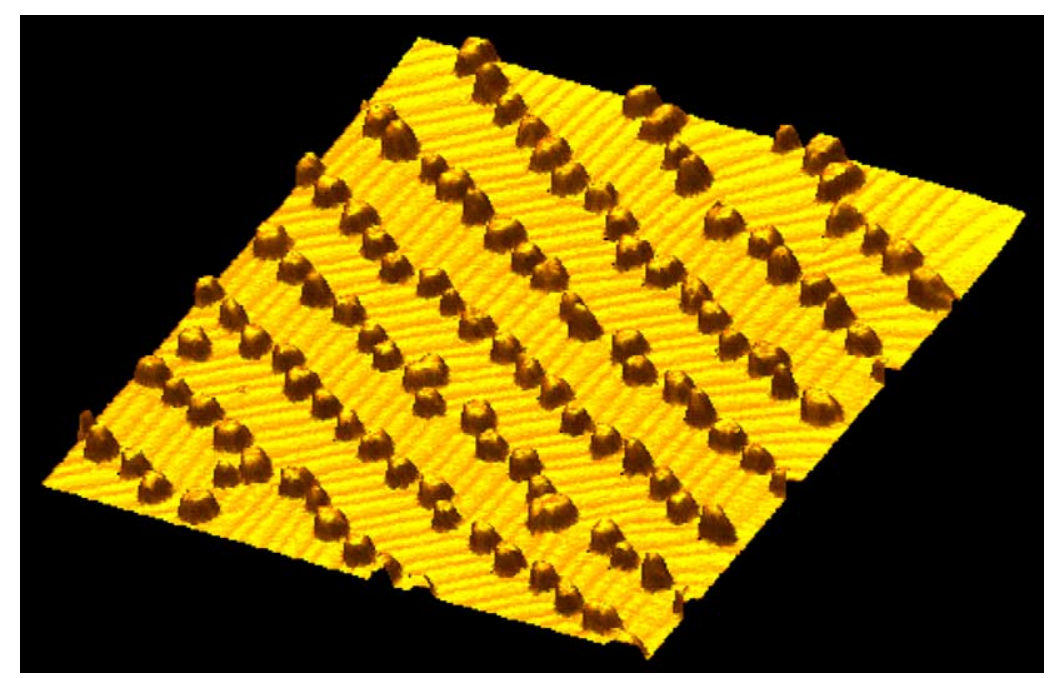

Fig. 11. Cobalt clusters made of 2 Co monolayers, approximately 300 atoms each, self-assembled on the zigzag reconstruction of the $\mathrm{Au}\left(\begin{array}{ll}1 & 1\end{array}\right)$ surface. Image: $100 \mathrm{~nm} \times 100 \mathrm{~nm}$ [83]. 
surface density [84]. This behaviour manifests itself in a quite spectacular way in $\mathrm{Co} / \mathrm{Au}\left(\begin{array}{lll}1 & 1 & 1\end{array}\right)$ because of the large incidence on magnetism. The two different outcomes will be analyzed more in detail in Section 8. Variable temperature STM experiments are shown in Fig. 55 in Section 8.3.

\section{Theoretical background on cluster magnetism}

The purpose of this section is to introduce several basic concepts in the microscopic theory of cluster magnetism with focus on the properties of TM systems [85]. The electronic models relevant for describing the magnetic behaviour of nanometer-scale particles are briefly recalled. Mathematical equations are provided which relate the main properties of experimental and technological interest, such as the spin and orbital magnetic moments per atom, magnetic order, and magnetic anisotropy energy (MAE) with the magnitudes that characterize the cluster electronic structure (e.g. Coulomb and spinorbit interactions, local electronic densities of states, spin-density distribution, etc.). The size and structural dependence of various cluster magnetic properties is analyzed and the microscopic origin of various experimental observations on clusters is discussed.

\subsection{Model Hamiltonians}

We consider the non-relativistic Schrödinger equation for the valence electrons and expand the field operator $\hat{\psi}_{\sigma}(r)=\sum_{i \alpha} \phi_{i \alpha}(r) \hat{c}_{i \alpha \sigma}$ for spin $\sigma$ in a set of orbitals $\phi_{i \alpha}(r)$ centered at each atom $i$, where $\alpha$ refers to the different $\mathrm{s}, \mathrm{p}$, and d orbitals. The Hamiltonian can be written as $H=\hat{H}_{0}+\hat{V}$, where

$$
H_{0}=\sum_{i \alpha, j \beta} t_{i j}^{\alpha \beta} \hat{c}_{i \alpha \sigma}^{\dagger} \hat{c}_{j \beta \sigma}
$$

takes into account the single-particle hybridizations and

$$
\hat{V}=\frac{1}{2} \sum_{\sigma \sigma^{\prime}} \sum_{i j k l} \sum_{\alpha \beta \gamma \delta} V_{i j k l}^{\alpha \beta \gamma \delta} \hat{c}_{i \alpha \sigma}^{\dagger} \hat{c}_{j \beta \sigma^{\prime}}^{\dagger} \hat{c}_{k \gamma \sigma^{\prime}} \hat{c}_{l \delta \sigma}
$$

is the electron-electron interaction. The transfer or hopping integrals $t_{i j}^{\alpha \beta}$ correspond to single-electron transitions between the orbital $\beta$ at atom $j$ and the orbital $\alpha$ at atom $i . \varepsilon_{i \alpha}^{0}=t_{i i}^{\alpha \alpha}$ stands for the singleparticle energy level of the orbital $i \alpha$. The Coulomb integrals $V_{i j k l}^{\alpha \beta \gamma \delta}$ represent transitions of a pair of electrons from at the orbitals $k \gamma$ and $l \delta$ to the orbitals $i \alpha$ and $j \beta$.

The intra-atomic terms in $\hat{V}$ are the dominant ones for the magnetic properties. As already discussed, they are responsible for the Hund-rule correlations that lead to the formation of local magnetic moments in an open $d$ shell. Therefore, in first approximation, we may treat explicitly only the intra-atomic Coulomb terms $(i=j=k=l$ ) by including the inter-atomic Coulomb contributions as a mean-field correction to the single-particle potential and hopping integrals. An explicit treatment of inter-atomic contributions becomes important for heterogeneous clusters, such as TM oxides, where significant sitedependent charge transfers occur.

The Coulomb integrals $V_{i j k l}^{\alpha \beta \gamma \delta}$ are approximately proportional to the product of the overlap between $\phi_{i \alpha}$ and $\phi_{l \delta}$, and between $\phi_{j \beta}$ and $\phi_{k \gamma}$. Thus, the interaction Hamiltonian may be simplified by retaining explicitly only the terms involving at most two different orbitals, namely, the direct contributions having 
$i \alpha=l \delta$ and $j \beta=k \gamma$, and the exchange contributions having $i \alpha=k \gamma$ and $j \beta=l \delta$ (two-center approximation). Thus, the interaction term takes the form:

$$
\hat{H}_{\mathrm{C}}=\frac{1}{2} \sum_{i \alpha \beta \sigma}\left[U_{\alpha \beta} \hat{n}_{i \alpha \sigma} \hat{n}_{i \beta \bar{\sigma}}+\left(U_{\alpha \beta}-J_{\alpha \beta}\right) \hat{n}_{i \alpha \sigma} \hat{n}_{i \beta \sigma}\right]-\frac{1}{2} \sum_{i, \alpha \neq \beta} J_{\alpha \beta}\left(\hat{s}_{i \alpha}^{+} \hat{s}_{i \beta}^{-}+\hat{s}_{i \alpha}^{-} \hat{s}_{i \beta}^{+}\right),
$$

where $\hat{n}_{i \alpha \sigma}$ is the number operator and $\hat{s}_{i \alpha}^{+}=\hat{c}_{i \alpha \uparrow}^{\dagger} \hat{c}_{i \alpha \downarrow}$ the spin-1/2 raising operator $\left[\hat{s}_{i \alpha}^{-}=\left(\hat{s}_{i \alpha}^{+}\right)^{\dagger}\right] \cdot U_{\alpha \beta}=$ $V_{i i i i}^{\alpha \beta \beta \alpha}$ and $J_{\alpha \beta}=V_{i i i i}^{\alpha \beta \beta}$ refer, respectively, to the direct and exchange Coulomb integrals between the orbitals $\alpha$ and $\beta$ of atom $i$. For homogeneous clusters, they are independent of $i . U_{\alpha \beta}$ acts irrespectively of the electrons' spin and controls charge transfers among the different orbitals. $J_{\alpha \beta}$ is responsible for Hund's first rule in the atom and for the formation of spin moments. Finally, the orbital dependence of $U_{\alpha \beta}$ and $J_{\alpha \beta}$ tends to stabilize the formation of orbital moments and leads to Hund's second rule in atoms. Notice that in Eq. (5) we have neglected for simplicity the off-diagonal (scattering) terms of the Coulomb interaction that involve more than two different orbitals. Therefore, $\hat{H}_{C}$ satisfies atomic rotational invariance strictly only when $U_{\alpha \beta}=U$ and $J_{\alpha \beta}=J$ are independent of the orbital quantum number $m$. The calculations of MAE presented in Section 3.4 fulfil this condition. A detailed discussion of results obtained using more general orbital-dependent models is beyond the scope of this review $[85,86]$. With the present computer facilities it is possible to perform mean-field ground-state calculations on small clusters using complex multiband models that treat all $\mathrm{s}, \mathrm{p}$ and $\mathrm{d}$ valence electrons explicitly (e.g. Hartree-Fock or density functional theory in the local spin density approximation). The situation changes completely when electron correlation effects or finite-temperature properties are explicitly addressed. In this case, it is necessary to simplify the valence-electron dynamics further by focusing on the $\mathrm{d}$ orbitals responsible for magnetism. Comparative studies between spd and d models at $T=0$ show, as expected, that the magnetic properties of TM systems are largely dominated by the $\mathrm{d}$ valence electrons. Therefore, one often restricts $\alpha$ and $\beta$ in Eqs. (3) and (5) to the 3d states.

In the mean-field approximation the fluctuations of the correlation term are neglected and the Hamiltonian is given by:

$$
H=\sum_{i \alpha \sigma} \varepsilon_{i \alpha \sigma} \hat{n}_{i \alpha \sigma}+\sum_{i \neq j, \alpha \beta \sigma} t_{i j}^{\alpha \beta} \hat{c}_{i \alpha \sigma}^{\dagger} \hat{c}_{j \beta \sigma},
$$

with

$$
\varepsilon_{i \alpha \sigma}=\varepsilon_{i \alpha}^{0}+\sum_{\beta}\left(U_{\alpha \beta}-\frac{1}{2} J_{\alpha \beta}\right) v_{i \beta}-\frac{\sigma}{2} \sum_{\beta} J_{\alpha \beta} \mu_{i \beta} .
$$

Here, $v_{i \beta}=\left\langle\hat{n}_{i \beta \uparrow}\right\rangle+\left\langle\hat{n}_{i \beta \downarrow}\right\rangle$ and $\mu_{i \beta}=\left\langle\hat{n}_{i \beta \uparrow}\right\rangle-\left\langle\hat{n}_{i \beta \downarrow}\right\rangle$ refer, respectively, to the average occupation and spin polarization of orbital $i \beta(\sigma= \pm 1)$. Notice that we have assumed here for simplicity that the magnetic moments are collinear (i.e. $\left\langle s_{i \alpha}^{+}\right\rangle=0, \forall i \alpha$ ). The collinear state is always a self-consistent solution since, in the absence of spin-orbit interactions, the eigenstates of $H$ are products of spin-up and spin-down states when $\left\langle s_{i \alpha}^{+}\right\rangle=0$. However, it is sometimes possible to find lower-energy solutions having non-collinear arrangements of the local spin polarizations. This is the case when magnetic frustrations are present, for example, in antiferromagnetic compact clusters (see [87-91]).

The average occupation numbers $\left\langle\hat{n}_{i \alpha \sigma}\right\rangle$ are determined self-consistently from:

$$
\left\langle\hat{n}_{i \alpha \sigma}\right\rangle=\int_{-\infty}^{\varepsilon_{\mathrm{F}}} \mathcal{N}_{i \alpha \sigma}(\varepsilon) \mathrm{d} \varepsilon,
$$


where

$$
\mathcal{N}_{i \alpha \sigma}(\varepsilon)=-\frac{1}{\pi} \operatorname{Im}\left\{G_{i \alpha \sigma, i \alpha \sigma}(\varepsilon)\right\}
$$

is the local density of states (LDOS) per spin orbital and $G_{i \alpha \sigma, i \alpha \sigma}(\varepsilon)$ the local Green's function $\left[\hat{G}=(\varepsilon-\mathcal{H})^{-1}\right]$. A particularly efficient way of determining $\mathcal{N}_{i \alpha \sigma}(\varepsilon)$ in systems lacking translational symmetry (free and deposited clusters, surfaces, thin films, etc.) is the Haydock-Heine-Kelly's recursion method [92], which expresses $G_{i \alpha \sigma, i \alpha \sigma}(\varepsilon)$ as a local expansion around each atom $i$ of the cluster. This local approach is physically very appealing since it stresses the role of the local environment as a function of size and composition, or as we move from the interior to the surface of the cluster.

\subsection{Local atomic environment and spin magnetic moments}

The lowest-order local approximation to $\mathcal{N}_{i \alpha \sigma}(\varepsilon)$ is the second-order approximation which is obtained by taking into account only the contributions to $G_{i \alpha \sigma, i \alpha \sigma}(\varepsilon)$ from the nearest-neighbours of atom $i$. Dropping the orbital dependence $\left(U_{\alpha \beta}=U, J_{\alpha \beta}=J\right)$ and averaging over $\alpha$ one obtains:

$$
\mathcal{N}_{i \sigma}^{(2)}(\varepsilon)=\frac{10}{\pi w_{i}}\left\{1-\frac{\left(\varepsilon-\varepsilon_{i \sigma}\right)^{2}}{w_{i}^{2}}\right\}^{1 / 2},
$$

where $w_{i}^{2}=\left(z_{i} / z_{\mathrm{b}}\right) w_{\mathrm{b}}^{2}$ is the second moment of the local DOS. $w_{i}=\sqrt{z_{i} / z_{\mathrm{b}}} w_{\mathrm{b}}$ plays the role of an effective local band-width that depends on the local coordination number $z_{i}$ at the cluster atom $i$. $z_{\mathrm{b}}$ refers to the coordination number in the bulk $\left(z_{\mathrm{b}}=12\right.$ for an FCC lattice, for example) and $w_{\mathrm{b}}$ to the bulk bandwidth.

As illustrated in Fig. 12, the reduction of local coordination number results in a reduction of the local effective band-width and thus in an enhanced paramagnetic density of states $\mathcal{N}_{i 0}\left(\varepsilon_{\mathrm{F}}\right)$ at the Fermi energy. The resulting self-consistent magnetic moments are given in Fig. 13. This very simple approximation already explains qualitatively various major trends in low-dimensional TM magnetism:

(i) The local spin magnetic moments $\mu_{i}$ increase as the local coordination number decreases since the kinetic (promotion) energy involved in the formation of local moments is smaller for larger $\mathcal{N}_{i \sigma}(\varepsilon)$ (Stoner's criterion). Thus, surface atoms have larger spin-moments that the atoms inside the cluster. In addition, the enhancement of $\left\langle S_{i z}\right\rangle$ is more important at open surfaces than at closed ones [93].

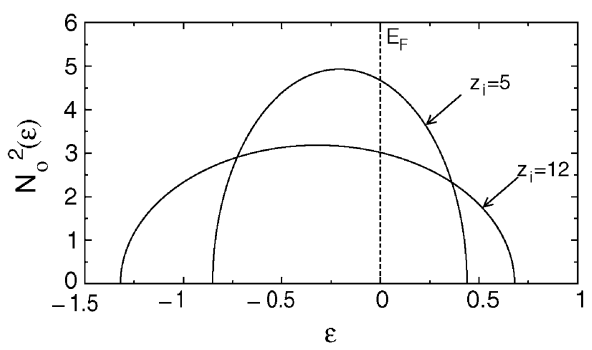

Fig. 12. Local density of states $\mathcal{N}_{i 0}^{(2)}(\varepsilon)$ in the second-moment approximation for the local coordination numbers $z_{\mathrm{i}}=12$ and $z_{i}=5$ (Eq. (8)). The Fermi energy $\varepsilon_{\mathrm{F}}=0$ corresponds to Fe $\left(n_{\mathrm{d}}=7\right)$ [85]. 


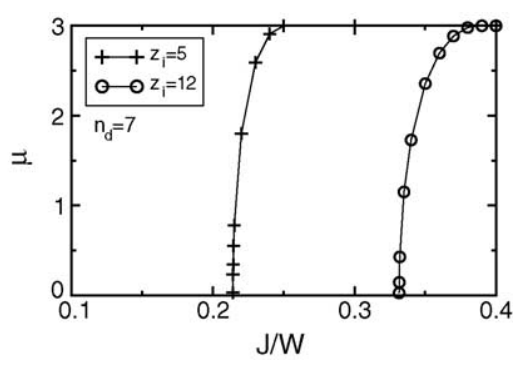

Fig. 13. Self-consistent magnetic moments as a function of $J / w_{\mathrm{b}}$ as obtained by using the second-moment approximation to the local density of states [85], for the local coordination numbers $z_{i}=5$ and $z_{i}=12$.

(ii) The average spin moment per atom $\bar{\mu}_{N}$ of an $N$-atom cluster increases with decreasing $N$ since $\left\langle z_{i}\right\rangle$ decreases. Moreover, the enhancement of $\bar{\mu}_{N}$ originates at the cluster surface where $z_{i}$ is smallest.

(iii) Bond length contraction results in a reduction of $\mu_{i}$ and $\bar{\mu}_{N}$ since for shorter inter-atomic distances the hopping integrals and thus $w_{\mathrm{b}}$ increase.

(iv) It is in principle possible that TMs which are non-magnetic in the bulk could develop non-vanishing magnetizations if the system dimensions are reduced. This has been observed experimentally in the case of $\mathrm{Rh}_{N}$ clusters [24,25]. For specific quantitative calculations on $\mathrm{Rh}_{N}$, see [94-99].

Of course, such a simple structureless LDOS cannot explain many other important properties. Eq. (8) fails to reproduce that in clusters $\bar{\mu}_{N}$ can only adopt discrete values due to the discreteness of the electronic spectrum in a finite system. The same holds for the changes in the local spin moments observed even for atoms having a complete nearest-neighbour (NN) shell below the cluster surface. Eq. (8) is also unable to distinguish between different magnetic orders which may depend on the cluster structure, as obtained, for example, in bcc- and fcc-like Fe clusters [18]. These are just a few examples. Nevertheless, despite these drawbacks, the second moment approximation remains an illustrative start point for a more rigorous analysis of cluster magnetic properties from a local perspective. A detailed review of theoretical studies on clusters may be found, for instance, in [85] and references therein.

To close the discussion at the non-relativistic level it is useful to recall that the magnetic properties of embedded clusters can be significantly modified by the interactions with the matrix if the hybrizations are strong. This is often the case for metallic substrates, particularly when the magnetic behaviour of the cluster and the matrix are very different. For example, very strong effects have been found in Co or Fe clusters embedded in $\mathrm{Nb}$ (non-magnetic) or in $\mathrm{Cr}$ (antiferromagnetic) [100,101]. In the latter case, one observes that if the size of the embedded Fe cluster is small, the magnetic order within the Fe cluster is antiferromagnetic with strongly reduced local magnetic moments $\mu(i)$. In other words, very small Fe clusters adopt the magnetic order induced by the spin density wave of the $\mathrm{Cr}$ matrix. This contrasts with the behaviour observed for free clusters which show ferromagnetic order and $\mu(i)>\mu_{\mathrm{b}}$. For $N \geq 6$, one observes the expected transition from antiferromagnetic to ferromagnetic order within $\mathrm{Fe}_{N}$. However, close to the interface with the matrix the $\mu(i)$ are significantly smaller than $\mu_{\mathrm{b}}$. The magnetic moments $\mu_{\mathrm{Fe}}(i)$ of the Fe atoms are very sensitive to the local chemical environment. In fact, $\left\langle S_{i z}\right\rangle_{\mathrm{Fe}}$ is found to be roughly proportional to the number of Fe atoms $z_{\mathrm{Fe}}(i)$ found in the first $\mathrm{NN}$ shell of atom $i$. Moreover, the average magnetization per atom is always smaller than $\mu_{\mathrm{b}}(\mathrm{Fe})$ due to the contributions of interface $\mathrm{Fe}$ atoms. The trend is thus opposite to that of free clusters or infinite surfaces. Similarly strong effects are 
observed in the case of Co clusters in $\mathrm{Nb}$, where the magnetic moments of the Co atoms at the $\mathrm{Co} / \mathrm{Nb}$ interface are simply quenched due to strong hybridizations with the environment. In contrast, in the case of inert matrices or colloidal solutions the properties of embedded clusters often resemble those of free clusters in many respects. Clusters on surfaces experience both the reduction of the local coordination number, as free clusters, and the cluster-substrate interactions, as embedded clusters. A variety of remarkable magnetic behaviours result from these competing effects. These are subjects of mayor current interest which shall be discussed in some detail in following sections.

\subsection{Relativistic contributions}

So far we have discussed the magnetic properties of clusters at a purely non-relativistic (NR) level. In NR quantum mechanics, the total spin operator $\vec{S}=\sum_{i} \vec{S}_{i}$ commutes with the Hamiltonian operator and therefore the direction of the magnetization relative to the cluster structure plays no role in the electronic properties. Thus, in the absence of an external magnetic field the NR ground-state and excited-state energies are independent of the direction of the magnetization. However, the electronic and magnetic properties of real ferromagnets do depend to some extent on the orientation of the magnetization with respect to the crystal structure and to the external shape of the system. The anisotropy of the ground-state energy (or of the free energy at $T>0$ ) is one of the main characteristics of a magnetic material. It determines the low-temperature orientation of the magnetization with respect to the geometry of the system and the stability of the magnetization direction in the case of single-domain particles. The magnetic anisotropy energy-defined as the energy difference involved in changing the magnetization direction from the low-energy direction or easy axis to a high-energy direction or hard axis - is a property of crucial importance in technological applications (e.g. magnetic recording or memory devices) where the magnetization must be pinned to a given direction in space.

The magneto-anisotropic behaviour is the result of relativistic corrections to the single-electron dynamics (Dirac equation) and to the electron-electron interaction (Breit interaction) (see, for instance, [102]). The leading terms are of second order in the ratio $v / c$ between the velocity of the electrons and the velocity of light. The relativistic corrections to the electron-electron interaction have two origins. First, the electrons are moving charges and therefore each electron interacts with the magnetic field generated by the current and by the spins of the other electrons. Second, the electromagnetic interactions are mediated by photons which travel at the speed of light and are thus affected by retardation effects. In the Hartree approximation to the Breit interaction, the magnetic dipole-dipole energy $E_{\mathrm{DD}}$ is given in terms of the magnetization density $\vec{m}(r)=\langle\hat{\vec{m}}(r)\rangle[102,103]$. For transition metals $E_{\mathrm{DD}}$ may be obtained as the sum of the dipole-dipole interactions between the local magnetic moments $\vec{\mu}_{i}$ at each atom $i$ :

$$
E_{\mathrm{DD}}=\frac{\mu_{\mathrm{B}}^{2}}{2} \sum_{i \neq j}\left[\frac{\vec{\mu}_{i} \cdot \vec{\mu}_{j}}{R_{i j}^{3}}-3 \frac{\left(\vec{R}_{i j} \cdot \vec{\mu}_{i}\right)\left(\overrightarrow{R_{i j}} \cdot \vec{\mu}_{j}\right)}{R_{i j}^{5}}\right] .
$$

The dipole-dipole interaction energy between pairs of local moments is very small even at NN distances $\left(E_{\mathrm{DD}} \simeq 3 \times 10^{-6} \mathrm{eV}\right.$ for $R_{0} \simeq 2.5 \AA$ and $\left.\mu \simeq 1 \mu_{\mathrm{B}}\right)$. However, $E_{\mathrm{DD}}$ decreases slowly as a function of distance $\left(E_{\mathrm{DD}} \sim 1 / R_{i j}^{3}\right)$ and the summation over pairs $i j$ converges very slowly. Therefore, $E_{\mathrm{DD}}$ depends on the shape of the nanoparticles and on the relative orientations of the magnetic moments in a nanostructure. 
The other relativistic corrections to the Schrödinger equation come from the single-electron dynamics which is ruled by the Dirac equation. The non-relativistic limit of the Dirac equation-including the terms up to the order $(v / c)^{2}$-is the Pauli Hamiltonian $H_{\text {Pauli }}=H_{\mathrm{NR}}+H_{\mathrm{SR}}+H_{\mathrm{SO}}$ [102]. One distinguishes, on the one side, the non-relativistic (Schrödinger) term $H_{\mathrm{NR}}$ and the scalar relativistic corrections $H_{\mathrm{SR}}$, usually included in the external (pseudo)potential that defines the hopping integrals (see Eq. (3)). On the other side, one finds the spin-orbit interactions:

$$
H_{\mathrm{SO}}=\frac{\hbar^{2}}{2 m^{2} c^{2} r} \frac{\partial V}{\partial r} \vec{\ell} \cdot \vec{s}=\alpha(r) \vec{\ell} \cdot \vec{s}
$$

which are qualitatively important since they modify the symmetry of the wavefunction [104]. The spinorbit coupling represents the interaction of the magnetic moment of the electron with the magnetic field that results from the electronic motion relative to the lattice potential $V(r)$. Since the electric field is strongest close to the nuclei, the SO effects are most important for the heaviest elements and for the most localized orbitals (core-electron states and $\mathrm{d}$ or f valence orbitals). For the d-electrons in transition metals it is a good approximation to consider only the intra-atomic terms. Thus, $H_{\mathrm{SO}}$ may be expressed as:

$$
H_{\mathrm{SO}}=-\sum_{i, \alpha \sigma, \beta \sigma^{\prime}} \xi_{i}\left(\vec{L}_{i} \cdot \vec{S}_{i}\right)_{\alpha \sigma, \beta \sigma^{\prime}} \hat{c}_{i \alpha \sigma}^{+} \hat{c}_{i \beta \sigma^{\prime}},
$$

where $\xi_{i}>0$ is the SO coupling constant at atom $i$, which is element-specific (e.g., $\xi_{i}=\xi_{\text {Co }}$ or $\xi_{\text {Pd }}$ ). $\left(\vec{L}_{i} \cdot \vec{S}_{i}\right)_{\alpha \sigma, \beta \sigma^{\prime}}$ stand for the matrix elements of $\vec{\ell} \cdot \vec{s}$ among the $\mathrm{d}$ states, that couple the up and down spinmanifolds depending on the relative orientation of the magnetization with respect to the lattice structure.

The redistributions of the spin-polarized electronic density which follow the specific local environment of different atoms are crucial for the properties of itinerant electrons in nanostructures. Since the spin-orbit (SO) interactions are very sensitive to the details of the electronic spectrum, the properties related to SO interactions (e.g. orbital moments and MAEs) depend significantly on the spin density distribution and on the variables that define it. For this reason, it is meaningful to perform accurate selfconsistent calculations for each orientation of the magnetization and in particular to derive the MAE in a non-perturbative fashion as difference between electronic energies. In the following, a self-consistent tight-binding approach is considered, as extended to include SO interactions [85]. The Hamiltonian is given by $H=H_{0}+H_{\mathrm{C}}+H_{\mathrm{SO}}$, where the inter-atomic hopping term $H_{0}$ and the Coulomb interaction term $H_{\mathrm{C}}$ are the same as in Eqs. (3) and (5), and the SO coupling term $H_{\mathrm{SO}}$ is given by Eq. (11). The average d-electron occupation at site $i$ :

$$
n(i)=\sum_{\alpha}\left(\left\langle\hat{n}_{i \alpha \uparrow}\right\rangle+\left\langle\hat{n}_{i \alpha \downarrow}\right\rangle\right)
$$

and the local spin magnetic moment:

$$
\left\langle S_{i \delta}\right\rangle=\frac{1}{2} \sum_{\alpha}\left(\left\langle\hat{n}_{i \alpha \uparrow}\right\rangle-\left\langle\hat{n}_{i \alpha \downarrow}\right\rangle\right),
$$

are calculated self-consistently by integrating the local densities of states (LDOS) $\rho_{i \alpha \sigma}^{\delta}(\varepsilon)$ up to the Fermi energy $\varepsilon_{\mathrm{F}}$. Since $\rho_{i \alpha \sigma}^{\delta}$ depends on the magnetization direction $\delta$, it is determined by performing independent calculations for each orientation of $\vec{M}$. In this way, the effects of hybridization, Coulomb, and SO interactions are treated on the same footing. 
Once self-consistency is achieved, the average local orbital moments $\left\langle L_{i \delta}\right\rangle$ at atom $i$ along the magnetization direction $\delta$ are calculated from:

$$
\left\langle L_{i \delta}\right\rangle=\sum_{\sigma} \sum_{m=-2}^{2} \int_{-\infty}^{\varepsilon_{\mathrm{F}}} m \rho_{i m \sigma}^{\delta}(\varepsilon) \mathrm{d} \varepsilon
$$

where $m$ indicates the magnetic quantum number. For simplicity, the quantization axis of the orbital momentum is here taken to be the same as the spin-quantization axis. The other components of $\left\langle\vec{L}_{i}\right\rangle$ can be obtained in an analogous way by rotating the orbital quantization direction. The cluster average of $\left\langle L_{\delta}\right\rangle=\left(\sum_{i=1}^{N}\left\langle L_{i \delta}\right\rangle\right) / N$ can be compared with the outcome of XMCD experiments (cf. Section 5).

The electronic energy $E_{\delta}=\sum_{i} E_{\delta}(i)$ can be written as the sum of local contributions:

$$
E_{\delta}(i)=\sum_{\alpha \sigma}\left[\int_{-\infty}^{\varepsilon_{\mathrm{F}}} \varepsilon \rho_{i \alpha \sigma}^{\delta}(\varepsilon) \mathrm{d} \varepsilon-E_{i \alpha \sigma}^{\mathrm{dc}}\right],
$$

corresponding to the different atoms $i$ of the deposited cluster and its environment. Here, $E_{i \alpha \sigma}^{\mathrm{dc}}=$ $(1 / 2) \Delta \varepsilon_{i \sigma}\left\langle\hat{n}_{i \alpha \sigma}\right\rangle$ stands for the double-counting correction. The MAE is defined as the change $\Delta E$ in the electronic energy $E_{\delta}$ associated to a change in the orientation of the magnetization. In the case of deposited clusters, one usually considers the direction $\delta=z$ perpendicular to the surface and two directions within the surface plane: $\delta=x$ along a nearest-neighbour bond and $\delta=y$ perpendicular to $\hat{x}$. Thus, positive (negative) values of the off-plane anisotropy energy $\Delta E_{x z}=E_{x}-E_{z}$ indicate a perpendicular easy (hard) axis. Taking advantage of the local formulation one may express $\Delta E_{x z}=\sum_{i} \Delta E_{x z}(i)$ as a sum of atom-resolved contributions:

$$
\Delta E_{x z}(i)=E_{x}(i)-E_{z}(i)
$$

where $E_{\delta}(i)$ is given by Eq. (15). Thus, the magneto-anisotropic properties can be related to the various local atomic environments. Analogous expressions hold for the in-plane anisotropy energy $\Delta E_{x y}=E_{x}-E_{y}$. Notice that the calculation of $\Delta E$ as difference of $E_{\delta}$ 's is a non-perturbative approach that includes in particular the effects of spin density redistributions resulting from SO interactions. This requires a very precise determination of $E_{\delta}$ and of the self-consistent equations, since $\Delta E$ is usually a small quantity of the order of a meV.

A simple physical picture for the MAE and its relation to the orbital moments can be derived for homogeneous systems in the limit of saturated magnetic moments with a large exchange splitting $\varepsilon_{i d} \uparrow-$ $\varepsilon_{i d \downarrow}=J \mu$ [105]. In this case, at lowest-order perturbation theory, we may neglect the terms that mix spin-up and spin-down states. Thus, $H_{\mathrm{SO}} \simeq(\xi / 2) \sum_{i}\left(\hat{\ell}_{i z \uparrow}-\hat{\ell}_{i z \downarrow}\right)$ can be regarded as a magnetic field acting on the orbital moment $\hat{\ell}_{i z \sigma}$. Moreover, $\left\langle H_{\mathrm{SO}}\right\rangle / N_{\mathrm{a}}=(\xi / 2)\left(\left\langle L_{z \uparrow}\right\rangle-\left\langle L_{z \downarrow}\right\rangle\right)$, where $\left\langle L_{z \sigma}\right\rangle$ is the average orbital moment per atom. Taking the up spins as the majority ones, we have $\left\langle L_{z \downarrow}\right\rangle \simeq 0$ for $n_{\mathrm{d}}<5$, and $\left\langle L_{z \uparrow}\right\rangle \simeq 0$ for $n_{\mathrm{d}}>5$. Thus, $\left\langle H_{\mathrm{SO}}\right\rangle / N_{\mathrm{a}} \simeq \pm(\xi / 2)\left\langle L_{z}\right\rangle$, where the $+(-)$ sign corresponds to $n_{\mathrm{d}}<5$ $\left(n_{\mathrm{d}}>5\right)$. As in the atom, antiparallel (parallel) alignment of $\left\langle L_{z}\right\rangle$ and $\left\langle S_{z}\right\rangle$ is favoured for $n_{\mathrm{d}}<5\left(n_{\mathrm{d}}>5\right)$. Under the previous assumptions, the lowest-energy magnetization direction (easy axis) is the one yielding the largest orbital moment. In other words, the stable configuration is obtained when the spin magnetization is "turned" to the direction yielding the largest $|\langle\vec{L}\rangle|$. 


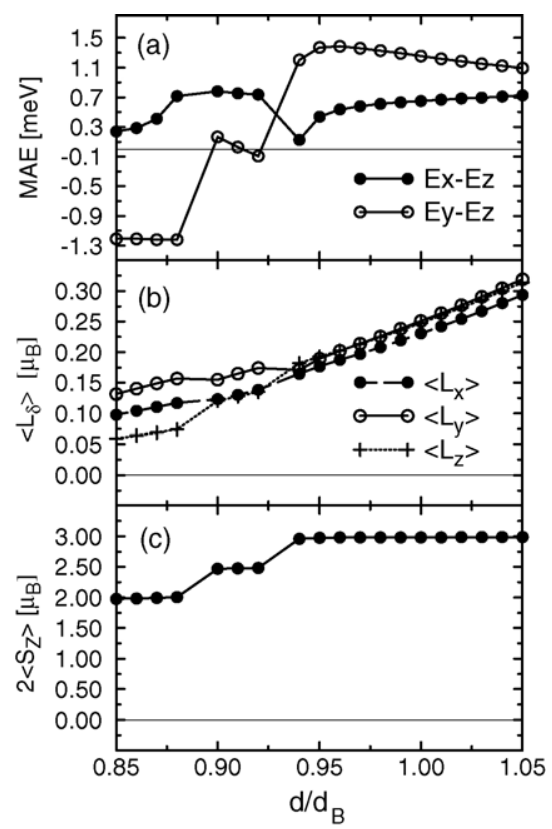

Fig. 14. (a) Magnetic anisotropy energy (MAE), (b) orbital magnetic moment $\left\langle L_{\delta}\right\rangle$ and (c) spin magnetic moment $\left\langle S_{z}\right\rangle$ of $\mathrm{Fe}_{4}$ with rhombohedral structure as a function of the bond length $d\left(d_{\mathrm{B}}=\right.$ bulk NN distance). The magnetization direction $\delta=x$ is along the middle bond, the direction $\delta=y$ is perpendicular to $x$ and within the plane of the cluster, and $\delta=z$ is perpendicular to $x$ and $y[85,106]$.

\subsection{Magnetic anisotropy of small clusters}

In Fig. 14, results are given for the MAE $\Delta E$, the orbital angular momentum $\left\langle L_{\delta}\right\rangle$ along the magnetization direction $\delta(\delta=x, y, z)$, and the average spin projection $\left\langle S_{z}\right\rangle$ of an $\mathrm{Fe}_{4}$ cluster with rhombohedral structure [106]. The results are given as a function of the bond length $d$ in order to analyze the role of cluster relaxation and to infer the possible coupling of the magnetization direction to vibrations and distortions. Only $\left\langle S_{z}\right\rangle$ is shown, since the magnitude of the spin magnetization $|\langle\vec{S}\rangle|$ depends very weakly on the considered direction (typically, $\left|\left\langle S_{z}\right\rangle-\left\langle S_{x}\right\rangle\right| \sim 10^{-3}$ to $10^{-4}$ ). Comparing Figs. $14 \mathrm{a}$ and $\mathrm{c}$, it is clear that the variations of the MAE are related to the variations of $\left\langle S_{z}\right\rangle$ and to the resulting changes in the electronic spectrum. For large values of $d / d_{\mathrm{B}}$, the spin magnetic moments are saturated, i.e. $\left\langle S_{z}\right\rangle \simeq\left(10-v_{\mathrm{d}}\right) / 2=3 / 2$. When $d / d_{\mathrm{B}}$ decreases, discrete changes in the spin polarization occur and non-saturated moments are obtained (see Section 3.2). For constant values of $\left\langle S_{z}\right\rangle$, the MAE and orbital momentum $\left\langle L_{\delta}\right\rangle$ vary continuously, since the electronic spectrum and the local magnetic moments are continuous functions of $d / d_{\mathrm{B}}$. However, the situation is different when $\left\langle S_{z}\right\rangle$ changes, since a strong and discontinuous redistribution of the spin-polarized density takes place. In this case, important changes in the energy-level structure occur around $\varepsilon_{\mathrm{F}}$ that modify the details of the SO mixing. The resulting changes in the electronic energy depend on the explicit form of $H_{\text {SO }}$ and therefore on the direction of the magnetization (see Fig. 14). Consequently, very significant and discontinuous variations of the MAE are observed which may even lead to a change of sign of the MAE. Notice, that the rhombohedral $\mathrm{Fe}_{4}$ cluster presents a remarkable in-plane anisotropy $\left(E_{x}-E_{y}\right) \simeq 0.4-1.4 \mathrm{meV}$. This 
Table 1

Size and structural dependence of the magnetic anisotropy energy (MAE) of $\mathrm{Fe}_{N}$ clusters [85]

\begin{tabular}{llccr}
\hline$N$ & Structure & $\Delta E\left(d / d_{\mathrm{B}}=1.05\right)$ & $\Delta E\left(d / d_{\mathrm{B}}=1.00\right)$ & $\Delta E\left(d / d_{\mathrm{B}}=0.90\right)$ \\
\hline 3 & (a) & $5.13(1.43)$ & $5.30(-1.01)$ & $-0.11(-0.10)$ \\
& (b) & 2.01 & 1.69 & 1.25 \\
4 & (c) & $5.02(-0.48)$ & $5.21(-0.59)$ & $-1.03(-0.11)$ \\
& (d) & $0.30(-0.12)$ & $0.27(-0.12)$ & $0.33(0.31)$ \\
5 & (e) & $0.29(-1.01)$ & $0.28(-0.82)$ & $-0.10(-0.75)$ \\
& (f) & $1.09(-0.01)$ & $0.88(-0.03)$ & $-0.07(0.04)$ \\
6 & (g) & $-1.25(-0.76)$ & $-1.12(-0.62)$ & $0.33(-0.29)$ \\
& (h) & $4.66(0.04)$ & $4.82(-0.02)$ & $-0.07(-0.23)$ \\
7 & (i) & $1.78(0.40)$ & $1.91(0.09)$ & $2.22(0.30)$ \\
& (j) & $4.32(-0.03)$ & $4.52(-0.02)$ & $-0.39(0.00)$ \\
\hline
\end{tabular}

The off-plane MAE $\Delta E=E_{x}-E_{z}$ and the in-plane MAE $\Delta E=E_{x}-E_{y}$ (results in brackets) are given in meV for different values of the inter-atomic bond length $d\left(d_{\mathrm{B}}=\right.$ bulk NN distance). Different structures are considered for each cluster size: (a) triangle, (b) chain, (c) square, (d) rhombus, (e) trust, (f) square pyramid, (g) triangle, (h) square bipyramid, (i) hexagon, and (j) pentagonal bipyramid (see [106]).

indicates that uniaxial anisotropy models are not directly applicable to clusters that have strongly reduced symmetry. A similar behaviour is found for other clusters and band-fillings [106].

Table 1 shows results for the MAE of small Fe clusters with different structures and inter-atomic distances [106]. The main general trends may be summarized as follows:

(i) The MAE is much larger in small clusters than in the corresponding crystalline solids. In fact, the anisotropy energy $\Delta E$ is often even larger than in thin films. For instance, $\Delta E \sim 4-5 \mathrm{meV}$ is a typical value for clusters (see Table 1). This is in agreement with experiments on free clusters and supported Fe nanoparticles [107].

(ii) $\Delta E$ depends much more sensitively than the spin moments on the geometrical structure of the cluster. Indeed, changes of sign in $\Delta E$ are found as a function of the inter-atomic distance $d$ even in situations where the magnetic moments are nearly saturated and therefore do not depend on the cluster structure.

(iii) The magnetic energy surface of low symmetry clusters is in general quite complex. In particular, the in-plane MAEs are often found to be very important. In some cases they are even larger than the usually considered off-plane anisotropy. The in-plane MAE is of course largest for low-symmetry structures and decreases, though not monotonically, as the angle between non-equivalent $x$ - and $y$ directions decreases. Experiments support these conclusions [107-110].

\subsection{Size-dependent enhancement of orbital magnetism}

In atoms, Hund's rules predict maximum orbital angular moment $L$ compatible with maximum spin multiplicity, while in transition metal solids, electron delocalization and band formation result in an almost complete quenching of $\langle L\rangle$. Such intrinsic differences between atomic and bulk behaviours are characteristic of systems developing itinerant-electron magnetism. Consequently, investigations of orbital magnetism in TM clusters-in the way from the atom to the solid-should reveal novel size- 


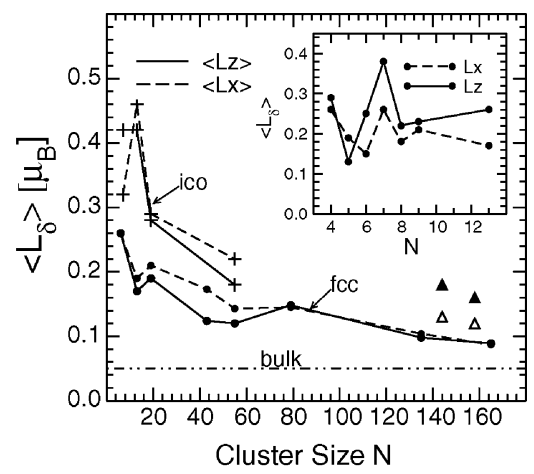

Fig. 15. Average orbital magnetic moment per atom $\left\langle L_{\delta}\right\rangle$ of $\mathrm{Ni}_{N}$ clusters with fcc-like (dots) and icosahedral-like structures (crosses) $[34,85]$. The magnetization direction $\delta$ is a principal $C_{n}$ symmetry axis $(\delta=z)$ or a NN bond perpendicular to $z(\delta=x)$. Filled (open) triangles refer to coin-like bilayer clusters with perpendicular (in-plane) magnetization. Results for small clusters are given in the inset.

dependent phenomena that are important both from a fundamental standpoint and in view of applications of cluster-based magnetic nanostructures. These general considerations already indicate that $\langle L\rangle$ should be very sensitive to the local environment of the atoms. Indeed, recent experiments on chains [111] and clusters at surfaces [33,112-114] show that $\langle L\rangle$ is typically a factor 2-5 larger than in the corresponding solids. Calculations on small supported clusters confirm these conclusions [115,116]. Moreover, a sizedependent enhancement of $\langle L\rangle$ should have direct consequences on the results for the average magnetic moments per atom and on the comparison between theory and experiment [10,20,117]. It is therefore important to understand the dependence of orbital magnetism on variables like size, structure, local atomic environment, and d-band filling.

In order to analyze the crossover from atomic $L$ to bulk-like quenching of $\langle L\rangle$ with increasing size we focus first of all on $\mathrm{Ni}_{N}$ clusters. Besides its experimental relevance, $\mathrm{Ni}$ is a very interesting system since the differences between atomic and bulk orbital moments are dramatic $\left(L=L_{\mathrm{a}}=2 \mu_{\mathrm{B}}\right.$ in the atomic s ${ }^{1} \mathrm{~d}^{9}$ configuration, and $\langle L\rangle=L_{\mathrm{b}}=0.05 \mu_{\mathrm{B}}$ in the solid). In addition, the spin moments in $\mathrm{Ni}_{N}$ are the smallest among the ferromagnetic $3 \mathrm{~d}$ TMs, and therefore the contribution of $\langle L\rangle$ to the total magnetic moment $\langle\vec{M}\rangle=2\langle\vec{S}\rangle+\langle\vec{L}\rangle$ is expected to be particularly significant. In Fig. 15, results are given for the average orbital moment per atom $\left\langle L_{\delta}\right\rangle=\left[\sum_{i=1}^{N} L_{\delta}(i)\right] / N$ of $\mathrm{Ni}_{N}$ with $N \leq 165$, having face-centered cubic or icosahedral-like geometries. Low-symmetry structures and two-dimensional (2D) islands are also considered. These are representative of different local symmetries and shapes and allow to quantify the role of cluster structure on orbital magnetism. On the one hand, we observe that the reduction of system size causes a remarkable enhancement of $\left\langle L_{\delta}\right\rangle$ with respect to the solid. Values about six to eight times larger than $L_{\mathrm{b}}=0.05 \mu_{\mathrm{B}}$ are not uncommon. On the other hand, comparison with the atomic result $L_{\mathrm{a}}=2 \mu_{\mathrm{B}}$ shows that the largest part of the quenching of $L$ takes place already at the smallest clusters, as soon as full rotational symmetry is lost. For example, for $\mathrm{Ni}_{3}$ (triangle) we obtain $\left\langle L_{z}\right\rangle=0.47 \mu_{\mathrm{B}}$, and for $\mathrm{Ni}_{4}$ (rhombus) $\left\langle L_{z}\right\rangle=0.35 \mu_{\mathrm{B}}$. Concerning the size dependence, one observes that $\left\langle L_{\delta}\right\rangle$ decreases with increasing $N$ showing some oscillations as bulk-like quenching is approached (see Fig. 15). Notice that an important enhancement of $\left\langle L_{\delta}\right\rangle$, about $100 \%$, is still present even for the largest considered sizes (e.g. $\left\langle L_{\delta}\right\rangle / L_{\mathrm{b}}=1.8$ for $\left.N=165\right)$. In smaller clusters, $\left\langle L_{\delta}\right\rangle / L_{\mathrm{b}}$ ranges from $\left\langle L_{\delta}\right\rangle / L_{\mathrm{b}} \simeq 3$ for $N=50-80$, to $\left\langle L_{\delta}\right\rangle / L_{\mathrm{b}} \simeq 5$ for $N=10-20$. Already at this stage one concludes that orbital magnetism is the source of an important contribution to the size-dependent magnetic properties of Ni clusters. 
In Fig. 15, results are also given for icosahedral clusters having $N \leq 55$ atoms. $\left\langle L_{\delta}\right\rangle$ decreases with increasing $N$ in a similar way as for the fcc geometries. Nevertheless, the five-fold symmetric structures yield in general significantly larger $\left\langle L_{\delta}\right\rangle$ than the cubic symmetric ones. The results for $N \geq 13$ concern mainly highly-symmetric structures with nearly spherical shape and closed nearest-neighbour shells. Clusters with lower symmetry usually present a very rich size dependence, particularly when the proportion of surface atoms is large. This is illustrated by the results for $N \leq 13$ given in the inset, which correspond to optimized geometries derived form ab initio calculations [118-120].

Structural changes may also affect the convergence to bulk-like quenching for large $N$, particularly for clusters on surfaces, which shape can be experimentally tuned at least to some extent $[33,111,113,114]$. This is illustrated by the results for two-layer-thick coin-like $\mathrm{Ni}_{N}$, that are indicated by the triangles in Fig. $15\left(N=144\right.$ and 158). For these 2D islands, $\left\langle L_{\delta}\right\rangle$ is much larger than for 3D fcc clusters of comparable size. The enhancement with respect to $L_{\mathrm{b}}$ is here about a factor of 3 . Similar trends are found for other TMs, in qualitative agreement with recent experiments on Fe and Co particles (see Section 5.1 and $[33,111,113,114])$. Structure and shape can have a strong influence on the quantitative value of the orbital moments in TM nanoparticles, even though the main trends are not affected significantly by the precise geometry.

From the solid state perspective, the enhancement of $\left\langle L_{\delta}\right\rangle$ can be qualitatively understood as the result of the following major contributions that are related to the changes in the local environment of the atoms:

(i) The reduction of local coordination number with decreasing $N$ causes an increase of the local spin polarizations $\left\langle S_{i \delta}\right\rangle$ which induces larger orbital moments by means of the spin-orbit interactions.

(ii) The orbital dependence of intra-atomic Coulomb interaction favours the occupation of high- $m$ states thereby amplifying the enhancement of $\left\langle L_{\delta}\right\rangle$. Although this contribution is quantitatively important, typically $30-40 \%$ of the value of $\left\langle L_{\delta}\right\rangle$, it does affect significantly the trends as a function of size and structure.

(iii) The presence of degeneracies in the single-particle spectrum allows a more effective spin-orbit mixing that enhances $\left\langle L_{i \delta}\right\rangle$ even in situations where $\left\langle S_{i \delta}\right\rangle$ is saturated.

Qualitatively, the size-dependent magnetic moments are determined by the competition between the kinetic energy, which favours electron delocalization and small moments, and the Coulomb interactions, which tend to suppress charge fluctuations and lead to Hund rules. Thus, low-spin and quenched- $L$ states become comparatively less stable than high-spin and enhanced- $L$ states as $N$ is reduced. Notice, moreover, that the changes in $\left\langle S_{i \delta}\right\rangle$ and $\left\langle L_{i \delta}\right\rangle$ involve different energy scales. Therefore, the occupations of the different $m$ orbitals may vary without altering the spin polarizations (e.g. compare icosahedral and fcc clusters in Fig. 15). Finally, one also observes less predictable effects related to the details of the electronic structure and its dependence on cluster geometry, like the presence of high-symmetry axes or changes in bond length.

The environment dependence of the local orbital moments $L_{\delta}(i)$ provides further insight on the enhancement of $\left\langle L_{\delta}\right\rangle$ in small clusters, and on the development of bulk-like quenching for large $N$. One observes that $\left\langle L_{i \delta}\right\rangle$ generally increases with $i$, showing some oscillations as we move from the center to the surface of the cluster. Therefore, the enhancement of $\left\langle L_{\delta}\right\rangle$ is driven by the surface, as it is the case for the spin moments [85]. A similar behaviour is found for icosahedral geometries. For small $N$ (e.g. $N=19$ and 55), the enhancement of $\left\langle L_{i \delta}\right\rangle$ concerns practically all atoms, including those with a complete NN shell. For larger sizes $(N \geq 135)$, bulk-like quenching starts to set in at the interior of the cluster, leaving significantly 


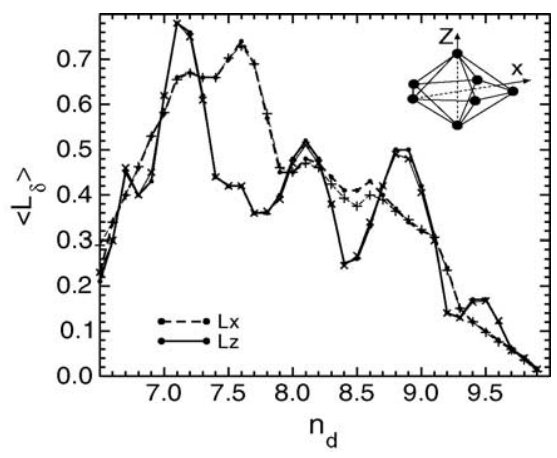

Fig. 16. Average orbital moment per atom $\left\langle L_{\delta}\right\rangle$ in a seven-atom TM cluster as a function of d-band filling $n_{\mathrm{d}}$. The structure and magnetization directions $\delta$ are illustrated.

enhanced orbital moments only at a few outermost shells. As expected, one approaches the behaviour found for TM surfaces, where the enhanced $\left\langle L_{i}\right\rangle$ are restricted to a few uppermost layers. A droplet model, i.e. $\left(\left\langle L_{\delta}\right\rangle-L_{\mathrm{b}}\right) \propto N^{-1 / 3}$, is in fact a good first approximation for $N \geq 150-200$, although the convergence to $L_{\mathrm{b}}$ remains rather slow. The fact that $\left\langle L_{i \delta}\right\rangle \simeq L_{\mathrm{b}}$ for inner atoms indicates that cubic point-group symmetry, absent in all atoms except the central one, is not essential for reaching nearly bulk-like quenching. Instead, the recovery of a bulk-like local atomic environment seems a more appropriate interpretation.

The contribution of the orbital moments - that in Ni align parallel to the spin moments-increases the value of the total magnetic moment $\bar{\mu}_{N}=\langle L\rangle+2\langle S\rangle$ predicted by theory, thereby improving the agreement with experiment. In fact, despite some quantitative differences among the results obtained by different groups, available spin-only calculations yield ground-state spin magnetizations that underestimate systematically the experimental $\bar{\mu}_{N}$ by about $0.3-0.6 \mu_{\mathrm{B}}$ for $N \leq 13$ [118-120]. The largest part of this discrepancy is removed by including the enhanced orbital contributions, thereby resolving the previous controversy on this subject. The remaining differences, about $(0.1-0.2) \mu_{\mathrm{B}}$, are not far from the estimated experimental uncertainties. This could also be related to correlation effects, which should favour electron localization leading to more atomic-like orbital moments and possibly to a further enhancement of $\left\langle L_{\delta}\right\rangle$.

The trends for other TMs can be inferred from Fig. 16, where $\left\langle L_{\delta}\right\rangle$ of a pentagonal bipyramid is given as a function of d-band filling $n_{\mathrm{d}}$. $\left\langle L_{\delta}\right\rangle$ increases approximately linearly with increasing number of $\mathrm{d}$ holes, as we move from $\mathrm{Ni}\left(n_{\mathrm{d}} \simeq 9\right)$ to $\mathrm{Fe}\left(n_{\mathrm{d}} \simeq 7\right)$. Notice that the orbital moment per $\mathrm{d}$ hole $\left\langle L_{\delta}\right\rangle /(10-$ $\left.n_{\mathrm{d}}\right) \simeq(0.25-0.3) \mu_{\mathrm{B}}$ is quite close to recent experimental results on $\mathrm{Fe}_{N}$ clusters $(N \leq 9)$ deposited on $\mathrm{Ni} / \mathrm{Cu}\left(\begin{array}{lll}0 & 0 & 1\end{array}\right)$ [114]. In addition one observes oscillations and changes of sign of the anisotropy $\Delta L=L_{z}-L_{x}$, which are associated to changes in the occupations of molecular states $k$ with different orbital moments $\langle l\rangle_{k}$. This is consistent with previously observed oscillations of the magnetic anisotropy energy as function of $n_{\mathrm{d}}$ [106]. One concludes that the size-dependent enhancement of $\left\langle L_{\delta}\right\rangle$ is important for all magnetic TM clusters.

\section{Magnetic characterization techniques}

The experimental methods employed to carry out the magnetic measurements will be described in this section. Although magnetic characterization methods have been developed since the invention of the 
compass in the 12th century AD, and now constitute a vast number, the techniques relevant to this review must be able to measure the magnetization in tiny amounts of cluster-assembled material. In addition, the measurement usually has to be carried out in situ in UHV. The description focuses thus on those techniques that work under one or both of these restrictions.

\subsection{Vibrating sample magnetometry}

Vibrating sample magnetometry VSM is a standard technique for measuring the magnetic moment in materials that was originally described by Foner [121] over 40 years ago. The magnetic moment of a sample is determined by vibrating it between conducting coils and measuring the alternating voltage developed at the same frequency. The sensitivity of the technique has steadily improved and modern instruments can measure moments as low as $10^{-7} \mathrm{~A} \mathrm{~m}^{2}$, corresponding to $\approx 10^{16} \mathrm{Fe}$ atoms. Recently, a system that allows in situ measurements on exposed clusters in UHV has been demonstrated [122]. Clusters are deposited onto a substrate and then sealed, in their UHV environment, within an ampoule that can be withdrawn and inserted into the magnetometer without exposing the sample to atmosphere. The technique is not sensitive enough to measure significantly less than a single cluster layer so it cannot be used to study isolated particles. The ability to measure exposed layers is, however, important since even thick cluster films show a marked change in magnetic behaviour when coated with a protective nonmagnetic capping layer to allow removal from the vacuum for ex situ measurements [122].

\subsection{Magneto-optical Kerr effect in the visible regime}

In 1877, Kerr [123] observed that a ferromagnetic medium may effect the polarization or intensity of polarized light when reflected by its surface. This phenomenon called magneto-optical Kerr effect (MOKE) using visible light has become a standard tool for investigating magnetic systems. Moog and Bader applied this technique as a surface sensitive method in investigations of ultrathin magnetic films [124]. In the case of systems with dimensions below the wavelength of the light source, the already small magnitude of visible MOKE decreases again with the film thickness. Today, advanced experimental techniques allow a sensitivity of a few atomic monolayers and even clusters embedded in non-magnetic matrices [125-127]. In general, one distinguishes three different MOKE geometries shown in Fig. 17. Using linearly s- or p-polarized light in-plane or out-of-plane magnetization components can be analyzed depending on the experimental configuration. In the longitudinal case (left part of Fig. 17), an external magnetic field is applied in the in-plane configuration parallel to the plane of incidence. The respective in-plane magnetization component causes a rotation of the polarization plane combined with a change in the ellipticity which can easily be detected when using visible light. By varying the strength of the
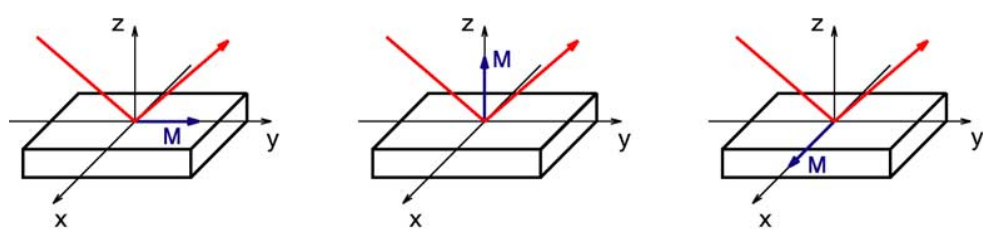

Fig. 17. Different experimental configurations of the magneto-optical Kerr effect: longitudinal (left part), polar (middle), and transverse MOKE set-up (right part). 
magnetic field it is therefore possible to probe the magnetization reversal during a hysteresis cycle with an in-plane oriented external magnetic field. The polar MOKE is quite similar but with the magnetic field applied perpendicularly to the surface (middle of Fig. 17). With light impinging nearly perpendicular to the surface one can measure mainly polar hysteresis loops, i.e. analyze the polar component of the magnetization. In contrast, an intensity change is observed in the transverse geometry (right part of Fig. 17). When using p-polarized light the intensity difference is proportional to the in-plane magnetization component perpendicular to the plane of incidence. This set-up opens the possibility to measure hysteresis loops without polarization analysis. It is therefore well suited for experiments in the soft X-ray regime where the analysis of the polarization in the reflected beam is much more complicated due to the fact that most materials absorb soft X-rays within a very short distance.

\subsection{Magnetic circular and linear dichroism in X-ray based spectroscopy techniques}

Using tuneable soft X-ray based spectroscopy techniques opens the way to perform element-specific investigations in a sample. Possible channels for detection are either photoelectrons emitted from the sample, the photoabsorption signal, and the detection of the specularly scattered X-rays. All of these methods are sensitive to magnetic phenomena provided that the incoming photon energy, its polarization, the magnetization direction, and the direction of the detected photoelectrons fulfil certain conditions on their relative orientations. Magnetic dichroic phenomena can be observed in the $3 p$ angle-resolved

(a) XMCD

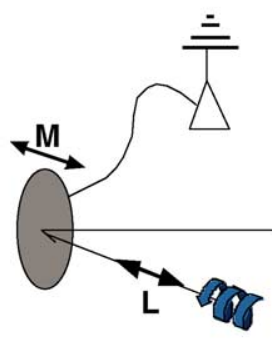

(b)

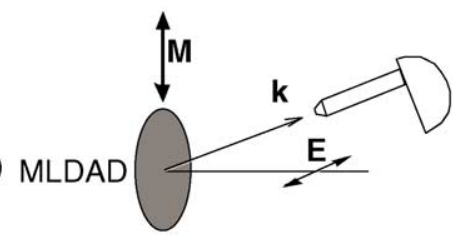

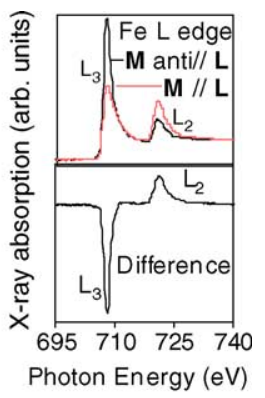

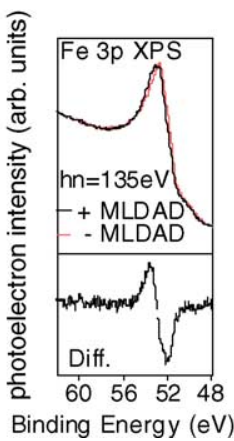

Fig. 18. (a) XMCD measures the difference in L-edge absorption (here, of Fe clusters on HOPG) between parallel and antiparallel alignment of the magnetization vector and photon spin. The magnetic field is applied along the direction of the photon beam and at a variable angle, $\theta$, relative to the sample normal. (b) MLDAD measures the difference in the Fe $3 p$ photoemission spectra taken with linearly polarized XUV light on reversing the magnetization in the sample plane and perpendicular to the photon polarization vector. 
photoemission spectra of the transition metals taken with linearly polarized light with the geometry shown in Fig. 18b. The technique measures the difference in the spectra in response to reversing the alignment between the in-plane sample magnetization and the linear polarization of the XUV light $[128,129]$. Switching the geometry in this way modifies the angular distribution of the photoelectrons but not the total current so the dichroism vanishes if the photoelectron current is collected at all emission angles. To observe it, the spectrum must be collected at a specific emission angle hence the technique is called magnetic linear dichroism in the angular distribution (MLDAD) [129,130]. Since angle-resolved photoemission spectra must be collected in zero field, the technique is, in general, restricted to measuring remanence. An advantage is the high surface sensitivity and its capability of measuring sub-monolayer quantities of a material. Schemes using magnetic substrates that expose the clusters to fields $B \approx 1 \mathrm{~T}$ but still allow photoemission measurements have also been developed [131]. As with XMCD, a synchrotron source must be used to provide the polarized XUV radiation. The only sources that emit a sufficiently high flux of circularly polarized X-rays to study nanostructures by XMCD are electron storage rings. These produce circularly polarized radiation of either helicity above and below the orbit plane of the electrons and also from insertion devices such as helical undulators [132].

\subsubsection{X-ray magnetic circular dichroism in photoabsorption}

The most common and powerful technique for studying magnetic behaviour at surfaces and in nanostructures is X-ray magnetic circular dichroism in photoabsorption (XMCD), a method that has been experimentally developed by Schütz et al. [133]. In the case of transition metals, it involves the measurement of the difference in L-edge X-ray absorption spectra obtained using circularly polarized Xrays with their angular momentum parallel or anti-parallel to the sample magnetization. The orbital $\left(m_{\mathrm{L}}\right)$ and spin $\left(m_{\mathrm{S}}\right)$ contributions to the total magnetic moment can be determined independently and (in heterogeneous systems) for each element by applying the sum rules to the absorption spectra $\sigma$ recorded for opposite photon spins $[134,135]$. The experimental geometry and an L-edge absorption spectrum taken from Fe clusters deposited on graphite [36] are shown in Fig. 18a. Specifically, the orbital and spin moments are related to the absorption spectra by:

$$
\begin{aligned}
& m_{\mathrm{L}}=\mu_{\mathrm{B}} \cdot\left\langle L_{z}\right\rangle=2 n_{\mathrm{h}} \frac{\int_{L_{3}+L_{2}} \sigma^{+}-\sigma^{-} \mathrm{d} E}{\int_{L_{3}+L_{2}} \sigma^{+}+\sigma^{0}+\sigma^{-} \mathrm{d} E}, \\
& m_{\mathrm{S}}=2 \mu_{\mathrm{B}}\left(\left\langle S_{z}\right\rangle+\frac{7}{2}\left\langle T_{z}\right\rangle\right)=\frac{3}{2} n_{\mathrm{h}} \frac{\int_{L_{3}} \sigma^{+}-\sigma^{-} \mathrm{d} E-2 \int_{L_{2}} \sigma^{+}-\sigma^{-} \mathrm{d} E}{\int_{L_{3}+L_{2}} \sigma^{+}+\sigma^{0}+\sigma^{-} \mathrm{d} E} .
\end{aligned}
$$

More precisely, the technique measures the quantities: $\left\langle L_{z}\right\rangle$ and $\left\langle S_{z}+7 T_{z}\right\rangle$ per valence band hole, $n_{\mathrm{h}}$, where $\left\langle L_{z}\right\rangle$ and $\left\langle S_{z}\right\rangle$ are the expectation values of the $z$ (static) components of the orbital and spin angular momenta of the atoms and $\left\langle T_{z}\right\rangle$ is the expectation value of the magnetic dipole operator $\langle T\rangle$. This averages to zero over all directions and does not appear in conventional magnetic measurements. Since the X-rays sample a directional cut through the atomic electron density, the term must be included in the sum rule. It can be eliminated, yielding the pure spin moment, by averaging measurements over all X-ray incidence angles, making a single measurement on a polycrystalline sample or, for samples with rotational symmetry about the substrate normal, making a single measurement at the 'magic' angle $[136,137]$. The absorption spectrum $\sigma^{0}$ requires in principle linearly polarized radiation, but is usually substituted by $\sigma^{0}=1 / 2\left(\sigma^{+}+\sigma^{-}\right)$. A more convenient procedure is described by Chen et al. [138]. It enables easy access to the ratio of the orbital and spin moment $m_{\mathrm{L}} / m_{\mathrm{S}}$ by calculating only the intensity 
difference (i.e. the dichroic) spectrum $\sigma_{\mathrm{XMCD}}=\sigma^{+}-\sigma^{-}$. Of course, the individual spin and orbital moments can also be determined. One should generally take into account, that the sum rules are based on the atomic model picture and neglect band structure effects in the final states as well as the polarization from s- and p-electrons. For the $3 \mathrm{~d}$ metals, only contributions from the $3 \mathrm{~d}$-electrons are included into the spin sum rule. In a rough estimate the error by neglecting the $4 \mathrm{~s}-$ and $4 \mathrm{p}$-electrons is in the order of less than $5 \%$ or $0.1 \mu_{\mathrm{B}}$, respectively.

A short remark should be made regarding the accuracy of XMCD measurements on nanoparticles. Usually, photoabsorption spectra are obtained by measuring the total electron yield (TEY), i.e. the drain current from the sample generated by the photoabsorption process. The TEY signal deviates from the real absorption strength and thus affects the evaluation of the orbital and spin moments when applying the sum rules $[139,140]$. In general, the so-called self-absorption leads to an underestimation of the spin and, even more, the orbital moment depending on the angle of incidence. This phenomenon becomes important for films thicker than $2 \mathrm{~nm}$ and bulk materials. For these materials, corrections can be carried out when using the expression given in [139]. The procedure is more difficult to perform in the case of nanoparticles and has recently been addressed by means of a Monte Carlo simulation [141]. This work shows that self-absorption effects have to be included for clusters larger than $3 \mathrm{~nm}$. For example, in Fe clusters with a size of $10 \mathrm{~nm}$, the orbital moments may be underestimated by a factor of 0.63 , while the spin moments are only reduced by a factor of 0.95 .

The finite escape depth of the detected electrons (typically 1.5-1.7 nm for Fe and 2.2-2.5 $\mathrm{nm}$ for Co and $\mathrm{Ni}[139,142,143])$ can lead to additional effects in uncorrected spectra. Enhanced orbital moments in clusters are mainly located in the two outermost layer (cf. Fig. 21 in Section 5.1), which are probed with a higher probability than the inner layer having lower orbital moments due to a higher number of nearest-neighbours. Thus, TEY overestimates the average orbital moment of the cluster. However, even in the case of very large nanoparticles with about $12 \mathrm{~nm}$, XMCD still probes $70 \%$ of the cluster volume. Because both effects mentioned here are of opposite sign, we assume that the effective deviation should be in the order of $10-15 \%$ for the orbital moment and much smaller for the spin moment in the case of $12 \mathrm{~nm}$ Fe clusters. This is in agreement with extended Monte Carlo simulations [144].

\subsubsection{X-ray magneto-optical Kerr effect}

With the availability of highly brilliant and tuneable synchrotron radiation sources MOKE was successfully extended from the visible light to the soft X-ray regime, where strongly enhanced effects at the element-specific core level resonances were observed (see [145,146] and references therein). Near the $2 p$ edges of the $3 d$ metals Kao et al. [147] found large resonantly enhanced differences in specularly reflected p-polarized radiation when reversing the magnetization state of the sample perpendicularly to the plane of incidence. In contrast to the similar transverse magneto-optical Kerr effect (T-MOKE) using visible light, T-MOKE in the soft X-ray regime has the advantage of strongly enhanced magnitude and element-specificity. Therefore, it is well suited for studying the magnetic reversal of combined $3 \mathrm{~d}$ metal samples. We will briefly demonstrate this technique at large Fe clusters deposited onto a well-ordered

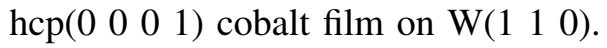

A typical set-up is shown in the inset of Fig. 19d. The magnetic field is applied perpendicularly to the plane of incidence and the sample is rotatable around its azimuth. The angle of incidence should be chosen as a compromise between reflectivity (rapidly dropping when the angle is increased) and the magnetic sensitivity in the T-MOKE (large around $45^{\circ}$ ). Here, a grazing angle of $22^{\circ}$ has been chosen. 


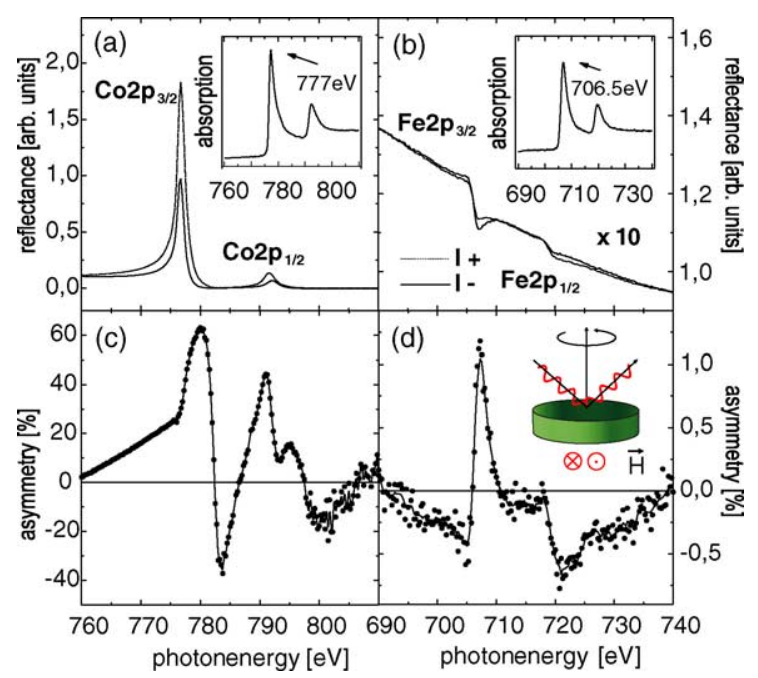

Fig. 19. Soft X-ray T-MOKE data obtained from $12 \mathrm{~nm}$ Fe clusters (right part) on top of a magnetized cobalt layer (left part) on W(l 110$)$. Upper panels: reflectance spectra $I+$ and $I-$ for opposite magnetization directions; lower panels: corresponding asymmetry curves. The insets show the respective absorption edges of Co and Fe and the experimental geometry.

The reflectance spectra taken from the Co film in Fig. 19a show a resonantly enhanced reflectivity with huge intensity differences in the vicinity of the $2 p$ levels when reversing the magnetization. The corresponding asymmetry with values up to $60 \%$ is shown in the lower left part. The shape of soft X-ray reflectance spectra and related asymmetries, respectively, is not only determined by electronic resonances but also by interference depending sensitively on the experimental geometry and sample morphology [146,148,149]. Thus, the reflectivity signals in general deviate from the respective absorption spectra shown in the insets in the upper panels. Fig. 19b shows reflectance spectra in the range of the $2 p$ edges of the iron clusters scaled in order to show the magnetic effect at the much lower Fe $2 \mathrm{p}$ edges at 706.5 and $720 \mathrm{eV}$, respectively. The decrease of the reflected intensity from iron clusters with respect to the cobalt films is caused by the smaller amount of deposited material and by diffuse light scattering of the clusters with a size of about $12 \mathrm{~nm}$ already being close to the incident wavelength of about $2 \mathrm{~nm}$. The related asymmetry taken from the Fe clusters is displayed in Fig. 19d. In contrast to the strong effects of the Co film, only weak ones are observed. The small values for the asymmetries (up to $1.0 \%$ ) are due to the comparably large background of non-resonantly reflected radiation from cobalt and tungsten, respectively. But obviously the resonantly enhanced effects near the Fe $2 p$ edges are significantly stronger than other possible non-resonant magneto-optical contributions of the Co film [150] and enable investigations at randomly distributed Fe clusters.

\section{Magnetic moments of supported 3d transition metal clusters}

This section focuses on exposed mass-filtered Fe and Co clusters of different size regimes being in contact with a surface. Especially for $3 \mathrm{~d}$ metal clusters smaller than about 500 atoms calculations $[34,39,151]$ predict both an increased spin moment and an enhanced orbital moment. The fundamental 
building block in the case of cluster-assembled materials is not the free cluster but the isolated cluster supported on a substrate or embedded in a matrix. Magnetic measurements in free clusters are restricted to gradient-field deflection experiments that determine the total magnetic moment. Orbital and spin moments can be determined in clusters deposited on a substrate by techniques such as XMCD (Section 4.3.1). The first part is related to small Fe clusters with diameters of about $2 \mathrm{~nm}$ deposited on HOPG (and capped Fe cluster in Section 5.3), whereas the second subsection deals with large Fe clusters (6-12 nm). Experimental studies on deposited magnetic clusters emphasize the importance of extending our present theoretical understanding on free clusters to situations where the clusters are in contact with a macroscopic environment. A systematic comparison between the properties of free and deposited particles appears therefore as a fundamental step towards the characterization of the specific properties of these nanostructured materials. This is carried out in the last subsection for the case of small Co clusters on $\operatorname{Pd}\left(\begin{array}{lll}1 & 1 & 1\end{array}\right)$.

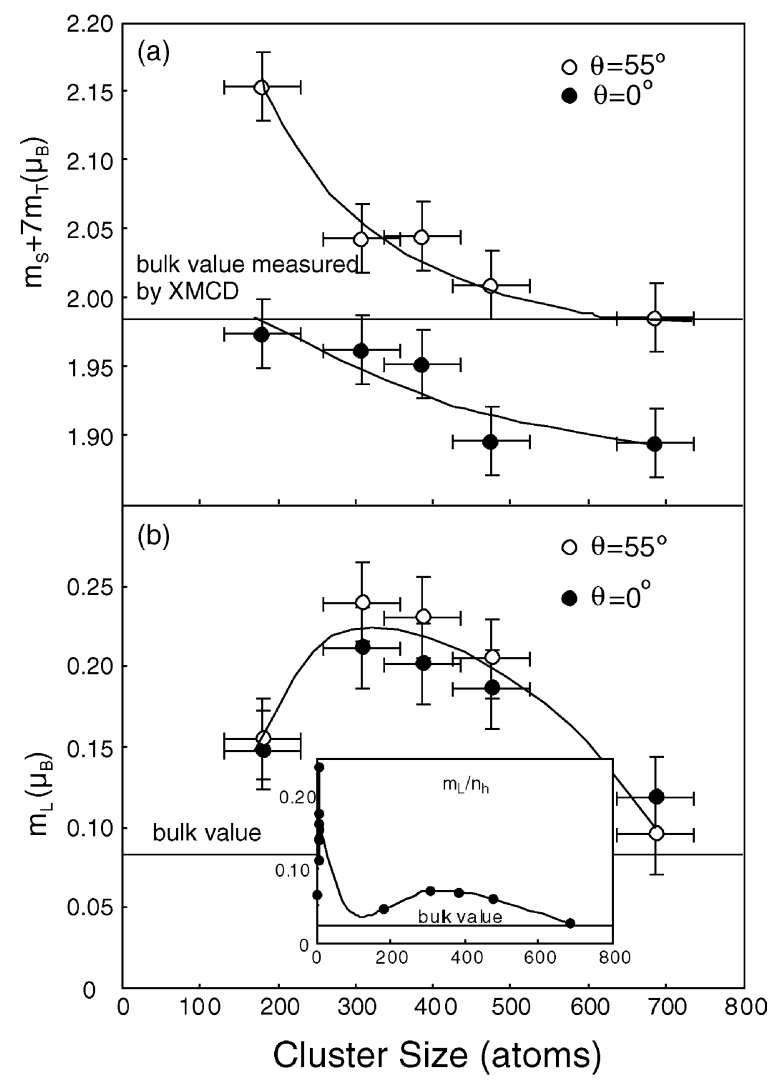

Fig. 20. (a) $m_{\mathrm{S}}+7 m_{\mathrm{T}}$ values obtained by XMCD from isolated Fe clusters on HOPG as a function of size. Measurements at $\theta=0^{\circ}$ (filled circles) and $55^{\circ}$ (open circles) are displayed and $m_{\mathrm{T}}=0$ in the data at $\theta=55^{\circ}$ (the 'magic angle') revealing the pure spin moment. The measured bulk value was from a $250 \AA$ thick conventional Fe film. (b) $m_{\mathrm{L}}$ values obtained by XMCD showing a peak at $\approx 300$ atoms. There is no observable difference between the measurements at $\theta=0^{\circ}$ (filled circles) and $55^{\circ}$ (open circles) indicating that the orbital moment is isotropic. The inset compares the present data with the measurements of Lau et al. [114] for very small Fe clusters $(n=2-9)$ on Ni substrates. The combined data reveals a remarkable change in $m_{\mathrm{L}}$ with cluster size [152]. 


\subsection{Spin and orbital moments in small mass-selected Fe clusters}

Fig. 20 shows the spin and orbital moments in mass-selected Fe clusters for sizes below 700 atoms deposited in situ onto HOPG substrates held at $6 \mathrm{~K}$ to a coverage of $\approx 0.03$ cluster monolayers [152]. The spin $m_{\mathrm{S}}+7 m_{\mathrm{T}}$ values are shown for data taken at normal incidence $\left(\theta=0^{\circ}\right)$ and with the sample rotated to the 'magic angle' of $55^{\circ}$ incidence. As mentioned in Section 4.3.1, the contribution from the magnetic dipole term $m_{\mathrm{T}}$ contribution vanishes at this angle revealing the pure spin moment. The spin moment shown in Fig. 20a increases with decreasing cluster size and is enhanced by $10 \%$ relative to the bulk value at the smallest size measured (181 atoms). Note how the data taken at $55^{\circ}$ and normal incidence diverge revealing the increasing importance of the dipole contribution as the cluster size decreases. The bulk value for comparison was obtained by XMCD from a $250 \AA$ thick conventional Fe film deposited in situ. The orbital moment shown in Fig. 20b also increases with decreasing cluster size and is enhanced by a factor of 3 relative to the bulk value for cluster sizes around 300 atoms. In the smallest clusters however it diminishes again, which may be due to the formation of a closed shell system, for example, the 147-atom icosahedron. The inset in Fig. 20b compares the measurement of $m_{\mathrm{L}} / n_{\mathrm{h}}$ from Fe clusters on HOPG obtained by Baker et al. [152] with those of Lau et al. [114] for very small Fe clusters in the size range 29 atoms deposited on Ni substrates. Thus, the orbital moments of Fe clusters shown in the lower part of Fig. 20 support the strong influence of the particle geometry (especially open and closed shells) on the orbital moment. The size-dependence of the orbital moments in clusters has already been discussed in Section 3.5 for nickel clusters. In the following we will focus on the orbital moment of each individual shell in a cluster for the example of a fcc Ni cluster containing up to 164 atoms [34]. Here, the orbital moments for the most stable cluster structures have been determined for each shell. The results show oscillations in the value of $m_{\mathrm{L}}$ with the number of atoms in the cluster. In addition, as shown in Fig. 21, it has been discovered that in larger clusters the enhanced orbital moment is localized in the outer two

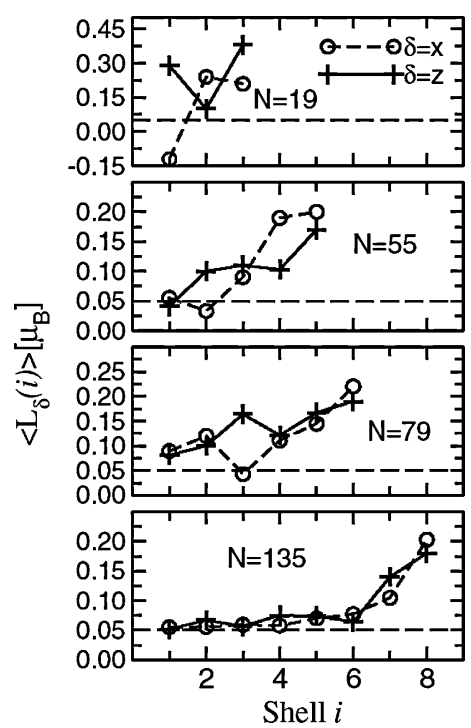

Fig. 21. Local orbital moments along the spin magnetization direction $\delta$ in fcc-like Ni clusters average at each NN shell $i$ surrounding the central atom $i=1$ [34]. 


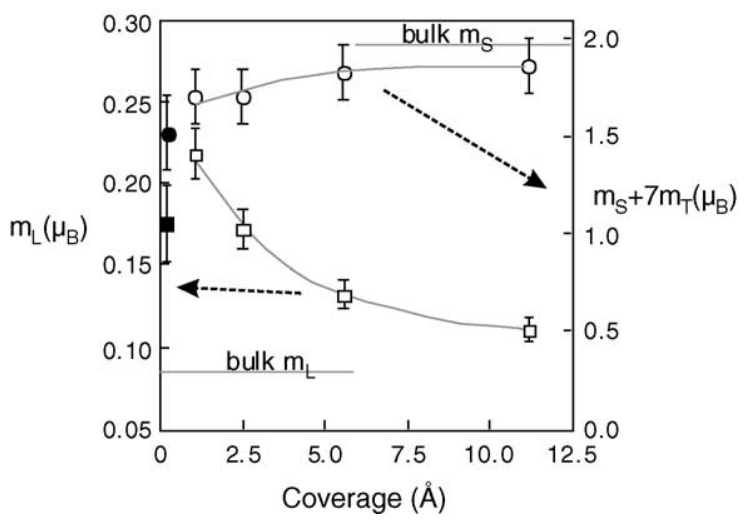

Fig. 22. Coverage dependence of the orbital moments $m_{L}$ (squares, see left scale) and spin moments $m_{\mathrm{s}}+7 m_{\mathrm{T}}$ (circles, see right scale) for Fe clusters on HOPG [112].

atomic shells of the cluster with the orbital moment in the interior having a value close to the bulk. This result will be important for the discussion of large Fe clusters (up to 100,000 atoms) in Section 5.2.

Fig. 22 shows the orbital and spin moments of unfiltered Fe clusters deposited on HOPG as a function of coverage, from isolated clusters to the $2 \mathrm{D}$ percolation threshold. The open symbols show the data for unfiltered deposits with a cluster size distribution peaking at 400 atoms. The closed symbols are the values obtained from a low coverage mass-selected deposit with the filter set to the peak of the size distribution (400 atoms). The orbital moment at low coverages shows a factor of 3 enhancement relative to the bulk value but decays towards the bulk value as the clusters come into contact. This efficient quenching indicates partial coalescence at the Fe-Fe interfaces. The XMCD data displayed in Fig. 22 were taken at normal incidence so the spin moment has a non-vanishing dipole contribution. The small rise in the spin term is most likely attributed to a decrease in the dipole moment (opposite to the spin) as the clusters come into contact [112]. The total magnetic moments in the exposed Fe clusters are shown in Fig. 23 together with gradient-field deflection measurements in free Fe clusters. It is evident that the

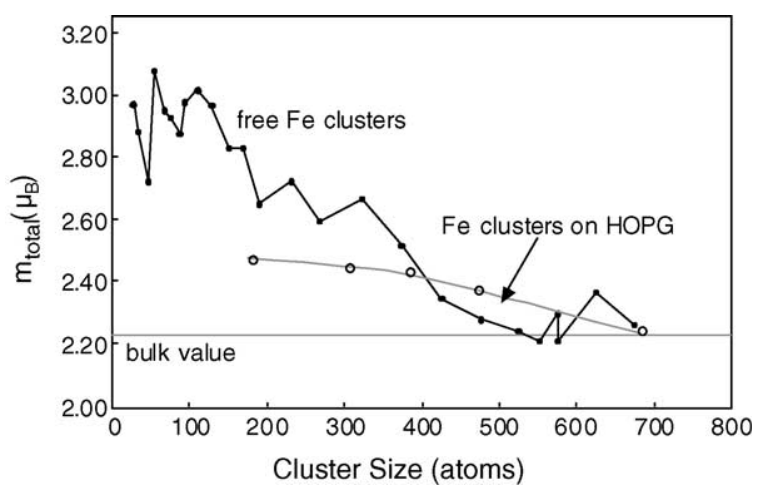

Fig. 23. Total magnetic moment $m_{\text {total }}$ in isolated Fe clusters on HOPG as a function of size (open circles) [152] in comparison to the total moments in free Fe clusters (filled circles, taken from [20]). It should be mentioned that the XMCD data published in [152] have additionally been corrected as outlined in Section 4.3.1. 
supported clusters have a significantly enhanced moment relative to the bulk (about half of which comes from the increased orbital moment). The total moment is smaller than in the free clusters.

\subsection{Spin and orbital moments in large mass-filtered clusters}

The results for exposed Fe clusters from the previous chapter open the question to what extent of particle size deviations from the bulk magnetic properties are observable. Total magnetic moments measured on free Fe clusters as well as on exposed Fe clusters on HOPG show a vanishing influence of the cluster size and thus a bulk-like behaviour in clusters above 600-700 atoms. In the following, we will present experimental results on large exposed Fe clusters in the size regime up to $12 \mathrm{~nm}$ or roughly 100,000 atoms per cluster, respectively. Such nanoparticles can be created by means of the arc cluster ion source ACIS (see Section 2.2) under UHV conditions. In contrast to the experiments in Section 5.1, the mass-filtered Fe clusters have predominantly been deposited onto epitaxially ordered $\operatorname{Co}\left(\begin{array}{llll}0 & 0 & 0 & 1\end{array}\right)$ films on W(l 110$)$ with a thickness of about $3 \mathrm{~nm}$. The films are atomically flat and possess well-defined magnetic properties [153-156]. Deposition of clusters onto ferromagnetic substrates is an alternative approach to obtain even at room temperature magnetically saturated particles that are necessary for XMCD investigations. Lau et al. [114] have used this method to investigate very small mass-selected Fe clusters on a $\mathrm{Ni}$ film on $\mathrm{Cu}\left(\begin{array}{lll}1 & 0 & 0\end{array}\right)$, however, the measurements were carried out at very low temperatures. Generally, magnetic measurements on clusters deposited on ferromagnetic layer systems require investigation methods with a chemical sensitivity (cf. Section 4.3).

In order to examine the magnetic interaction between the Fe clusters and the Co film, T-MOKE experiments have been performed in the soft X-ray regime, cf. reflectivity spectra displayed in Fig. 19. The asymmetries near the $2 p$ edges enable us to record element-specific hysteresis loops from the cobalt film and the $12 \mathrm{~nm}$ Fe clusters separately. Angular dependent results obtained at photon energies of

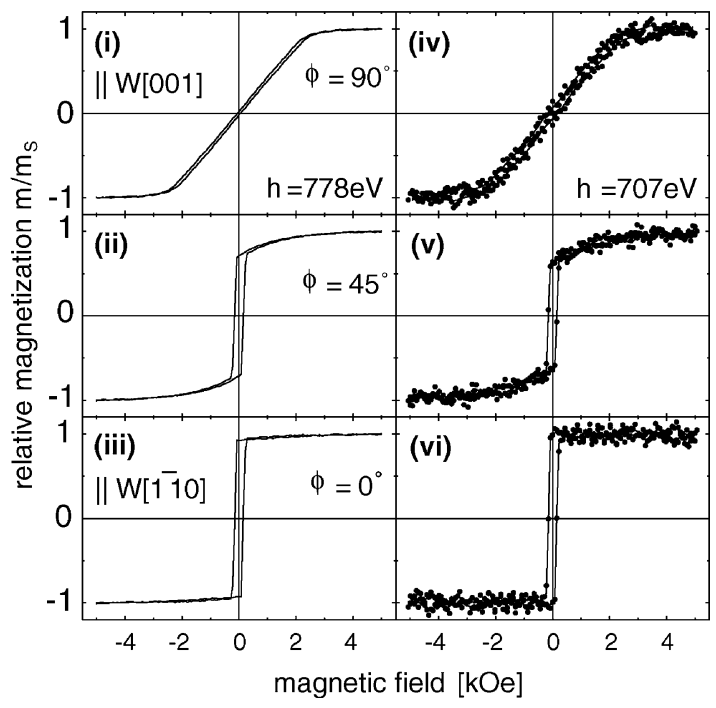

Fig. 24. Hysteresis loops taken from iron clusters on top of a cobalt film at the respective 2p core levels of Co (left) and Fe (right part). The angles $\phi$ refer to azimuthal rotation with respect to the surface normal, cf. inset in Fig. 19 with $\phi=0^{\circ}$ being the easy magnetization axis and $\phi=90^{\circ}$ the hard magnetization axis [37]. 
$788 \mathrm{eV}$ (Co, left part) and $707 \mathrm{eV}$ (Fe, right part) are shown in Fig. 24. The topmost curves (i) and (iv) were recorded along the hard magnetic axis of Co(l $\left.\begin{array}{llll}0 & 0 & 1\end{array}\right)$ films on W(lllll 110$)$ with the W[ $\left.\begin{array}{lll}0 & 0 & 1\end{array}\right]$ direction parallel to the magnetic field. From the top to the bottom the sample orientation was rotated in two steps to the magnetic easy axis of the Co film. The Co magnetization curves confirm the well-known uniaxial in-plane anisotropy in these films. The hysteresis loops of the iron clusters follow the curves of the cobalt film closely suggesting a strong interaction between the Fe clusters and the Co film. In particular, switching the film along the easy axis provides a remanent saturation magnetization required for XMCD studies without external magnetic fields.

XMCD investigations are carried out in order to determine the orbital $\left(m_{\mathrm{L}}\right)$ and spin moments $\left(m_{\mathrm{S}}\right)$ of

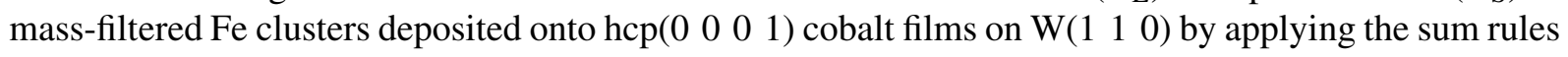
outlined in Section 4.3.1. Fig. 25 displays in the upper panels photoabsorption spectra taken with circularly polarized X-ray radiation at the respective $2 \mathrm{p}_{3 / 2}$ and $2 \mathrm{p}_{1 / 2}$ core levels at about $h v=778$ and $793 \mathrm{eV}$ for the cobalt layer on W(l 10 ) and for the deposited Fe clusters ( 708 and $721 \mathrm{eV})$. The angle of incidence is $60^{\circ}$ with respect to the surface normal (i.e. close to the 'magic angle') and thus the dipole term $\left\langle T_{z}\right\rangle$ entering Eq. (18) can be neglected. The easy magnetization axis of the Co film has been chosen parallel to the magnetization coil; thus, all experiments could be carried out in remanence. The lower parts show the corresponding dichroism signals used for the determination of the magnetic moments. Assuming that the magnetic spin moment of nanoparticles in this size regime has already reached the bulk value, the discussion will focus on the ratio of the orbital to the spin moment $\left(m_{\mathrm{L}} / m_{\mathrm{S}}\right)$. Additionally, this procedure avoids any assumption on the number of holes $n_{\mathrm{h}}$ in the valence band or the degree of polarization, cf. Eqs. (17) and (18) in Section 4.3.1.

The evaluation of the data in Fig. (25) reveals a ratio of $m_{\mathrm{L}} / m_{\mathrm{S}}=0.07 \pm 0.01$, clearly enhanced when compared to the respective bulk value of 0.043 [138]. A more systematic overview on the size dependence of $m_{\mathrm{L}} / m_{\mathrm{S}}$ is given in Fig. (26), where the results from Section 5.1 (exposed Fe clusters on HOPG up to $2.3 \mathrm{~nm}$ ) are combined with data for Fe clusters between 6 and $12 \mathrm{~nm}$. In the left part, this figure also includes a data point from very small Fe clusters on $\mathrm{Ni} / \mathrm{Cu}\left(\begin{array}{lll}1 & 0 & 0\end{array}\right)$. It should be mentioned that

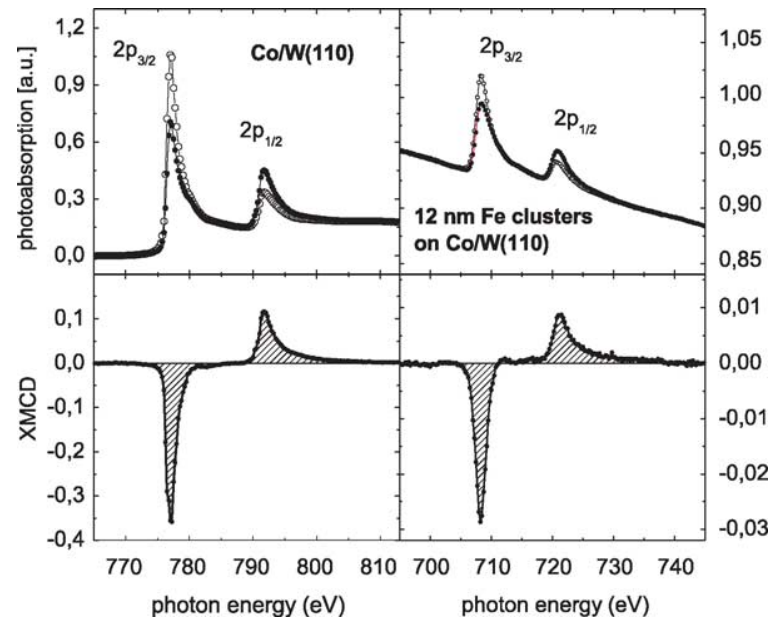

Fig. 25. Photoabsorption spectra taken with circularly polarized radiation from Fe clusters (right part) on an hcp( 00001$)$ cobalt film (left part) on W(1 100$)$ for opposite magnetization directions (open and solid dots). The lower panels show the intensity difference, i.e. the XMCD signal [37]. 


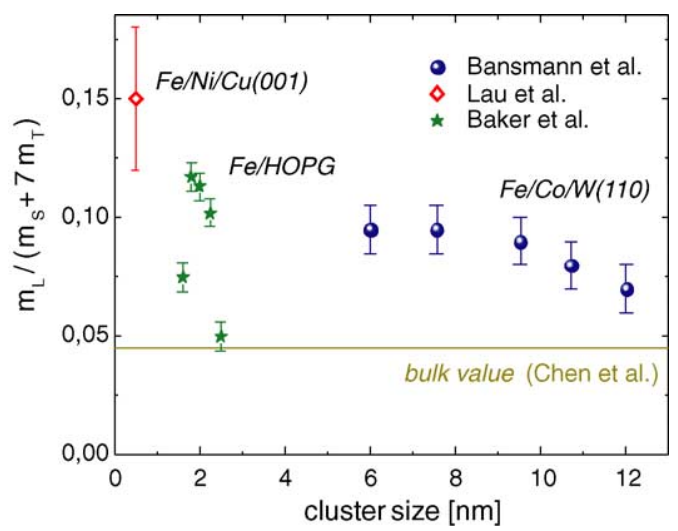

Fig. 26. Ratio of orbital $\left(m_{\mathrm{L}}\right)$ to spin moment $\left(m_{\mathrm{S}}+7 m_{\mathrm{T}}\right)$ for Fe clusters on surfaces: Fe clusters on Ni/Cu(1 00$)$ (from [114]) (left part), Fe clusters on HOPG (from [152]) (middle), and large Fe clusters on Co/W(1 10$)$ (from [37]) (right part). The bulk value (solid line) has been taken from [138].

the results from Fe clusters with 1-9 atoms have been averaged, for a detailed discussion we refer to the original publication in [114]. The experimental data for large Fe clusters (filled circle) exhibit $m_{\mathrm{L}} / m_{\mathrm{S}}$ values from 0.07 at a cluster size of $12 \mathrm{~nm}$ up to 0.095 for Fe clusters with $6 \mathrm{~nm}$. All these values are clearly above the corresponding bulk value of 0.043 . The enhanced ratio is related to an increase of the orbital moment in the outer two shells which exhibit a large number of surface atoms compared to the total number of atoms in the cluster. When assuming a spherical shape the ratio of surface to volume atoms amounts to $43 \%$ in case of a $6 \mathrm{~nm}$ Fe cluster and still 23\% for Fe clusters with a size of $12 \mathrm{~nm}$. However, in the larger cluster size regime, one has to bear in mind that the determination of spin and orbital moments in total electron yield gives rise to systematic deviations. Relevant systematic errors in XMCD measurements are briefly described in Section 4.3.1. Here, the uncertainties are included in the error bars in Fig. 26.

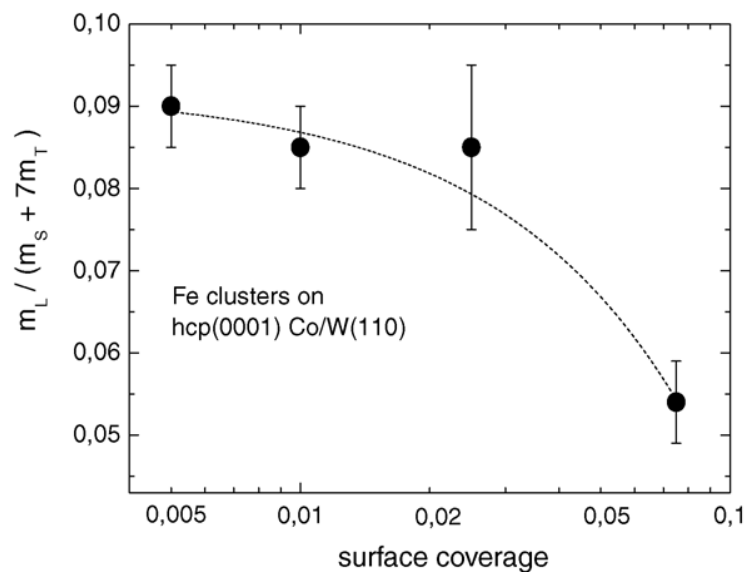

Fig. 27. Ratio of orbital $\left(m_{\mathrm{L}}\right)$ to spin moment $\left(m_{\mathrm{S}}+7 m_{\mathrm{T}}\right)$ of $9 \mathrm{~nm} \mathrm{Fe}$ clusters on Co/W(1 10$)$ as a function of the surface coverage [37]. The $x$-axis denotes the area on the surface covered by Fe clusters when assuming the mean size from TEM measurements (cf. Fig. 5). The dotted line serves to guide the eye. 


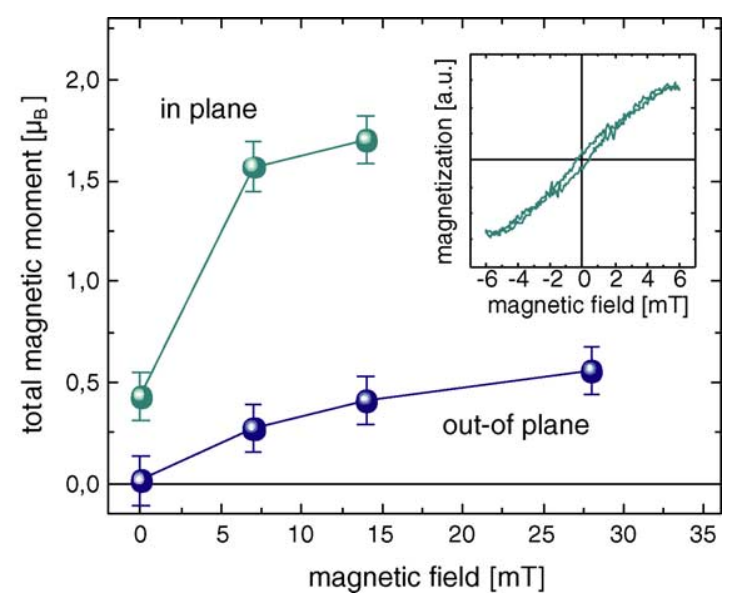

Fig. 28. Total magnetic moment of $12 \mathrm{~nm}$ Fe clusters on W(1 110$)$ plotted for in-plane and out-of-plane magnetization. The inset displays a hysteresis curve taken with an in-plane magnetization [159].

Analogous to the experiments at smaller Fe clusters (cf. Fig. 22) also the coverage dependence of larger Fe clusters has been investigated. In Fig. 27, the ratio of orbital to spin moment $m_{\mathrm{L}} / m_{\mathrm{S}}+7 m_{\mathrm{T}}$ for $9 \mathrm{~nm}$ Fe clusters is displayed as a function of the surface coverage. Under the assumption that the spin moment is bulk-like, we find that the percolation of individual particles reduces the orbital moment similar to smaller Fe clusters (see Fig. 22). At a coverage of less than one monolayer, the orbital moment nearly reaches the bulk value of iron $\left(m_{\mathrm{L}} /\left(m_{\mathrm{S}}+7 m_{\mathrm{T}}\right)=0.043\right)$.

Up to now the large Fe clusters have always been deposited onto ferromagnetic Co(l $\left.\begin{array}{llll}0 & 0 & 0 & 1\end{array}\right)$ films on $\mathrm{W}\left(\begin{array}{lll}1 & 1 & 0\end{array}\right)$ where the clusters feel the presence of a strong exchange field inducing an in-plane magnetic anisotropy in the clusters. Now we will concentrate on the magnetic behaviour of Fe clusters on a nonmagnetic support. Therefore, $12 \mathrm{~nm}$ Fe clusters have been deposited onto a clean W(lll 110$)$ surface and variable external magnetic field have been applied in-plane (i.e. along the W[1 1,0$]$ axis) and out-ofplane to the sample (see Fig. 28 during the XMCD investigations). The angle of incidence $\left(60^{\circ}\right)$ has always been kept constant. The measured total magnetic moments (i.e. the sum of orbital and spin moment) are the projections of the real magnetic moments on the direction of the magnetic field. Clearly, the clusters can be much more easily magnetized in the surface plane than perpendicular to it. Although a magnetic saturation could not be reached with such small magnetic fields, the in- plane magnetization nevertheless shows a total magnetic moment of about $1.7 \mu_{\mathrm{B}}$ at $14 \mathrm{mT}$. Additionally, the inset shows an in-plane hysteresis loop confirming a small but finite remanence that could not be observed in an out-ofplane magnetization. This pronounced in-plane anisotropy can be explained by two effects which both favour an in-plane magnetization: (i) a strong surface anisotropy at the $\mathrm{Fe}-\mathrm{W}$ interface as known from $\mathrm{Fe}\left(\begin{array}{lll}1 & 1 & 0\end{array}\right)$ films on W(lll 110$)\left[\begin{array}{ll}157,158\end{array}\right]$ and (ii) due to a wetting of the tungsten surface which leads to an oblate cluster shape.

\subsection{Fe particles coated with cobalt}

Edmonds et al. [36] investigated the effect of coating assemblies of exposed Fe cluster films on HOPG with Co films in situ and they showed that the combined film consisted of Co islands with embedded Fe 


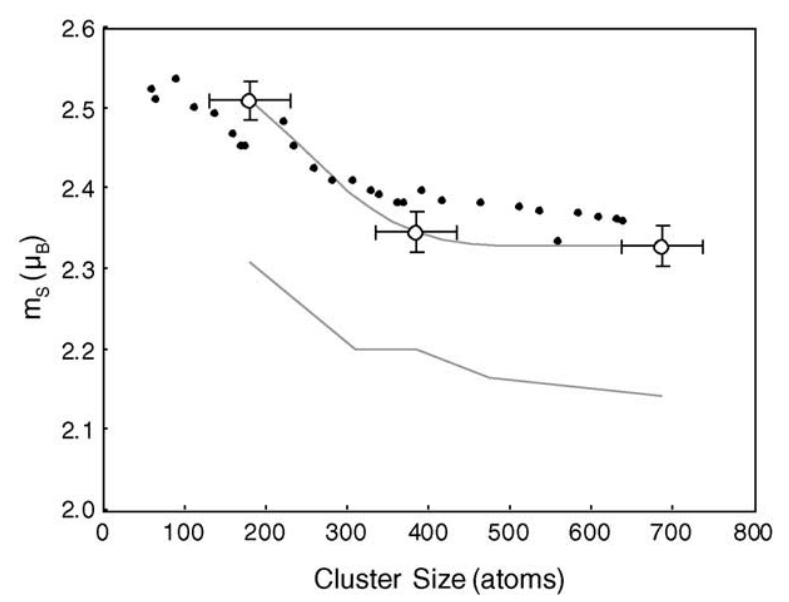

Fig. 29. Spin moment measured by XMCD in Co-coated Fe clusters on HOPG as a function of the Fe cluster size (open circles, from [152]) compared with the calculated spin moment in free Co-coated $\mathrm{Fe}$ clusters with composition $\mathrm{Fe}_{N} \mathrm{Co}_{1021-N}$ as a function of $N$ [39] (filled circles). The lower grey line shows the measured spin moments in the exposed clusters prior to coating [152]. Please note, that the experimental values from [152] have been corrected analogously to Fig. 23.

clusters. The Fe clusters were exchange-coupled to the Co film and the magnetization showed a strong inplane shape anisotropy due to the Co islands. The fundamental orbital and spin moments localized in the Fe clusters could be extracted due to the chemical specificity of XMCD. Fig. 29 shows the measured sizedependent spin moment, after correcting for the systematic error discussed earlier, compared with the calculation by Xie and Blackman [39] for free Co-coated Fe clusters, with a composition of $\mathrm{Fe}_{N} \mathrm{Co}_{1021-N}$. The agreement is good and demonstrates an increase in the spin moment of $\approx 0.2 \mu_{\mathrm{B}}$ relative to the exposed clusters. Over a significant fraction of the size range the total magnetic moment per atom measured in the supported Co-coated Fe clusters is as large as in free Fe clusters.

The theoretical calculations were done using tight binding (TB) methodology with terms that are dependent on the local environment [39,160-162]. The TB parameters are obtained by fitting to the results of ab initio calculations performed on small clusters. A model is constructed of atoms in shells around a central site in a bcc structure with the theoretical lattice constant of Fe. The clusters are constructed layer by layer along the $\left(\begin{array}{lll}1 & 0 & 0\end{array}\right)$ and $\left(\begin{array}{lll}1 & 1 & 0\end{array}\right)$ directions of the bcc structures. The resulting main shell-closed clusters are covered with $6\left(\begin{array}{lll}1 & 0 & 0\end{array}\right)$ faces and $12\left(\begin{array}{lll}1 & 1 & 0\end{array}\right)$ faces. Sub-shells are filled with successive $\mathrm{O}_{\mathrm{h}}$ symmetry occupation of free sites. Previous calculations of iron clusters [163,164] assumed a spherical growth pattern. We find from our TB calculations that the total energy of regularly shaped Fe clusters generated as described are lower than those of the same size spherically shaped clusters. In addition to free $\mathrm{Fe}_{N}$ clusters, we also study $\mathrm{Fe}_{N}$ clusters coated with layers of $\mathrm{Cu}$ or $\mathrm{Co}$ atoms. For the coated clusters, the $\mathrm{Fe}$ cores are covered by at least one layer of $\mathrm{Cu}$ or $\mathrm{Co}$ ensuring that the nearest-neighbour coordination of all Fe atom is the same as in bcc bulk Fe. Using symmetry-adapted basis functions, the Hamiltonian matrix is easily diagonalized for systems containing more than 1000 atoms. The results for the averaged local magnetic moment per iron atom are displayed in Fig. 30. Plots are shown for free and for $\mathrm{Cu}$ and $\mathrm{Co}$ covered iron clusters. Two plots for Co-coated $\mathrm{Fe}$ clusters are shown in Fig. 30. $\mathrm{Fe}_{N} \mathrm{Co}_{1021-N}$-I clusters have the $N \mathrm{Fe}$ atoms filling inner sub-shells with the $\mathrm{Co}$ atoms in the outer ones. $\mathrm{A} \mathrm{Fe}_{N} \mathrm{Co}_{1021-N^{-}}$II cluster is constructed from a type $i$ one by replacing all $\mathrm{Fe}$ atoms in a sub-shell in the interface region with Co atoms. Such a cluster will give an indication of 


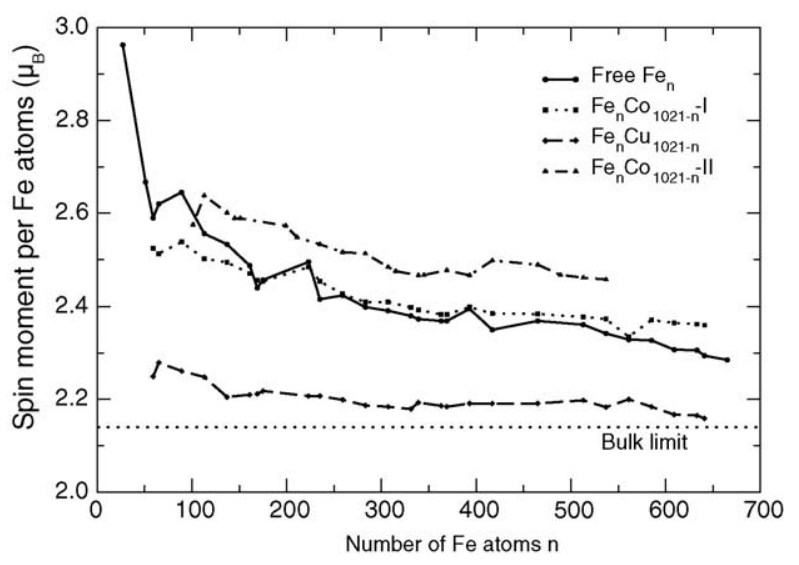

Fig. 30. Average local spin moment of $\mathrm{Fe}$ atoms in clusters of size $N$. Plots are: free $\mathrm{Fe}$ clusters (free $\mathrm{Fe}_{N}$ ), $\mathrm{Cu}$-coated $\mathrm{Fe}$ clusters $\left(\mathrm{Fe}_{N} \mathrm{Co}_{1021-N}\right)$, Co-coated Fe clusters $\left(\mathrm{Fe}_{N} \mathrm{Co}_{1021-N}-\mathrm{I}\right)$, Co-coated Fe clusters with intermixing at interface $\left(\mathrm{Fe}_{N} \mathrm{Co}_{1021-N}\right.$-II $)$ [39].

possible effects arising from the diffusion of Co atoms across the interface. It is well known [10,20] that the magnetic moments of free iron clusters are enhanced compared to the bulk materials, and that the average magnetization per atom decreases non-monotonically to the bulk value with increasing cluster size. The calculated averaged magnetic moments for free clusters shown in Fig. 30 correctly reproduce the experimental trend of a convergence to the bulk value with some small oscillation. The enhanced moment is largely due to the reduced nearest-neighbour coordination of the surface atoms, and it is to be expected that the enhancement will be largely cancelled out when the cluster is coated by additional layers of atoms. This is indeed what is found for the $\mathrm{Cu}$-coated clusters where the magnetization is reduced to marginally above the bulk value as is seen in Fig. 30. Similar behaviour is seen experimentally when clusters are deposited onto a surface such as a noble metal or HOPG [36]. Coating the Fe core with $\mathrm{Co}$ instead of $\mathrm{Cu}$ produces very different behaviour as is shown in the $\mathrm{Fe}_{N} \mathrm{Co}_{1021-N^{-}} \mathrm{I}$ plot in Fig. 30. Despite the fact that the coordination of the surface Fe atoms is the same as that of the bulk, the average magnetic moment on the $\mathrm{Fe}$ is close to that of free iron clusters. It has been pointed out before that $\mathrm{Fe}$ is magnetically weak because of an insufficient electron-electron interaction to band-width ratio, while in $\mathrm{Fe}-\mathrm{Co}$ alloys this interaction for the Co atoms can increase the exchange splitting of the Fe $3 \mathrm{~d}$ bands $[93,165,166]$. The local magnetic moments for each sub-shell of $\mathrm{Fe}_{339}$ in different environments are depicted in Fig. 31. The results for other cluster sizes are similar to this one. Referring first to the free Fe cluster, the enhancement over the bulk moment comes mainly from the atoms on the surface of the cluster (there are 170 in number on sites labeled $\left(\begin{array}{lll}6 & 0 & 0\end{array}\right)$ to $\left(\begin{array}{lll}4 & 4\end{array}\right)$ ). The coordination numbers of the surface atoms are noted on the figure. A coating of $\mathrm{Cu}$, restores the bulk coordination number, and yields a magnetization that is close to that of bulk. With Co atoms as neighbours, however, the reduction of the moments of the $\mathrm{Fe}$ atoms at the interface is much less. The Co neighbours provide an effective enhancement to the exchange splitting of the Fe that virtually compensates for the band-width increase that occurs because of their presence. The element-specific nature of the XMCD measurements provides an experimental probe of the average moment on the Fe core [33,36]. It can be seen from Fig. 29 that the theoretical results for $\mathrm{Fe}$ embedded in $\mathrm{Co}$ are in excellent agreement with the experimental observations for 200-700 Fe atom clusters. For Fe clusters embedded in a Co film, XMCD data show that the number 


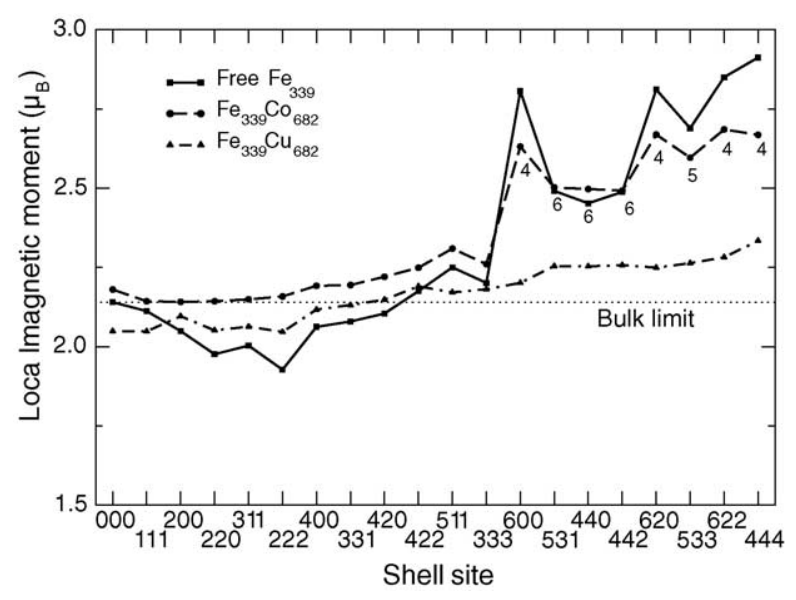

Fig. 31. Local moment of $\mathrm{Fe}$ atoms for free and for $\mathrm{Cu}$ or $\mathrm{Co}$-coated $\mathrm{Fe}_{339}$ clusters as a function to positions on bcc lattice (in units of one-half the lattice constant). The figures on the plot are the numbers of Fe neighbours of surface (or interface) Fe atoms [39].

of Fe $3 \mathrm{~d}$ holes per atom is indistinguishable from the bulk demonstrating that the grains consist mainly of pure Fe with intermixing confined to the particle surfaces [36]. However, Fe and Co are intermiscible, and this may result in some Co atoms diffusing through the interface region into the Fe particle. To model the effect of this diffusion, we replace some of the Fe atoms in Co-coated Fe clusters by Co atoms. For ease of calculation, we maintain cluster symmetry by replacing all the atoms in one sub-shell. It can be seen from plot $\mathrm{Fe}_{N} \mathrm{Co}_{1021-N}$-II of Fig. 30 that this substitution produces a distinct enhancement in the average local moment on the Fe compared with free Fe clusters for sizes larger than 100 atoms. In order to see where the enhancement comes from, we show the local moment of different shell sites on a typical cluster in Fig. 32. The cluster $\mathrm{Fe}_{315} \mathrm{Co}_{706}$-II is constructed by replacing the $24 \mathrm{Fe}$ atoms in the $\left(\begin{array}{lll}5 & 1 & 1\end{array}\right)$ sub-shell of $\mathrm{Fe}_{339} \mathrm{Co}_{682}$-I with $\mathrm{Co}$ atoms. The increased moment comes largely from five shells (4 00 ), (4 20 ),

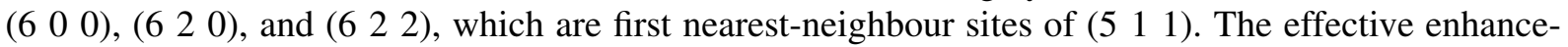

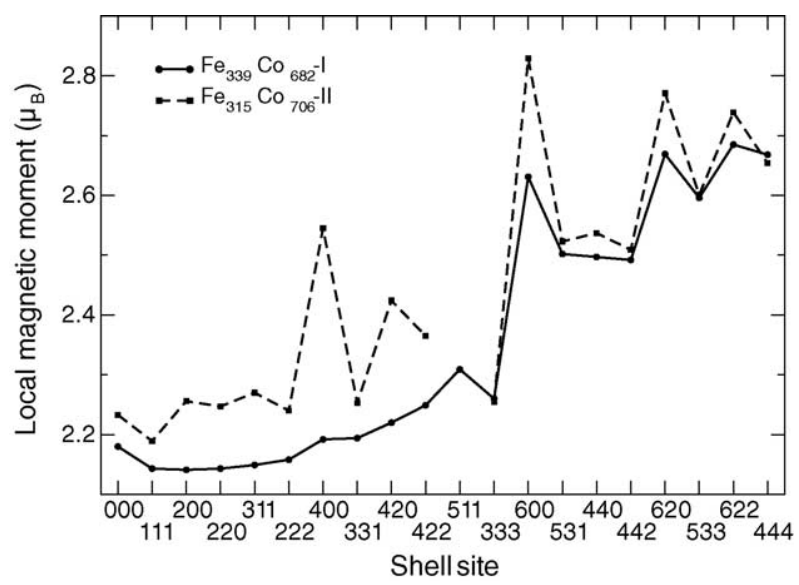

Fig. 32. Local moments of Co-coated Fe clusters. $\mathrm{Fe}_{315} \mathrm{Co}_{706}-\mathrm{II}$ is constructed from $\mathrm{Fe}_{339} \mathrm{Co}_{682}-\mathrm{I}$ by replacing $24 \mathrm{Fe}$ atoms at (5 11 1) sites with Co atoms [39]. 
ment to the exchange splitting on Fe sites produced by Co neighbours operates not only on atoms on the surface of the Fe core but also in the core region itself. The sensitivity of the magnetic moments of Fe to the local environment has also been observed in a recent study of Fe-Co alloys and multilayers [165].

\subsection{Ferromagnetic clusters on highly-polarizable substrates}

Spin and orbital moments as well as the magnetic anisotropy energy of Fe and Co clusters on noble metal substrates $[115,116,167,168]$ have been the subject of several theoretical studies. A variety of interesting magnetic behaviours has been revealed as a result of the competition between the reduction of local coordination numbers and the interactions with the substrate. The purpose of this section is to discuss the magnetic and electronic properties of ferromagnetic clusters on highly-polarizable TM substrates. $\mathrm{Co}_{N}$ clusters on $\mathrm{Pd}\left(\begin{array}{lll}1 & 1 & 1\end{array}\right)$ are expected to be particularly interesting due to the strong magnetic susceptibility of $\mathrm{Pd}$ and since the Co-Pd interfaces are known to show a remarkably rich magneto-anisotropic behaviour $[169,170]$. It is therefore very appealing, from both fundamental and technological standpoints, to investigate the possibility of tailoring the magnetic anisotropy of Co nanostructures on Pd in order to stabilize perpendicular magnetizations which could be useful in highdensity recording and memory devices $[11,171]$.

\subsubsection{Spin and orbital moments of $\mathrm{Co}_{N}$ on $\operatorname{Pd}\left(\begin{array}{lll}1 & 1 & 1\end{array}\right)$}

Table 2 summarizes results for the average magnetic moments per Co atom derived from selfconsistent tight-binding calculations on $\mathrm{Co}_{N}$ clusters deposited on the $\mathrm{Pd}\left(\begin{array}{lll}1 & 1 & 1\end{array}\right)$ surface. First of all, one observes that the total magnetic moments per Co atom $M_{\delta_{0}}$ of the deposited clusters are remarkably large for all the considered structures $(N \leq 13)$. The most important $M_{\delta_{0}}$ are found for the 1D monoatomic chains [structure (b)]. In this case, $M_{\delta_{0}}$ appears to depend very weakly on $N$ in the considered size range: $M_{x}=2.65,2.62$, and $2.61 \mu_{\mathrm{B}}$ for $N=3,7$, and 13, respectively. Moreover, the results for the 13-atom chain are actually not far from the infinite-length limit, for which $M_{x}=2.56 \mu_{\mathrm{B}}$ is obtained. Twodimensional-like clusters show somewhat smaller magnetic moments than the monoatomic chains. Here, $M_{\delta_{0}}$ decreases moderately with increasing $N$ as one approaches the $2 \mathrm{D}$ Co monolayer on $\operatorname{Pd}\left(\begin{array}{lll}1 & 1 & 1\end{array}\right)$ : $M_{y}=2.61 \mu_{\mathrm{B}}$ for the triangle, $M_{z}=2.49 \mu_{\mathrm{B}}$ for the rhombus, $M_{z}=2.40 \mu_{\mathrm{B}}$ for the hexagon, and $M_{x}=$ $2.32 \mu_{\mathrm{B}}$ for the infinite monolayer. Comparison with the results for free clusters shows that clustersubstrate interactions yield a significant enhancement of the magnetic moments per Co atom.

The average spin and orbital moments reported in Table 2 show that the magnetization $M_{\delta}=$ $\left(2\left\langle S_{\delta}\right\rangle+\left\langle L_{\delta}\right\rangle\right) / N$ of $\mathrm{Co}_{N}$ on $\operatorname{Pd}\left(\begin{array}{lll}1 & 1 & 1\end{array}\right)$ is mainly the result of three physically distinct effects. The first and leading contribution comes, as expected, from the spin moments at the Co atoms $i=1-N$ for which we obtain nearly saturated values $2\left\langle S_{z}\right\rangle_{\mathrm{Co}}=(1.66-1.69) \mu_{\mathrm{B}}$. These depend very weakly on size and structure, and are only slightly smaller than in the corresponding free clusters. The Co spin moments constitute about $63 \%$ of the total magnetization per Co atom in the monoatomic chains, and about 64$70 \%$ in the $2 \mathrm{D}$ islands $(N \leq 13)$.

The second important contribution to $M_{\delta}$ are the spin moments induced at the $\mathrm{Pd}$ atoms of the Co-Pd interface that amount to $(0.43-0.51) \mu_{\mathrm{B}}$, depending on the cluster size and structure. This corresponds to approximately $22 \%$ of the spin polarization per Co atom, and $19 \%$ of the total moment (see Table 2 ). The induced spin moments are responsible for the most part of the above discussed enhancement of $M_{\delta}$ in deposited clusters as compared to the free standing case. A more detailed analysis of the local magnetic moments at the Co-Pd interface, given in Section 5.4.3, allows to derive simple rules that relate the 
Table 2

Magnetic properties of $\mathrm{Co}_{N}$ clusters on $\operatorname{Pd}\left(\begin{array}{lll}1 & 1 & 1\end{array}\right)$

\begin{tabular}{|c|c|c|c|c|c|c|c|}
\hline$N$ & Structure & $2\left\langle S_{z}\right\rangle$ & $\left\langle L_{x}\right\rangle$ & $\left\langle L_{y}\right\rangle$ & $\left\langle L_{z}\right\rangle$ & $M_{\delta_{0}}$ & $\delta_{0}$ \\
\hline \multirow[t]{6}{*}{3} & (a) & 2.17 & 0.44 & 0.44 & 0.40 & 2.61 & $y$ \\
\hline & & (1.66) & (0.39) & $(0.39)$ & $(0.30)$ & (2.04) & $(y)$ \\
\hline & & [1.69] & {$[0.42]$} & {$[0.40]$} & {$[0.36]$} & [2.11] & {$[x]$} \\
\hline & (b) & 2.15 & 0.50 & 0.50 & 0.40 & $(2.65)$ & $x$ \\
\hline & & (1.66) & $(0.46)$ & $(0.42)$ & $(0.31)$ & $(2.12)$ & $(x)$ \\
\hline & & [1.69] & [1.36] & & {$[1.00]$} & [3.05] & {$[x]$} \\
\hline \multirow[t]{6}{*}{7} & (c) & 2.10 & 0.35 & & 0.30 & $(2.40)$ & $z$ \\
\hline & & (1.67) & $(0.28)$ & & $(0.24)$ & (1.91) & (z) \\
\hline & & [1.69] & {$[0.64]$} & & [0.29] & [2.33] & {$[x]$} \\
\hline & (b) & 2.16 & 0.46 & 0.45 & 0.38 & $(2.62)$ & $x$ \\
\hline & & (1.66) & $(0.43)$ & $(0.41)$ & $(0.30)$ & $(2.10)$ & $(x)$ \\
\hline & & [1.69] & {$[0.83]$} & & {$[0.63]$} & [2.52] & {$[x]$} \\
\hline \multirow[t]{6}{*}{13} & (d) & 2.09 & 0.34 & & 0.31 & $(2.40)$ & $z$ \\
\hline & & (1.66) & $(0.29)$ & & $(0.26)$ & (1.95) & $(z)$ \\
\hline & & [1.69] & {$[0.48]$} & & {$[0.35]$} & [2.17] & {$[x]$} \\
\hline & (b) & 2.15 & 0.46 & & 0.37 & $(2.61)$ & $x$ \\
\hline & & (1.66) & $(0.42)$ & & $(0.31)$ & (2.08) & $(x)$ \\
\hline & & [1.68] & {$[0.86]$} & & {$[0.56]$} & [2.54] & {$[x]$} \\
\hline
\end{tabular}

The averages per Co atom of the spin moment $2\left\langle S_{z}\right\rangle$, orbital moments $\left\langle L_{\delta}\right\rangle$ for $\delta=x, y$, and $z$, and total magnetic moment $M_{\delta_{0}}=2\left\langle S_{\delta_{0}}\right\rangle+\left\langle L_{\delta_{0}}\right\rangle$ for the easy axis $\delta_{0}$ are given in Bohr magnetons. The structures and magnetization directions are illustrated in Fig. 33. The results given in the first line (second line in parentheses) refer to deposited clusters including (excluding) the contributions of the $\mathrm{Pd}$ atoms at the $\mathrm{Co}-\mathrm{Pd}$ interface. The results in square brackets correspond to free $\mathrm{Co}_{N}$ having the same structure as the deposited clusters [151].

changes in the induced moments as a function of cluster size and geometry with the local environments of the Pd atoms.

The orbital magnetic moments $\left\langle L_{\delta}\right\rangle$ are the third main piece of the calculated magnetization. $\left\langle L_{\delta}\right\rangle$ is essentially parallel to the spin moment, as corresponds to elements having a d-shell which is more than half filled. Thus, they add to $\left\langle S_{\delta}\right\rangle$ representing about $15-19 \%$ of $M_{\delta}$. One observes that $\left\langle L_{\delta}\right\rangle$ is in general quite sensitive to the size and geometry of the cluster and to the direction $\delta$ of the magnetization. For example, 1D clusters usually develop larger orbital moments than 2D clusters of the same size, which in both cases tend to decrease with increasing $N$. Notice, moreover, that the anisotropy of $\left\langle L_{\delta}\right\rangle$ is far more important than the one of the spin moments. This anticipates a strong environment dependence of the MAE due the spin-orbit interactions. As in the case of the spin polarization, the largest part of the orbital moments comes from the Co atoms (values in parentheses). Still, the Pd contributions, (10-25)\% of $\left\langle L_{\delta}\right\rangle$ depending on the particular cluster and $\delta$, are not negligible (see Table 2). It is also interesting to remark that the average orbital moments at the Co atoms are significantly enhanced as compared to the corresponding bulk moment $\left\langle L_{\delta}\right\rangle($ Co-bulk $) \simeq 0.13 \mu_{\mathrm{B}}$. A similar effect has already been observed for Co films on Pd(1 1 1) [170], as well as in free and deposited TM clusters [34,115,116,168]. Quantitatively, the calculated Co orbital moments along the easy axis $\delta_{0}$ are $\left\langle L_{\delta_{0}}\right\rangle=(0.3-0.5) \mu_{\mathrm{B}}$, which corresponds to about $(0.15-0.2) \mu_{\mathrm{B}}$ per $\mathrm{d}$ hole, a value which is somewhat smaller, though not far, from the one observed in experiments on small Fe clusters deposited on $\mathrm{Ni}$ [114]. Comparison with the results for free 
clusters (given in square brackets) shows that the hybridizations with the substrate often lead to a reduction of the Co orbital moments. In the case of monoatomic chains, the effect is particularly strong and has been interpreted as a consequence of the loss of 1D character [172]. In fact, a similar strong reduction of $\left\langle L_{\delta}\right\rangle$ is also observed when one compares monoatomic and biatomic chains. For example, for $N=13$, the Co moment changes from $\left\langle L_{x}\right\rangle=0.42 \mu_{\mathrm{B}}$ in the deposited monoatomic chain (structure (b)) to $\left\langle L_{x}\right\rangle=0.29 \mu_{\mathrm{B}}$ in the diatomic chain which is already not far from our result for the 2D monolayer, namely, $\left\langle L_{x}\right\rangle=0.23 \mu_{\mathrm{B}}$. Similar trends have been observed in experiments on Co chains of various widths grown at the steps of the $\operatorname{Pt}(99$ 7) surface [111].

\subsubsection{Magnetic anisotropy energy}

The interactions between magnetic adatoms and metallic substrates often lead to redistributions of the spin-polarized density and to changes in the electronic structure which affect sensitively the spin-orbit energies. In order to quantify these effects it is interesting to compare the off-plane MAEs $\Delta E_{x z}=$ $E_{x}-E_{z}$ and $\Delta E_{y z}=E_{y}-E_{z}$, and the in-plane MAE $\Delta E_{x y}=E_{x}-E_{y}$ of $\mathrm{Co}_{N}$ clusters deposited on $\operatorname{Pd}\left(\begin{array}{lll}1 & 1 & 1\end{array}\right)$ with the corresponding results for free clusters having the same structure as the deposited ones. The monoatomic free-standing chains show an in-line easy axis with remarkably large MAEs per Co atom $\left[\Delta E_{x z}<0\right.$ with $\left.\left|\Delta E_{x z}\right|=(6.2-20) \mathrm{meV}\right]$. In the case of deposited chains, the interaction with the substrate does not modify the easy axis but reduces the absolute value of the MAEs by an order of magnitude [173]. This is consistent with the reduction of the orbital moments discussed in the previous section.

The 2D clusters and diatomic chains yield $\Delta E_{x z}<0$ in the free standing geometry, which implies that the easy axis is in-plane. The absolute values of the MAEs are much larger than the typical results found in thin films and at surfaces, which are of the order of $0.1-1.0 \mathrm{meV}$. These anisotropy energies are somewhat smaller than in the 1D chains, though mostly of the same order of magnitude $\left[\left|\Delta E_{x z}\right|=0.88-\right.$ $12 \mathrm{meV}]$. As in the 1D case, the largest part of the enhanced MAEs is lost after deposition on Pd $\left[\left|\Delta E_{x z}\right|=0.05-0.70 \mathrm{meV}\right]$. Nevertheless, in the 2D clusters the effects of the interactions with the substrate are qualitatively different since most $2 \mathrm{D} \mathrm{Co}_{N} / \mathrm{Pd}\left(\begin{array}{lll}1 & 1 & 1\end{array}\right)$ present a stable off-plane magnetization direction [151]. This remarkable magnetization reorientation is the result of two main contributions: the changes in the electronic structure of the Co clusters due to cluster-substrate hybridizations, and the anisotropy of the spin-orbit energy at the $\mathrm{Pd}$ atoms of the Co-Pd interface, which carry small induced magnetic moments. Moreover, this effect appears to be a characteristic of the 2D like clusters, since the diatomic chain shows a perpendicular easy axis while the monoatomic chain remains in-line. A detailed analysis of the various local MAEs $\Delta E_{x z}(i)$, presented in Section 5.4.3, indicates that the interface between $\mathrm{Co}$ and $\mathrm{Pd}$ plays a dominant role in the magneto-anisotropic behaviour of the deposited clusters, as already observed in thin Co films on Pd(llll) [170]. Cluster-substrate hybridizations are therefore crucial for the magneto-anisotropic behaviour of magnetic islands deposited on metallic substrates.

Concerning the size dependence of the MAE of $2 \mathrm{D}$ clusters, we would like to point out that the calculated easy axis of the 2D Co monolayer on $\operatorname{Pd}\left(\begin{array}{lll}1 & 1 & 1\end{array}\right)$ is within the surface plane, as observed in experiment [169]. This is in part due to the dipole-dipole energy, that is non-negligible in the case of extended films and that always favours an in-plane magnetization. Moreover, the in-plane direction is further stabilized by the magneto-crystalline anisotropy energy $\Delta E_{x z}$ resulting from SO interactions, which is negative for the 2D monolayer, though rather small. Consequently, a transition from off-plane to in-plane easy axis should be expected to occur in single-layer $\mathrm{Co}_{N}$ as a function of the island size. 
Table 3

Local magnetic properties of an hexagonal $\mathrm{Co}_{7}$ cluster deposited on $\operatorname{Pd}\left(\begin{array}{lll}1 & 1 & 1\end{array}\right)$

\begin{tabular}{llllrr}
\hline$i$ & $2\left\langle S_{i z}\right\rangle$ & $\left\langle L_{i x}\right\rangle$ & $\left\langle L_{i z}\right\rangle$ & $\mu_{i z}$ & $\Delta E_{x z}(i)$ \\
\hline 1 & 1.67 & 0.19 & 0.17 & 1.84 & 0.74 \\
$2-3$ & 1.67 & 0.31 & 0.26 & 1.93 & -0.70 \\
$4-5$ & 1.67 & 0.27 & 0.26 & 1.93 & -0.20 \\
$6-7$ & 1.67 & 0.34 & 0.26 & 0.36 & -0.80 \\
8 & 0.33 & 0.04 & 0.03 & 0.36 & -0.19 \\
$9-10$ & 0.33 & 0.04 & 0.03 & 0.31 & 1.00 \\
11 & 0.27 & 0.02 & 0.04 & 0.31 & 1.28 \\
$12-13$ & 0.27 & 0.04 & 0.04 & 0.22 & 0.48 \\
$14-15$ & 0.19 & 0.03 & 0.03 & 0.22 & -0.03 \\
$16-17$ & 0.19 & 0.03 & 0.03 & 0.22 & 0.39 \\
$18-19$ & 0.19 & 0.03 & 0.03 & 2.40 & 0.28 \\
Average & 2.10 & 0.35 & 0.30 & \\
\hline
\end{tabular}

The spin moments $2\left\langle S_{i z}\right\rangle$, orbital moments $\left\langle L_{i \delta}\right\rangle$ for $\delta=x$ and $z$, and total magnetic moments $\mu_{i z}=2\left\langle S_{i z}\right\rangle+\left\langle L_{i z}\right\rangle$ along the easy axis $z$ are given in Bohr magnetons for the different atomic sites $i$ as labeled in Fig. 33c [151]. The corresponding local magnetocrystalline anisotropy energies $\Delta E_{x z}(i)=E_{x}(i)-E_{z}(i)$ are given in meV (cf. Eqs. (15) and (16))

Moreover, it could be possible that at intermediate sizes the clusters show tilted magnetization with canted easy axes, as already observed in 2D Co-Pd nanostructures [170].

\subsubsection{Local environment effects}

The local formulation given in Section 3 can be exploited to express most relevant magnetic properties as a sum of contributions corresponding to the different atoms $i$ of the cluster and its surroundings. In this way, the observed magnetic behaviour of the deposited clusters can be related to the specific local atomic environments. As an example we consider the hexagonal $\mathrm{Co}_{7}$ cluster on $\operatorname{Pd}\left(\begin{array}{lll}1 & 1 & 1\end{array}\right)$. Table 3 reports the corresponding local spin moments $2\left\langle S_{i z}\right\rangle$, local orbital moments $\left\langle L_{i \delta}\right\rangle$ for $\delta=x, y$, and $z$, and total local moments $\mu_{i z}=2\left\langle S_{i z}\right\rangle+\left\langle L_{i z}\right\rangle$ along the easy axis $\left(\delta_{0}=z\right)$. The labeling of atomic sites is shown in Fig. 33c.

The local spin moments $\left\langle S_{i z}\right\rangle$ at the Co atoms are nearly saturated, and therefore they are quite independent of $i$ and of the particular size or structure of the $\mathrm{Co}_{N}$ cluster. In contrast, the $\left\langle S_{i z}\right\rangle$ induced at the Pd atoms show a very interesting environment dependence. In fact, $\left\langle S_{i z}\right\rangle_{\mathrm{Pd}}$ appears to be approximately determined by the number of Co atoms $z_{i}$ that are $\mathrm{NN}$ of the Pd atom $i$. For $z_{i}=3(i=8-10$ in the hexagon) one obtains $\left\langle S_{i z}\right\rangle=(0.32-0.33) \mu_{\mathrm{B}}$, for $z_{i}=2(i=11-13),\left\langle S_{i z}\right\rangle=0.27 \mu_{\mathrm{B}}$, and for $z_{i}=1$ $(i=14-19),\left\langle S_{i z}\right\rangle=(0.19-0.20) \mu_{\mathrm{B}}$ (see Table 3 and Fig. 33). Similarly strong correlations between local magnetic moments and the immediate chemical environment of the atoms have been already observed in magnetic alloys and thin films which involve, like the present case, non-saturated magnetic moments (e.g. Fe-Al alloys or Fe-Co overlayers) [93,174]. It should be moreover noted that for these small clusters the number of $\mathrm{Pd}$ atoms at the Co-Pd interface is roughly twice the number of Co atoms. Therefore, the induced Pd moments yield a sizeable contribution to the average magnetic moment per Co atom (see Table 2). In this context one should observe that relaxations of the cluster structure or atomic rearrangements at the cluster-substrate interface often modify the magnetic properties, particularly if the relevant local atomic environments change significantly. For instance, in the case of cluster burrowing [175-177] one expects that the total spin polarization of the Pd host should increase, since the number of $\mathrm{Pd}$ atoms in contact with the Co cluster increases. 


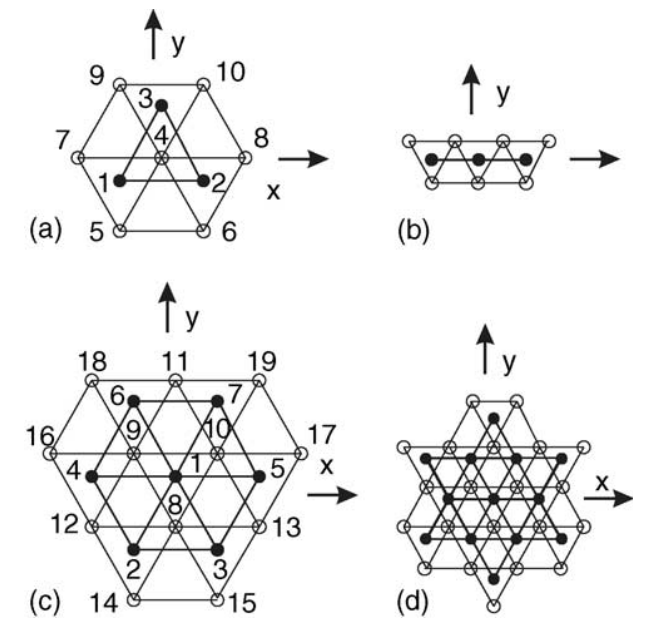

Fig. 33. Illustration of the structures and magnetization directions considered in the calculations on $\mathrm{Co}_{N}$ clusters deposited on $\operatorname{Pd}\left(\begin{array}{lll}1 & 1 & 1\end{array}\right)$. The $z$-direction is perpendicular to the $x y$ surface plane. Open (filled) circles refer to Pd (Co) atoms. The numbers label the different atomic sites $i$ [151].

The local orbital moments $\left\langle L_{i \delta}\right\rangle$ at the Co atoms are typically a factor 2-3 larger than in bulk-Co and in addition show a very interesting environment dependence. One observes that the magnetization direction yielding the largest $\left\langle L_{i \delta}\right\rangle$ is very often different for different atoms $i$. Moreover, the important difference in local coordination number between the central atom $i=1$ and the outer atoms $i=2-7$ results in a different degree of quenching of $\left\langle L_{i \delta}\right\rangle$, the higher coordinated central atom having a smaller orbital moment than the lower coordinated outer ones. For the $\mathrm{Pd}$ atoms at the $\mathrm{Co}-\mathrm{Pd}$ interface the orbital moments are far less important in absolute values $\left[\left\langle L_{i \delta}\right\rangle=(0.01-0.04) \mu_{\mathrm{B}}\right]$, although they still represent about $10-15 \%$ of the total $\mathrm{Pd}$ moments.

The local anisotropy energies $\Delta E_{\delta \gamma}(i)=E_{\delta}(i)-E_{\gamma}(i)$ allow us to analyze the microscopic origin of the perpendicular magnetization in $2 \mathrm{D} \mathrm{Co}_{N}$ on $\mathrm{Pd}\left(\begin{array}{lll}1 & 1 & 1\end{array}\right)$ [151]. The results given in Table 3 show that it is mostly $\mathrm{Pd}$, and not $\mathrm{Co}$, which defines the positive sign of $\Delta E_{x z}$ and thus the perpendicular orientation of the magnetization. For example, in the hexagon, $\Delta E_{x z}(i)<0$ for $i=2-7$ while only $\Delta E_{x z}(1)>0$. The Pd atoms close to $\mathrm{Co}_{N}$ cannot be regarded as a mere source of modifications in the electronic structure of $\mathrm{Co}_{N}$ but rather as important magnetic components on their own. In fact, they carry non-vanishing induced magnetic moments and, as 4 d elements, are subject to significant spin-orbit couplings ( $\left.\xi_{\mathrm{Pd}} \simeq 2 \xi_{\mathrm{Co}}\right)$ [105]. It is therefore reasonable that they contribute to $\Delta E_{x z}$ and to the overall MAE of the system. Note that $\Delta E_{x z}(i)$ of the $\mathrm{Pd}$ atoms is of the same order of magnitude as in the Co atoms, often even larger at Pd (see Table 3). In fact, if one would ignore the Pd contributions to the MAE, the easy axis would be in-plane. The same occurs if the induced magnetic moments or the SO interactions at the Pd atoms are neglected. The central role of the substrate Pd atoms on the MAE is thereby demonstrated. This also confirms the importance of treating the redistributions of the spin-polarized density and the spin-orbit interactions at the same electronic level.

The present discussion should encourage the development of new experimental works as well as further theoretical improvements. For example, it would be worthwhile to investigate more systematically the dependence of the magnetic properties on the geometry of the cluster and its immediate environment. This would be relevant in particular for the comparison with experiment, since the 
morphology of the nanoparticles can be tuned, at least to some extent, by changing the growth and deposition conditions or by subsequent annealing [9,84,109,175-184]. The well-known sensitivity of TM magnetism to the specific local atomic environments should lead to a wide variety of interesting behaviours. Moreover, recent experimental and theoretical studies have revealed remarkable spinreorientation transitions and spin-canted phases at Co-Pd and Co-Pt film interfaces $[169,170,185]$. Therefore, it is reasonable to expect similar phenomena in the case of Co clusters on Pd or Pt surfaces, which deserve detailed investigations both from a fundamental standpoint and in view of applications.

\subsection{Conclusions to supported clusters}

In this section, we have shown experimental results on exposed iron on different surfaces. Small Fe clusters deposited on HOPG with some hundred atoms per cluster show enhanced orbital and spin moments similarly to the results observed for free clusters. When increasing the coverage on the surface, the orbital moment starts to decrease significantly. A coating of these particles with cobalt leads to an increase in the spin moment of the Fe clusters contrary to a capping with copper which slightly reduces the spin moment. This behaviour has been confirmed by calculations. Even large Fe clusters with up to 100,000 atoms per cluster deposited onto a cobalt surface show an enhanced ratio of the orbital to the spin moment when compared to bulk behaviour. For clusters smaller than $9 \mathrm{~nm}$ this value is larger than corresponding data known from surfaces and ultrathin films. Calculations from different groups have clearly demonstrated that enhanced orbital moments can be observed only for the outer two shells of clusters. Thus, this effect has been predicted for small clusters and was confirmed by experimental data from different groups. The very large number of surface atoms even in large clusters leads to enhanced orbital values as displayed in this section. The influence of the bonding to a surface for clusters have been displayed by calculations of cobalt clusters on the $\operatorname{Pd}\left(\begin{array}{lll}1 & 1 & 1\end{array}\right)$ surface. Of course such calculations are restricted to very small clusters. However, the results clearly show the difference between free cobalt clusters, supported clusters and finally the presence of a polarizable surface. The Pd surface causes much higher spin moments in cobalt clusters but generally decreases the orbital moments dramatically when compared to free clusters simply by the presence of atoms. Moreover, the easy magnetization axes in such systems are extremely dependent on the size and the structure of the clusters and its shape on the surface.

\section{Magnetic behaviour of transition metal clusters in matrices}

To understand the magnetic behaviour of dense interacting particle films not only the magnitude of the moments in isolated clusters are required but also other properties such as the magnitude of the magnetic anisotropy and its symmetry. These can be determined by magnetometry measurements in dilute (1-2\% volume fraction) magnetic clusters embedded in non-magnetic matrices prepared by co-depositing the clusters and the matrix.

\subsection{Isolated Fe and Co clusters in Ag matrices}

Fig. 34a shows in-plane magnetic isotherms from an assembly of unfiltered Fe clusters produced by the gas aggregation source (Section 2.1) embedded in $\mathrm{Ag}$ with a volume fraction of $1 \%\left(\mathrm{Fe}_{1} \mathrm{Ag}_{99}\right)$ in the temperature range 50-300 K (symbols) obtained by Binns et al. [122]. They showed that the assembly 

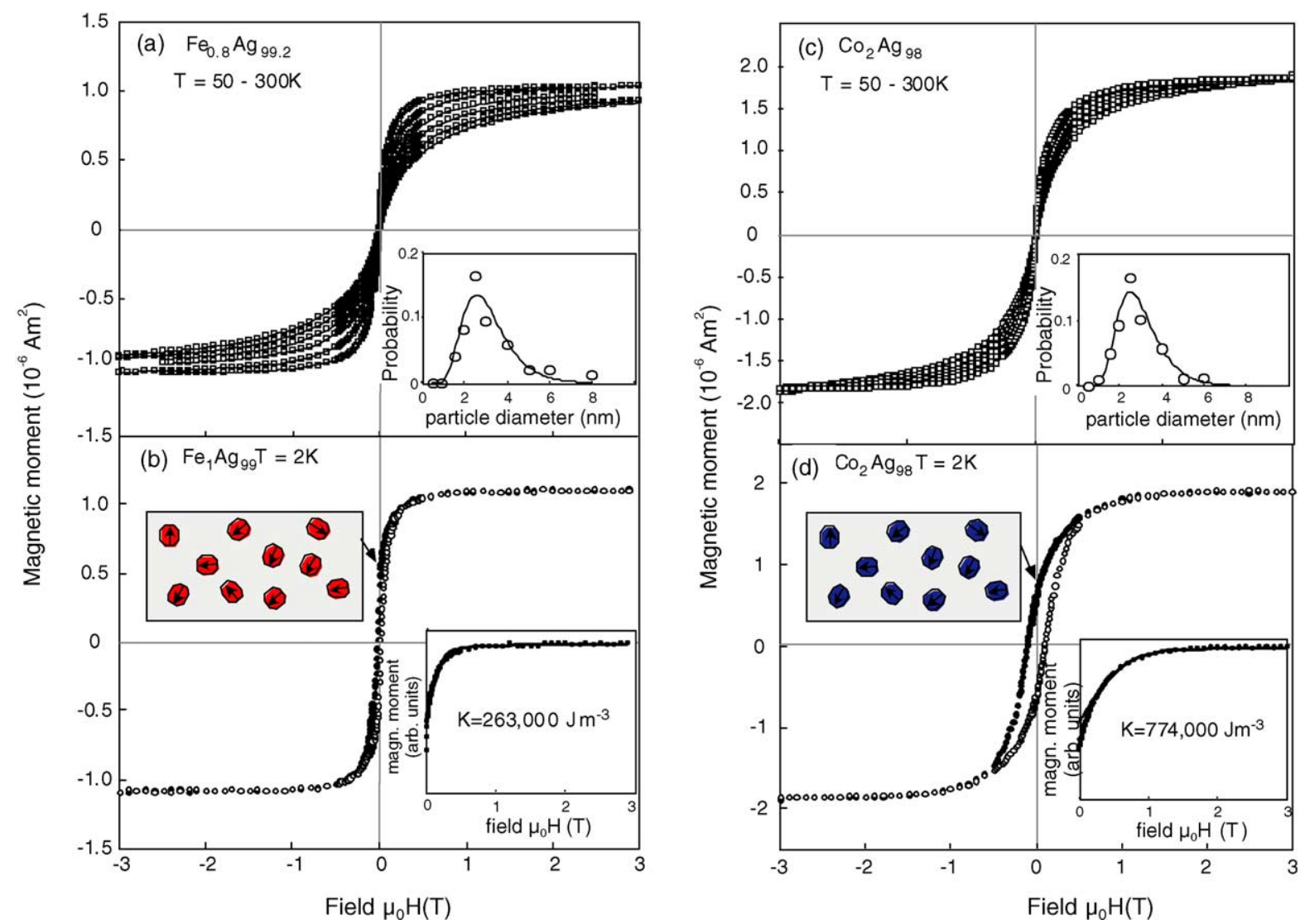

Fig. 34. Magnetization isotherms in the range 50-300 $\mathrm{K}$ of (a) $\mathrm{Fe}_{1} \mathrm{Ag}_{99}$ and (c) $\mathrm{Co}_{2} \mathrm{Ag}_{98}$ cluster-assembled film (open squares) compared to fits by Langevin functions (line) with a size distribution represented by 10 size bins in the range $0.5-8 \mathrm{~nm}$. The insets show the average probability of each bin for the optimum fit to curves at temperatures $T>50 \mathrm{~K}$ (open circles) and the corresponding log-normal distribution (line) with $d_{\max }=2.57 \mathrm{~nm}$ and $\sigma=1.95$ for Fe as well as $d_{\max }=2.45 \mathrm{~nm}$ and $\sigma=1.81$ for Co. (b) In-plane magnetization isotherms at $2 \mathrm{~K}$ of $\mathrm{Fe}_{1} \mathrm{Ag}_{99}$ sample and (d) out-of-plane magnetization isotherms at $2 \mathrm{~K}$ of $\mathrm{Co}_{2} \mathrm{Ag}_{98}$ sample: (filled circles) field sweeping down; (open circles) field sweeping up. The insets show the decay from saturation (filled circles) compared to a calculation (line) assuming a random distribution of uniaxial anisotropy axes (Eq. (19)). The best fit anisotropy constants are displayed in the insets [46].

displays ideal superparamagnetism in the range 50-300 K thus the size distribution of the clusters can be obtained by fitting a set of Langevin functions with different moment values where the amplitude of each Langevin function is a fitting variable. Ten size bins were used in the range $0.5-8 \mathrm{~nm}$ and the average amplitude as a function of particle size (moment) after fitting all data sets is shown in the inset of Fig. 34a. The calculated curves are displayed as lines and the fit is excellent in every case. The size distribution is the usual asymmetric shape and a fitted log-normal distribution (inset) yields a most probable cluster size of $2.57 \mathrm{~nm}$ with a standard deviation of 1.95 giving a median diameter of $3.0 \mathrm{~nm}$. This distribution is similar to those obtained by direct STM imaging of deposited cluster films [122] confirming that the clusters are isolated in the film.

At $2 \mathrm{~K}$, most of the clusters in the $\mathrm{Fe}_{1} \mathrm{Ag}_{99}$ sample are below the blocking temperature and as shown in Fig. 34b the magnetic isotherm develops hysteresis. The remanence, $M_{\mathrm{r}}$, of an assembly of blocked 
particles reveals the symmetry of the anisotropy axes and their distribution in space. For example, uniaxial anisotropy axes give $M_{\mathrm{r}} / M_{\mathrm{S}}=0.5$ if they are randomly distributed over three dimensions and $M_{\mathrm{r}} / M_{\mathrm{s}}=0.71$ if they are distributed over two dimensions in the plane of the applied field. The equivalent values for cubic anisotropy axes are 0.82 distributed over 3D and 0.91 distributed over $2 \mathrm{D}$. The measured remanence is 0.4 and is thus closest to the case for uniaxial anisotropy axes randomly distributed over 3D. The slight discrepancy is due to the smallest clusters in the distribution remaining unblocked at $2 \mathrm{~K}$. For particles with a uniaxial anisotropy randomly oriented in 3D the magnetization between saturation and remanence is obtained at each field value by minimizing over all alignments of the anisotropy axes, the intra-particle energies:

$$
E_{\phi}=K V \sin ^{2}(\phi-\theta)-\mu B \cos \phi
$$

where $K$ is the anisotropy constant, $V$ is the particle volume and $\theta$ and $\phi$ are the angles between the applied field and the anisotropy axis and particle magnetization vector, respectively. The inset in Fig. 34b compares the curve, calculated thus, with the data and it is evident that this simple model reproduces the data accurately. So in zero field the system is a collection of static, randomly aligned cluster giant moments each pointing along the local anisotropy axis. The anisotropy constant is a parameter of the fit and optimizes at $K=2.63 \times 10^{5} \mathrm{~J} / \mathrm{m}^{3}$, which is in reasonable agreement with the value of $K=2.3 \times 10^{5} \mathrm{~J} / \mathrm{m}^{3}$ obtained by a previous SQUID measurement of a similar sample [186], and is about 5 times the bulk value. A previously reported measurement perpendicular to the film plane revealed similar behaviour with a slightly higher anisotropy constant [46], which was attributed to a degree of 'flattening' of the clusters on landing.

An uniaxial anisotropy of the individual clusters is expected as it will be produced by any incomplete atomic shell. Only clusters containing magic numbers of atoms are expected to have a cubic anisotropy but even in this case it is likely to be lost due to other processes during deposition such as collision with the substrate. Magnetic isotherms from a $\mathrm{Co}_{2} \mathrm{Ag}_{98}$ cluster-assembled in the temperature range 50-300 $\mathrm{K}$ are shown in Fig. 34c (symbols) along with the Langevin fits using the procedure described above (lines) [46]. In this case, a departure from ideal superparamagnetism is observed below about $150 \mathrm{~K}$, which was attributed to the higher anisotropy of the Co clusters (see further) requiring a higher temperature to reach the superparamagnetic limit. The size distribution can be obtained by Langevin fits as described earlier but restricted to the data obtained at $T \geq 150 \mathrm{~K}$. The result along with the fitted log-normal distribution is shown in the inset in Fig. 34a and for the Co clusters the most probable size is $2.45 \mathrm{~nm}$ with a standard deviation of 1.81 giving a median diameter of $2.8 \mathrm{~nm}$. The low temperature isotherms from the isolated Co clusters in Ag are shown in Fig. 34d. Again the remanence is slightly less than $50 \%$ indicating that the clusters are randomly oriented over 3D and have a uniaxial anisotropy. The approach to saturation can thus be modeled using Eq. (19) and this is compared to the data in the inset in Fig. 34d. The anisotropy constant that optimizes the fit is two to three times larger than found in the Fe cluster assembly.

\subsection{Magnetic behaviour of interacting cluster assemblies}

All the cluster magnetic properties described in Section 6.1 refer to the behaviour of the isolated particles. In many potential applications of nanoclusters, especially in high-performance materials, the density of clusters in the assembly is sufficiently high to produce significant interactions between the clusters. These can be long range dipolar or short-range exchange forces. We identify two regimes, 
volume fractions below the percolation threshold where there is a mixture of exchange and dipolar interactions and above the percolation threshold where the behaviour of the assembly is dominated by the inter-cluster exchange coupling. There is an abundant literature on interacting magnetic nanoparticle systems in general but few studies have been done on films produced by depositing pre-formed gas-phase nanoclusters. In this review we focus on these.

\subsubsection{Volume fractions below the percolation threshold}

A comprehensive magnetometry study of Fe cluster assemblies in Ag matrices as a function of volume fraction has been reported [122]. The measured magnetic isotherms and FC/ZFC data were analyzed using Monte Carlo simulations. The magnetic state of an individual particle can be described by the Stoner-Wohlfarth model that assumes a coherent rotation of the particle's magnetization. The effective anisotropy for each particle is assumed uniaxial and with an easy axis pointing in a random direction. Exchange coupling occurs between nanoparticles when their surfaces are only a few lattice constants apart so that overlap of the surface electronic wavefunctions is non-negligible. A second type of interparticle interaction is the magnetostatic one, that is always present in nanoparticles. The magnetostatic coupling is treated here within the point dipole approximation that is valid as long as the size of the particles is small compared to their inter-particle distance. Corrections beyond this approximation have been shown to stabilize the ground state properties predicted within the point dipole approximation, thus justifying the treatment of nanoparticles as point dipoles [122,187]. The total energy of an ensemble of interacting nanoparticles has the following form:

$$
E=g \sum_{i, j} \frac{\left(\hat{S}_{i} \cdot \hat{S}_{j}\right)-3\left(\hat{S}_{i} \cdot \hat{R}_{i j}\right)\left(\hat{S}_{j} \cdot \hat{R}_{i j}\right)}{R_{i j}^{3}}-J \sum_{\langle i, j\rangle}\left(\hat{S}_{i} \cdot \hat{S}_{j}\right)-k \sum_{i}\left(\hat{S}_{i} \cdot \hat{e}_{i}\right)^{2}-h \sum_{i}\left(\hat{S}_{i} \cdot \hat{H}\right),
$$

where $\hat{S}_{i}$ is the magnetic moment direction (spin) of particle $i, \hat{e}_{i}$ is the easy axis direction, $R_{i j}$ is the centre-to-centre distance between particles $i$ and $j$, measured in units of the particle diameter $(D)$. Hats in Eq. (20) denote unit vectors. The energy parameters entering Eq. (20) are the Zeeman energy $h=\mu H$, where $\mu=M_{\mathrm{S}} V_{0}$ is the particle magnetic moment, the dipolar energy $g=\mu_{0} \mu^{2} / 4 \pi D^{3}$, the anisotropy energy $k=K_{1} V_{0}$ and the exchange energy $J$. The exchange coupling is non-zero only between particles in contact (nearest-neighbours). Periodic boundary conditions are used and the dipolar interactions are summed to infinite order using Ewald's summation method.

The theory successfully predicts the evolution of all of the magnetic properties with cluster volume fraction up to the percolation threshold, including the observed enhancement of the initial susceptibility of the films at $300 \mathrm{~K}$ and the increased coercivity at $2 \mathrm{~K}$. The evolution of the measured magnetic isotherms with Fe cluster volume fraction at $300 \mathrm{~K}$ is shown in Fig. 35 along with the measured and calculated initial susceptibility (inset).

The basic physical picture provided by the model is that as the volume fraction increases the effects of both dipolar and exchange interactions are evident. The assembly evolves from isolated particles to exchange-coupled agglomerates (super-clusters) where the super-clusters interact with each other via dipolar forces. A simple Curie-Weiss-like extension to the Langevin function appropriate to this regime described by Allia et al. [188] was shown to describe the temperature-dependence of the measured data accurately. This model was used to show that, for example, $3 \mathrm{~nm}$ clusters embedded in Ag matrices at a volume fraction of $10 \%$ behaved like dipolar-coupled particles with a mean diameter of $5.7 \mathrm{~nm}$. Thus, at this density the film consisted of exchange-coupled aggregates with six to seven deposited particles 


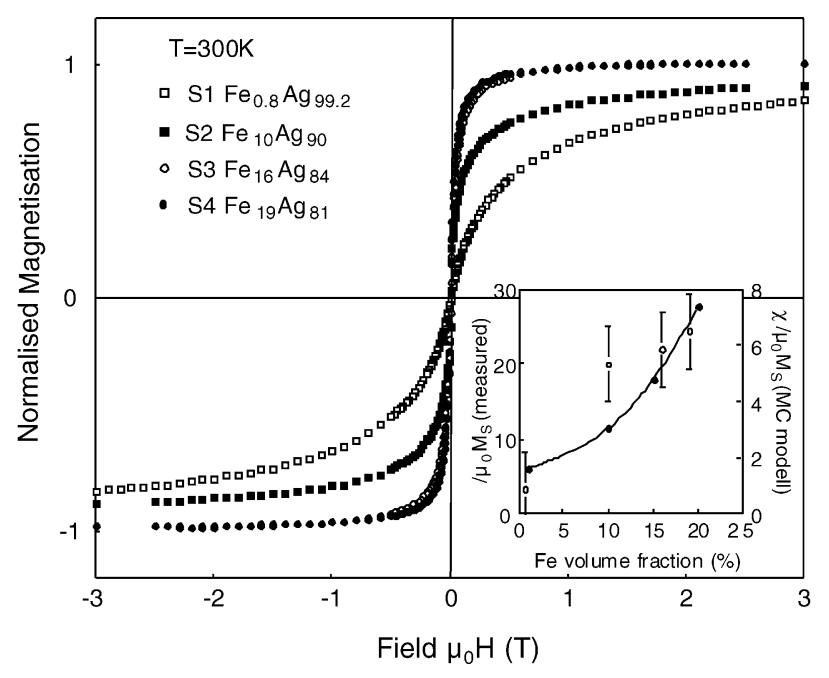

Fig. 35. Magnetization isotherms at $300 \mathrm{~K}$ of films as a function of volume fraction in the intermediate range. The inset compares the measured initial susceptibility with that calculated by the MC simulation as a function of volume fraction (note the different scales) [122].

interacting with each other via dipolar forces. Fig. 36 shows the measured and calculated magnetic isotherms at $2 \mathrm{~K}$ along with the coercivity (inset). A measure of the importance of the exchange coupling between the clusters that one can obtain from the Monte Carlo simulation is the parameter, $b$, given by:

$$
b=\frac{1}{N} \sum_{i}\left|\hat{S}_{i} \cdot \hat{e}_{i}\right|,
$$

where $\hat{S}_{i}$ and $\hat{e}_{i}$ are unit vectors in the direction of the spin and anisotropy axis of the $i$ th cluster and $N$ is the total number of clusters. Eq. (21) thus gives the fraction of projected moments along the local easy axes. A value of 1 indicates complete decoupling of clusters so that all moments are along local easy axes and 0.5 will be found in the case where all neighbouring moments are aligned collinearly by exchange. For the most dilute cluster film, with a cluster volume fraction of $1 \%$, it is found that $b=0.89$ at $H=0$ and $T=5 \mathrm{~K}$ but this drops to 0.63 in a sample with a $10 \%$ volume fraction and drops further towards the exchange-coupled limit as the Fe cluster volume fraction increases. The thin solid lines in Fig. 36 are the MC simulation of the magnetization curves and there is good agreement. Also shown in Fig. 36 are the fits between saturation and remanence using the procedure described in Section 6.1(thick lines) that gives the effective anisotropy constant, which is displayed alongside each sample.

\subsubsection{Volume fractions above the percolation threshold}

Increasing the volume fraction to beyond the percolation threshold produces a correlated super-spin glass (CSSG) state described by Chudnovsky and co-workers [189-193]. Here, the magnetic behaviour at the extreme end of the volume fraction range, that is, pure cluster assemblies with no matrix, will be presented. Two films will be described, that is, a pure Co cluster film of thickness $200 \AA$ and a pure Fe film of thickness $50 \AA$. Both samples were prepared in the UHV sealable ampoule described in Section 4.1 and were transferred into a vibrating sample magnetometer in UHV conditions without a protective 


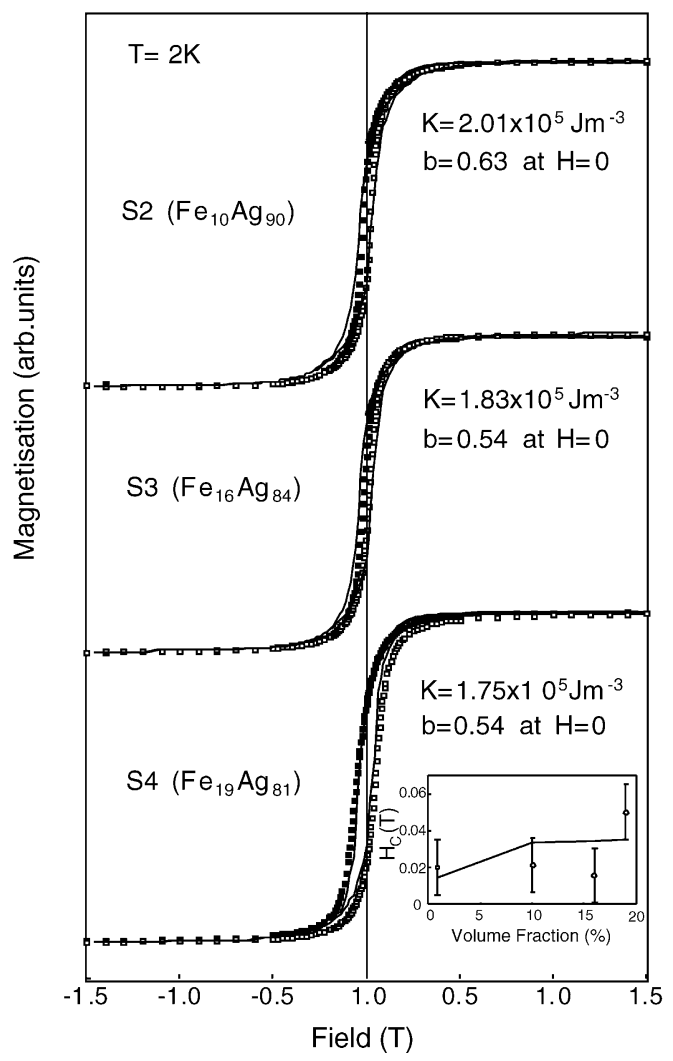

Fig. 36. Isotherms at $2 \mathrm{~K}$ from samples with intermediate volume fractions below the percolation threshold. The symbols are the measured data and the thick lines are fits between saturation and remanence using Eq. (19) from Section 6.1 with the optimal value of the anisotropy constant shown. The thin solid lines are the MC simulations for the full isotherms with the predicted value of $b$ at remanence shown. The inset compares the values of $H_{\mathrm{c}}$ obtained from the measurements and the MC simulation [122].

capping layer. It has been shown that capping layers can significantly alter the magnetic behaviour of cluster films [122]. The behaviour was analyzed using a random anisotropy (RA) model developed by several authors in the last two decades [189-193]. In the RA formalism, the magnetic ground state in a granular film is determined by the relative strength of a random anisotropy field:

$$
H_{\mathrm{r}}=\frac{2 K_{\mathrm{r}}}{M_{\mathrm{S}}}
$$

and an exchange field:

$$
H_{\mathrm{ex}}=\frac{2 A}{M_{\mathrm{s}} R_{\mathrm{a}}^{2}},
$$

where $K_{\mathrm{r}}$ is the (randomly oriented) anisotropy of the grains, $M_{\mathrm{S}}$ is their saturation magnetization, $A$ is the exchange constant for the interaction between the grains and $R_{\mathrm{a}}$ is the nanometer sized region over which the local anisotropy axis is correlated, i.e. the characteristic grain size. In this case, the grain size is known and can be taken as the median size in the log-normal distributions shown in the insets in Fig. 34, that is, 


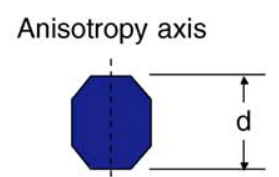

(a)

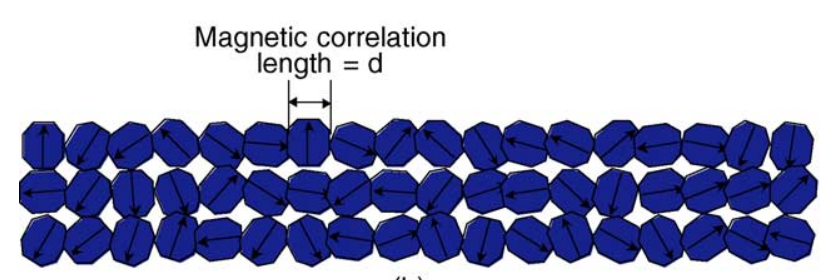

(b)

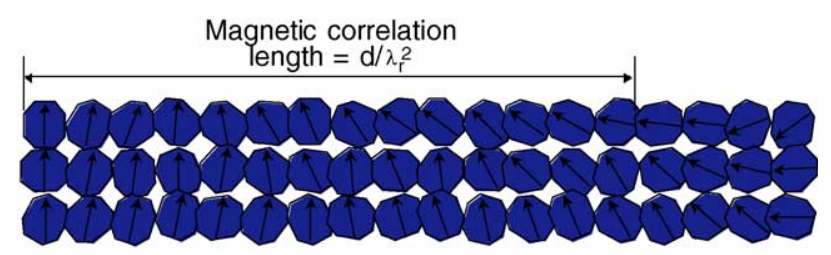

(c)

Fig. 37. (a) Schematic representation of a magnetic nanoparticle with a uniaxial anisotropy axis (represented by a slight elongation). (b and c) A stack of particles with randomly oriented anisotropy axes. In (b), $\lambda_{\mathrm{r}}>1$ and the magnetization vector points along the local anisotropy axis so the magnetic correlation length is a single particle diameter. In $(\mathrm{c}), \lambda_{\mathrm{r}}<1$ and the magnetic vectors are nearly aligned. The random perturbation from perfect alignment results in a finite magnetic correlation length that is a factor $1 / \lambda_{\mathrm{r}}^{2}$ larger than a single particle [46].

3.0 and $2.8 \mathrm{~nm}$ for $\mathrm{Fe}$ and Co clusters, respectively. Their relative strength of the exchange and anisotropy fields is given by the dimensionless parameter:

$$
\lambda_{\mathrm{r}}=\frac{H_{\mathrm{r}}}{H_{\mathrm{ex}}}=\frac{K_{\mathrm{r}} R_{\mathrm{a}}^{2}}{A} .
$$

The model was developed to describe amorphous films in which a local, randomly-oriented, anisotropy is due to local atomic order. It is even better suited to providing a description of the magnetization in clusterassembled films in which the distance $R_{\mathrm{a}}$ over which an anisotropy axis is correlated is well defined (i.e. the particle diameter). In addition, as pointed out by Löffler et al. [194], the exchange interaction at the boundaries between particles is weaker than the intra-particle atomic exchange further reinforcing the image of separate but interacting particles.

For $\lambda_{\mathrm{r}}>1$ the magnetic correlation length at zero field is $R_{\mathrm{a}}$, and the magnetic vector in each particle points along the local intra-particle anisotropy axis. Note that in an arrow representation this state would be identical to that in isolated non-interacting particles at absolute zero. This regime is illustrated in Fig. 37b. With increasing inter-particle exchange (or decreasing intra-particle anisotropy) the configuration becomes a correlated super-spin glass in which the magnetization vector in neighbouring particles is nearly aligned but the random deviation of the moments from perfect alignment produces a smooth rotation of the magnetization throughout the system with a magnetic correlation length that is a factor $1 / \lambda_{\mathrm{r}}^{2}$ larger than the particle diameter. This regime is illustrated in Fig. 37c. The disordered CSSG state is fragile and application of a small field produces a 'ferromagnet with wandering axes' (FWA) 


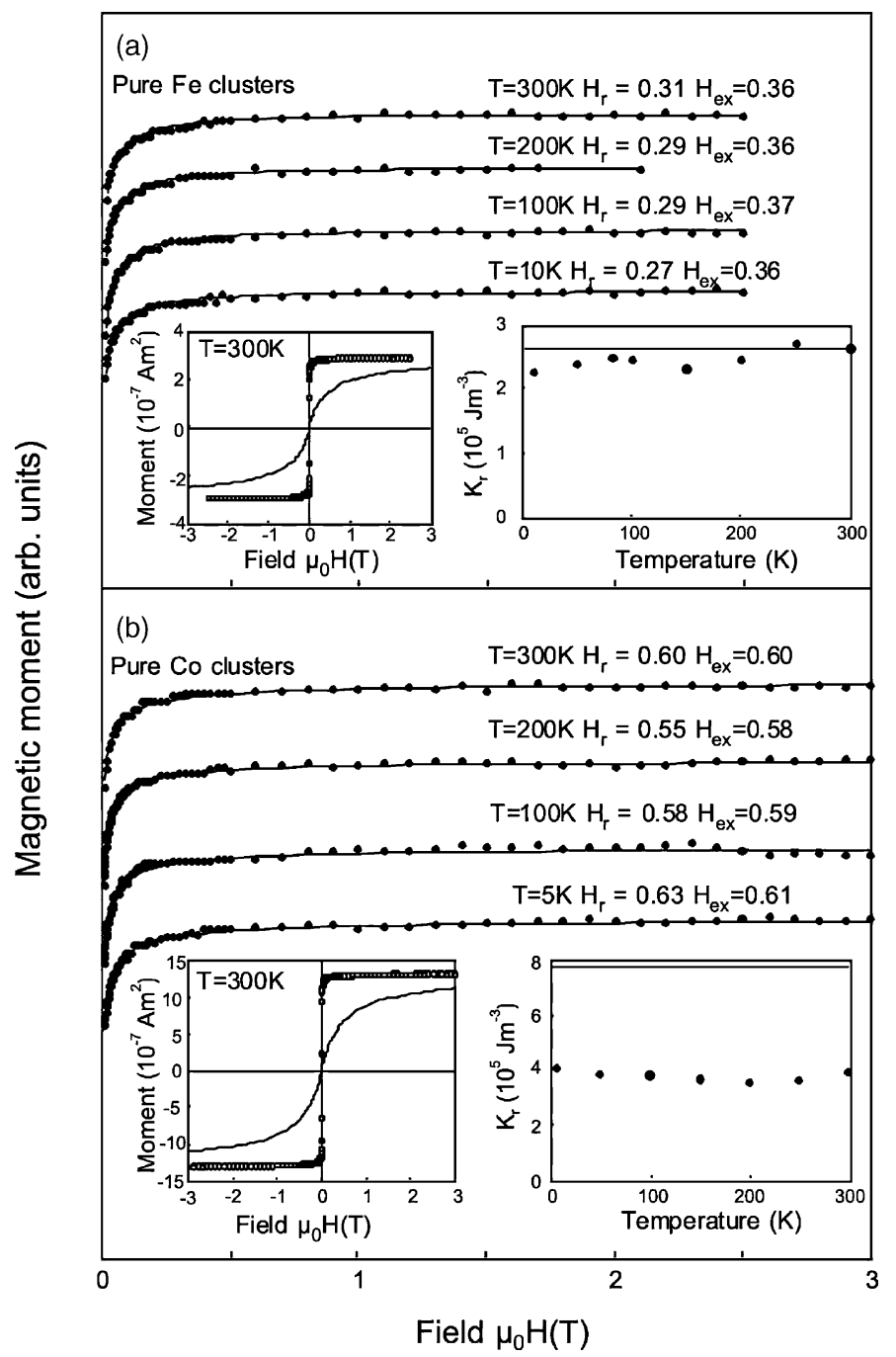

Fig. 38. (a) Approach to saturation of $5 \mathrm{~nm}$ thick pure Fe cluster film for temperatures in the range 10-300 K (filled circles) compared to a calculation using the RA model with the parameters shown (line). The left inset shows the full magnetization curve (circles) of the dense cluster film compared to that of isolated clusters at $300 \mathrm{~K}$. The right inset shows the variation of the random anisotropy (evaluated from $H_{\mathrm{r}}$ and Eq. (22)) with temperature (filled circles). The grey line shows the value for the isolated clusters. (b) As (a), but for the $20 \mathrm{~nm}$ thick pure Co cluster film sample [46].

[190]. The randomized nanoscale anisotropy results in a very high susceptibility of cluster-assembled films as illustrated in the left hand insets in Fig. 38a and b. These compare the magnetization curves of pure cluster-assembled Fe and Co films (dots) at $300 \mathrm{~K}$ with the magnetization of isolated Fe and Co clusters embedded in Ag (lines). According to Chudnovsky [193] the approach to saturation of a system, whose ground state is a CSSG, is given by:

$$
M=M_{\mathrm{s}}\left(1-\frac{1}{32} \frac{\lambda_{\mathrm{r}}^{2}}{h_{\mathrm{ex}}} \int_{0}^{\infty} \mathrm{d} x C(x) x^{2} K_{1}\left[x h_{\mathrm{ex}}\right]\right)
$$


in two dimensions and

$$
M=M_{\mathrm{s}}\left(1-\frac{1}{30} \frac{\lambda_{\mathrm{r}}^{2}}{h_{\mathrm{ex}}} \int_{0}^{\infty} \mathrm{d} x C(x) x^{2} \exp \left[x h_{\mathrm{ex}}\right]\right)
$$

in three dimensions. In Eqs. (25) and (26), $h_{\mathrm{ex}}=H / H_{\mathrm{ex}}, K_{1}$ is the modified Hankel function and $C(x)$ is the correlation function for the anisotropy axes with $x$ in units of $R_{\mathrm{a}}$. In an amorphous metal, $C(x)$ will be a smoothly decreasing function with a characteristic decay distance of $R_{\mathrm{a}}$ while in a cluster-assembled film consisting of mono-sized particles it will be a step function cutting off at $x=1$. The $2 \mathrm{D}$ form (Eq. (25)) for the approach to saturation was used for the Fe cluster sample as it was only two cluster layers thick whereas the 3D form (Eq. (26)) was used for the much thicker Co cluster film.

With suitable constraints on the parameters $H_{\mathrm{ex}}, H_{\mathrm{r}}$ obtained from analyzing the isolated clusters the data was fitted in the range between $0.85 M_{\mathrm{S}}$ and $M_{\mathrm{S}}$, using Eqs. (25) and (26) and the results for temperatures in the range 5-300 K are shown in Fig. 38a and b. The first observation is the excellent agreement between the data and the RA model at all temperatures for both films reinforcing the assertion that the cluster layers form a CSSG in their ground state. It is found that in both cases the values of $H_{\mathrm{ex}}$ and $H_{\mathrm{r}}$ change little with temperature, which is just confirmation of the observation that the shape of the curves appears to be independent of temperature. The value of the random anisotropy, $K_{\mathrm{r}}$, obtained from $H_{\mathrm{r}}$ and Eq. (22) is shown in the right insets and compared to the values for the isolated clusters. For the Fe cluster film, the random anisotropy is close to the isolated cluster value whereas for the Co cluster film it is significantly smaller. The exchange fields are $\approx 0.36$ and $\approx 0.6 \mathrm{~T}$ for the Fe and Co cluster films, respectively, corresponding to an exchange constant (from Eq. (24)) of $A \approx 3 \times 10^{-12} \mathrm{~J} / \mathrm{m}$ for both films, which is much less than the bulk value as expected.

\section{Structural and magnetic properties of binary alloy clusters}

Pure magnetic transition metal nanostructures studied extensively up to now have significantly contributed to a better understanding on the relation between structure and magnetic properties for isolated individual particles taking into account surfaces and interface interaction effects. The use of binary alloy clusters opens new fields to tailor magnetic properties. Using binary $3 \mathrm{~d}$ transition metal alloys with a very high magnetic moment it might be possible to enhance the magnetic moments in nanoparticles above values known from pure materials. FeCo alloys are usually soft-magnetic materials with high saturation magnetizations and small coercive forces. These materials allow a reversal of the magnetization direction already at low magnetic fields. Such nanoparticles, combined with an enhancement of the magnetic moments above bulk material values, would be very interesting for technical applications, e.g. in read-write heads of magnetic storage devices. Section 7.1 gives an introduction to the magnetic properties of FeCo nanoparticles.

Unfortunately, the superparamagnetic limit leading to very low magnetic blocking temperatures prevents the use of pure or binary soft-magnetic transition metal particles with sizes lower than about $10 \mathrm{~nm}$ for applications in high density storage media [195]. A challenge in this field of magnetic nanostructures applications is to synthesize stable nanosize magnetic objects with blocking temperatures around or above room temperature. First interesting investigations with respect to technical applications have been carried out on FePt alloy clusters. Sun et al. [11] showed that monodispersed FePt clusters can be deposited in regular arrays on surfaces by wet-chemical processes. However, any technical application 
regarding magnetic data storage requires FePt clusters to be ordered in the $\mathrm{L} 1_{0}$ structural phase which can be achieved by thermal treatment. This challenge has been addressed successfully by Weller and coworkers using a cluster gun in combination with in situ heating prior to deposition [40]. Very recent calculations from Burkert et al. [196] showed that also FeCo films, which are usually soft-magnetic, exhibit very high magnetic anisotropy energies (even higher than FePt) in a tetragonal distortion phase and might be useful candidates for many applications. However, in the following, we will focus on mixed clusters of intermetallic compounds with strong magneto-crystalline anisotropies such as rare earthtransition metal alloys that have been produced with a laser vaporization source. Examples for this research project, which is still at the beginning, are CoSm clusters (cf. Section 7.2) as well as CoAg and CoPt clusters (see Section 7.3). It should be noted that the magnetic properties of such mixed clusters strongly depend on their chemical composition and geometric structure. The last subsection (cf. Section 7.4) presents calculations on the orbital and spin moments of CoRh clusters in various compositions and stoichiometries.

\subsection{Binary FeCo alloy clusters}

This section is related to soft-magnetic 3d-binary clusters with very high magnetization values. Today, possible applications of such bulk materials are solenoids where an extremely high saturation magnetization and a small coercive force is required. One of the materials, which are commercially used for this purpose, consists of nearly equal parts of iron and cobalt. For the experiments, a piece from a commercial product (VACOFLUX 50, distributed by the Vacuumschmelze GmbH, Germany [197]) was used as a target for producing nanoparticles using the ACIS source (see Section 2.2). In detail, the target consists of a $\mathrm{Fe}_{50} \mathrm{~V}_{2} \mathrm{Co}_{48}$ alloy. The structural properties of FeCo alloy nanoparticles from this target have been investigated by TEM, HRTEM incl. EDX and AFM, the magnetic behaviour using XMCD at the storage ring BESSY in Berlin. Fig. 39 displays in the left part a size distribution of FeCo alloy clusters (mean size: $9.5 \mathrm{~nm}$ ) obtained from conventionally TEM measurements. Additionally, high-resolution TEM and EDX measurements have been carried out in the University of Duisburg-Essen (Germany) (cf. HRTEM pictures in the right part of Fig. 39). A chemical composition of $\mathrm{Fe}_{56} \mathrm{Co}_{44}$ has been obtained from EDX.
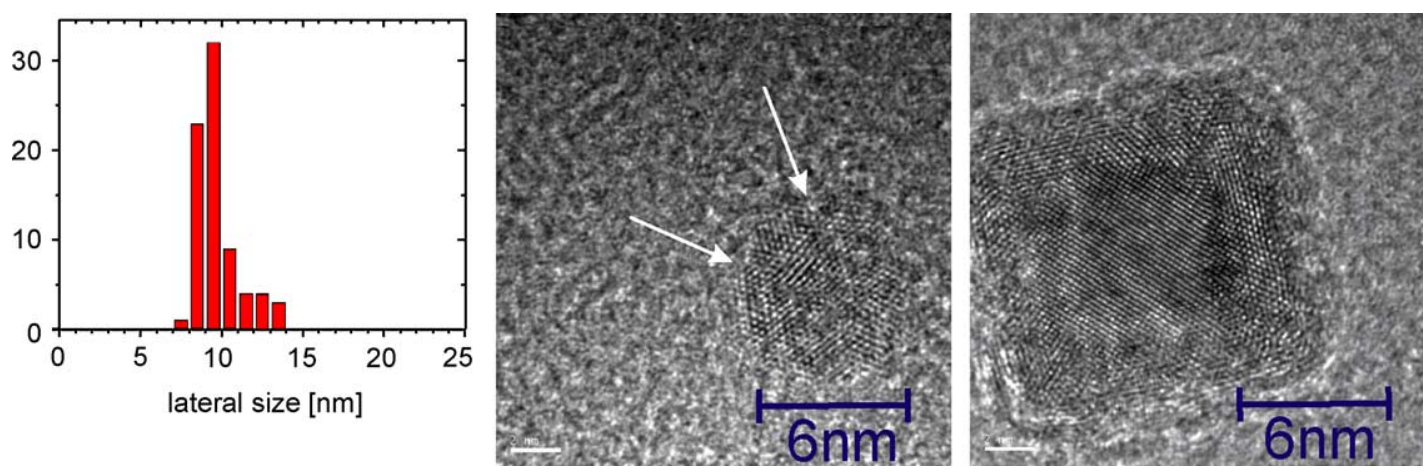

Fig. 39. Left part: size distribution of mass-filtered FeCo clusters with a mean size of $9.5 \mathrm{~nm}$. Right part: high-resolution TEM images taken from two different mass-filtered FeCo clusters with mean sizes of $6 \mathrm{~nm}$ (middle) and $9.5 \mathrm{~nm}$ (right). The HRTEM measurements have been carried out by Mrs. Stappert at the physics department of the University of Duisburg-Essen, Germany. Arrows indicate grain boundaries $[49,198]$. 


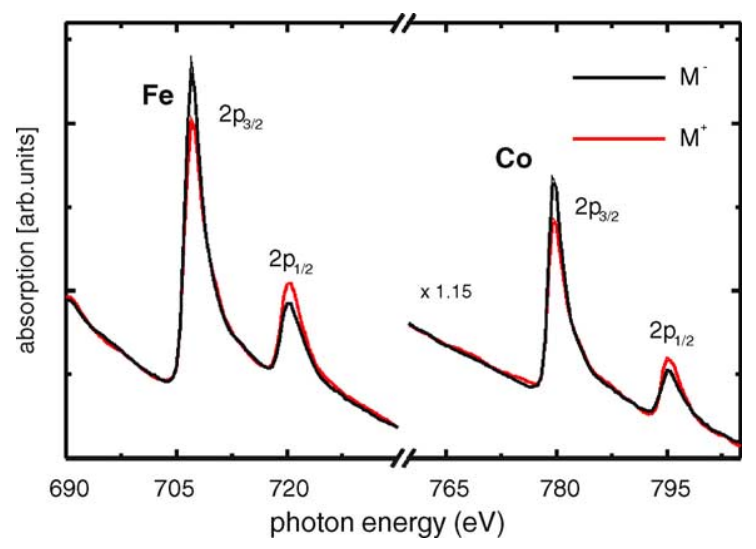

Fig. 40. Photoabsorption spectra for opposite magnetization directions $\left(\mathrm{M}^{+}\right.$and $\left.\mathrm{M}^{-}\right)$taken in remanence with circularly polarized radiation from the $2 p$ core levels of FeCo particles deposited on a silicon wafer [198].

For analyzing the magnetic properties the technique of X-ray magnetic circular dichroism in photoabsorption has been applied which offers information on the orbital and magnetic moments. For a detailed analysis, mass-filtered FeCo clusters with a mean size between 7.5 and $12 \mathrm{~nm}$ have been deposited onto silicon wafers and onto ferromagnetic $\mathrm{Ni}\left(\begin{array}{lll}1 & 1 & 1\end{array}\right)$ on $\mathrm{W}\left(\begin{array}{lll}1 & 1 & 0\end{array}\right)$ films under UHV conditions. The data have been recorded in remanence after magnetization with an external magnet. Photoabsorption spectra from $12 \mathrm{~nm}$ FeCo clusters deposited onto a silicon wafer are displayed in Fig. 40 in the energy range of the respective $2 \mathrm{p}$ absorption levels, i.e. between 690 and $810 \mathrm{eV}$. The experimental data clearly show the absorption edges of both materials (Fe and Co) with nearly identical intensities, $\mathrm{M}^{+}$ and $\mathrm{M}^{-}$denote data taken for opposite magnetization directions in remanence. The intensity differences reveal a ferromagnetic coupling of $\mathrm{Fe}$ and $\mathrm{Co}$ in the nanoparticles. However, the spectra do not reflect a saturated magnetization states, element-specific hysteresis curves taken at the Fe and Co $2 \mathrm{p}_{3 / 2}$ levels display a remanence $M_{\mathrm{r}}$ of about $20 \%$ with respect to the saturation magnetization $M_{\mathrm{s}}$. The corresponding hysteresis curves for iron and cobalt are shown in Fig. 41. Both hysteresis curves show a nearly identical slope with a saturation field of about $H_{\mathrm{s}}=40 \mathrm{mT}$. These results hint at a parallel alignment of the spins in both materials ( $\mathrm{Fe}$ and $\mathrm{Co}$ ), i.e. a strong ferromagnetic coupling as one would expect from the enhanced total magnetic moment in FeCo alloys.

In order to investigate the orbital and spin moments it is necessary to saturate the nanoparticles remanently, either in an externally applied magnetic field or by a strong ferromagnetic coupling to a magnetic substrate. For the results presented here the FeCo alloy clusters have been deposited onto a thin $\mathrm{Ni}\left(\begin{array}{lll}1 & 1 & 1\end{array}\right)$ film evaporated on W(1 110$)$ similar to Section 5.2. Details on the epitaxial growth and magnetic properties of nickel films on W(1 110$)$ can be found elsewhere [199-201], however, these films can be magnetized remanently in-plane along the W[ $\left.\begin{array}{lll}0 & 0 & 1\end{array}\right]$ axis. Fig. 42 shows the respective photoabsorption spectra of the three metals involved, Fe, Co and $\mathrm{Ni}$. The experimental results obtained from Fig. 42 indicate high magnetic moments. By applying the sum rules, one can calculate element-specific the spin and orbital moments. The Slater-Pauling curve predicts an averaged total magnetic moment of about $2.3 \mu_{\mathrm{B}}$ for equal contributions of iron and cobalt in the binary alloy, the maximum value is reached for an alloy with a larger contribution of $\mathrm{Fe}$ (about 70\%). These calculations are in good agreement with data from the company that distributes the bulk alloy material giving a value of $2.3 \mathrm{~T}$ for the magnetic polarization (the magnetic polarization in $\mathrm{T}$ is nearly identical to the total magnetic moment in $\mu_{\mathrm{B}}$ ). 


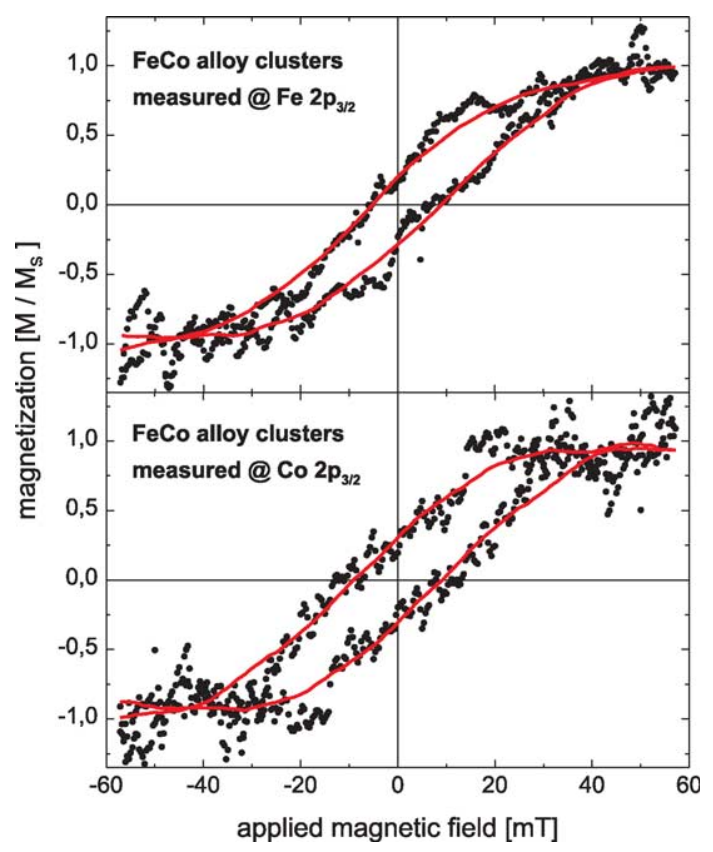

Fig. 41. Element-specific hysteresis curves recorded at the Fe and Co 2 $\mathrm{p}_{3 / 2}$ core levels of FeCo alloy nanoparticles. Upper part: hysteresis curve sensitive to iron, lower part: curve sensitive to cobalt [198].

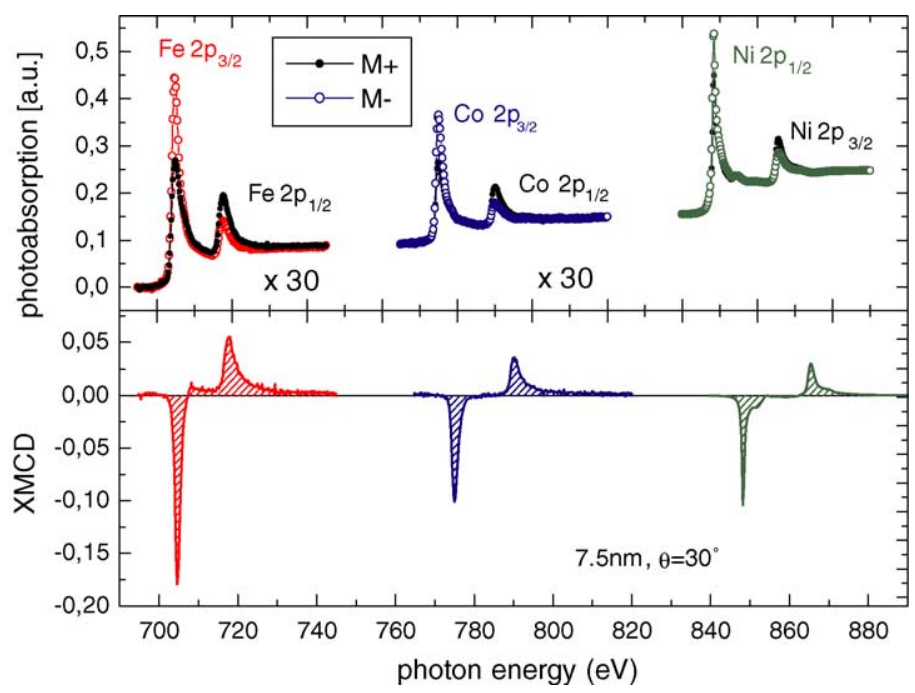

Fig. 42. Upper part: photoabsorption spectra of $7.5 \mathrm{~nm}$ FeCo particles deposited on a Ni(l 111$)$ film on W(1 10$)$ taken in remanence with circularly polarized radiation from the $2 \mathrm{p}$ core levels (for opposite magnetization directions $\mathrm{M}^{+}$and $\mathrm{M}^{-}$) (cf. also [37]). The iron and cobalt spectra have been enlarged by a factor of 30. Lower part: corresponding XMCD data (intensity differences) [37]. 


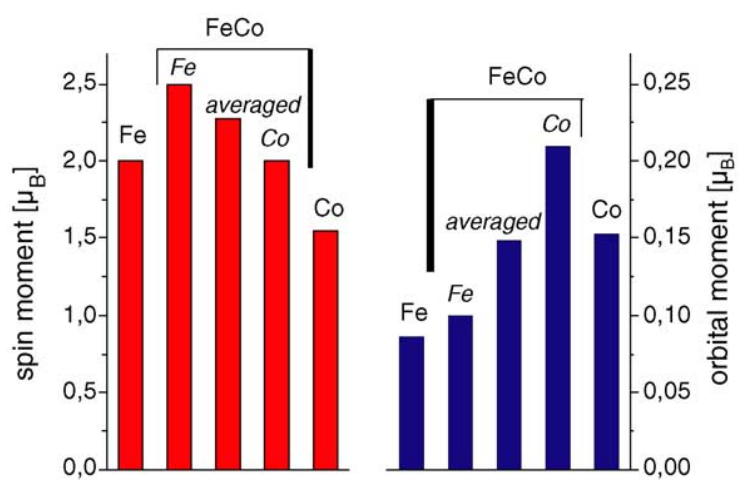

Fig. 43. Element-specific spin (left part) and orbital moments (right part) of mass-filtered FeCo alloy clusters (size: $7.5 \mathrm{~nm}$ ) on $\mathrm{Ni}\left(\begin{array}{lll}1 & 1 & 1\end{array}\right) / \mathrm{W}\left(\begin{array}{lll}1 & 1 & 0\end{array}\right)$ in comparison to pure Fe and Co bulk values (left and right columns in each graph). The bars in the center show the averaged value from the iron and cobalt contribution.

A first analysis of our experimental results indeed confirms enhanced spin and orbital moments in both constituents (Fe and $\mathrm{Co}$ ) (see Fig. 43), when compared to their bulk values (Fe: left side; Co: right side in each graph). The data show a spin moment in the Fe contribution of about $2.5 \mu_{\mathrm{B}}$, the corresponding bulk value is close to $m_{\mathrm{S}}=2.0 \mu_{\mathrm{B}}$. The same holds for the cobalt spin moment which reaches nearly $m_{\mathrm{S}}=$ $2.0 \mu_{\mathrm{B}}$ in the $\mathrm{Fe}_{56} \mathrm{Co}_{44}$ alloy clusters on $\mathrm{Ni}$ film. The bars in the middle of Fig. 43 show the averaged values of the weighted $\mathrm{Fe}$ and Co contribution to the alloy clusters. The total spin moments are in good agreement with theoretical values given in [202]. According to our knowledge no chemically resolved experimental data exist for the spin and orbital magnetic moments of bulk FeCo alloys. In order to distinguish between enhancements originating from the FeCo alloy material itself and from the effect related to clusters we compare our results to calculations from Ebert and Battocletti [202] on a $\mathrm{Fe}_{50} \mathrm{Co}_{50}$ bulk alloy. We find a good agreement for the Fe spin and orbital moment, while the experimental value for the Co moments exceed the calculated values by about $15 \%$ (spin moment) and $50 \%$ for the orbital moment. Taking into account our experimental uncertainty and the typical underestimation of the orbital moment known from theory, we actually cannot claim to have observed enhanced magnetic moments in our FeCo alloy particles. However, the experiments on such alloy clusters have shown that at least the corresponding bulk values are reached. Further studies on systems with different stoichiometries will show if FeCo clusters and particles are of technological relevance.

\subsection{Structural and magnetic properties of CoSm clusters}

This section deals with binary alloy clusters formed from a $3 \mathrm{~d}$ transition metal (here: cobalt) in combination with a rare-earth metal (in this case, $\mathrm{Sm}$ ). In particular, $\mathrm{SmCo}_{5}$ nanoclusters (mean diameter $\approx 3-4 \mathrm{~nm}$ ) prepared from a bulk $\mathrm{SmCo}_{5}$-target mounted in the laser vaporization source (see Section 2.3) have been deposited or embedded in the low-energy regime on various substrates/matrices for structural and magnetic studies [59]. As mentioned in Section 2.3, mixed clusters prepared by this technique exhibit the same composition as the target, however a poor crystallinity as revealed by TEM measurements and a segregation of samarium atoms at the cluster surface is the cause of a loss of magnetization in the as deposited cluster films compared to the bulk $\mathrm{SmCo}_{5}$-phase. Post annealing treatments in UHV up to $840 \mathrm{~K}$ on films of isolated clusters embedded in niobium (cluster concentration $=5 \mathrm{vol} \%$ ) seem to improve the 


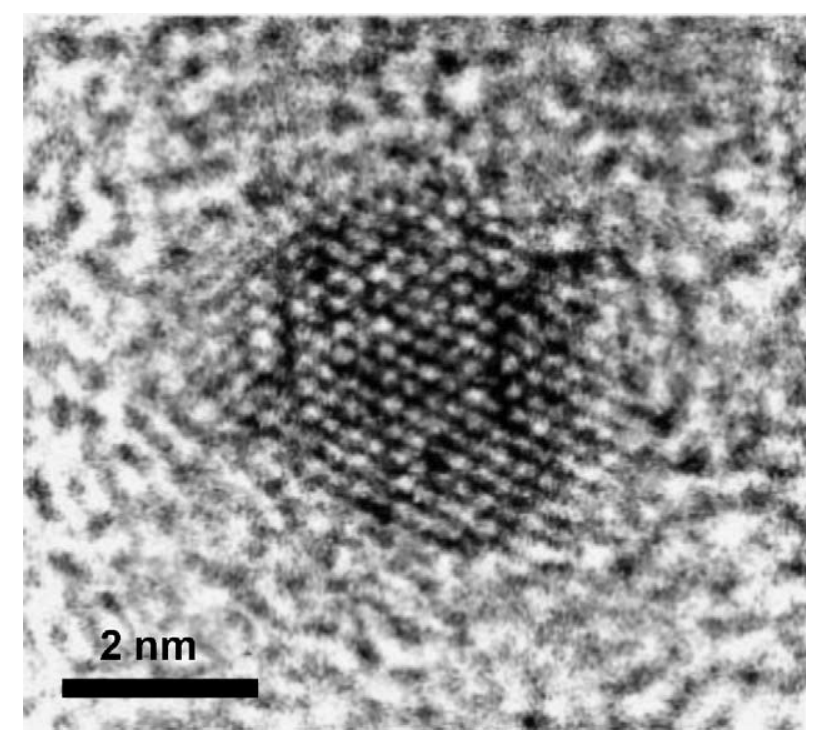

Fig. 44. HRTEM image of a Co-Sm mixed cluster deposited on an amorphous carbon coated grid at room temperature and subsequently annealed at $840 \mathrm{~K}$ in UHV. However, it was difficult to determine the nature of the crystalline phase of annealed clusters since the symmetry and lattice parameters differ from the well-known bulk phases [59].

crystallinity of the Sm-Co nanoparticles, cf. Figs. 44 and 45. A detailed TEM analysis of post annealing treatment has been carried out on isolated CoSm nanoparticles on TEM grids, ordered TEM images of CoSm nanoparticles could mainly be observed after annealing (see Fig. 44). As a consequence of the post annealing process a significant increase of the magnetic anisotropy is observed with the origin of a blocking temperature $T_{\mathrm{B}}$ being larger than $300 \mathrm{~K}$. This is illustrated in Fig. 45 showing the characteristic evolution of the magnetization loops with a significant increase of the coercive field upon annealing for a Sm-Co clusters/Nb matrix film. Note that the choice of a niobium matrix for the annealing experiments is directed by the non-miscible character of this element with samarium, which can be favourable during annealing treatments to avoid the formation of an alloy shell. Since in the as deposited samples samarium atoms are segregated at the cluster/matrix interface one can imagine that during thermal treatments they prefer to
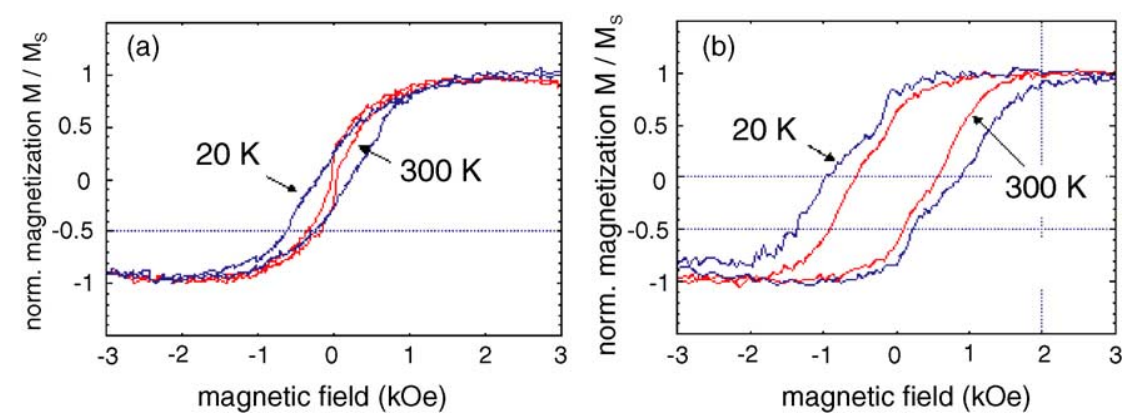

Fig. 45. SQUID magnetization loops measured on (a) an as deposited film of Co-Sm clusters embedded in niobium (concentration $=5$ vol.\%), and (b) the same film subsequently annealed in UHV at $840 \mathrm{~K}$ for $30 \mathrm{~min}$. 
redistribute inside the clusters rather than to diffuse in the surrounding $\mathrm{Nb}$ matrix. In the same time, the mean size of the embedded Sm-Co clusters slightly increases which cannot explain the $T_{\mathrm{B}}$ increase up to room temperature or more. Finally, only the efficient role of samarium atoms in the Co lattice of the clusters can be considered in this case to explain such large magnetic anisotropy/blocking temperature increase. The final association of functionalized clusters (i.e. high magnetic anisotropy cluster blocked at room temperature such as mixed Co-Sm clusters) on functionalized substrate (i.e. 2D-ordered cluster arrays, see Section 2.3) will be the ultimate stage prior to the realization of novel nanomagnetic devices with very high integration density in the Tbits/in. ${ }^{2}$ range.

\subsection{Structural and magnetic properties of mixed Co-Ag and Co-Pt clusters}

Taking advantage of segregation effects expected in some bimetallic nanosize systems, Co-Ag and Co-Pt mixed clusters have been investigated more recently to produce core-shell clusters with strong surface/interface effects on the magnetic anisotropy. In the case of mixed Co-Ag clusters the segregation towards the surface of silver atoms is expected [57]. Indeed, from a thermodynamic point of view, the lower surface energy of silver compared to cobalt $\left(\gamma(\mathrm{Ag})=1250 \mathrm{~mJ} / \mathrm{m}^{2}\right.$ and $\left.\gamma(\mathrm{Co})=2550 \mathrm{~mJ} / \mathrm{m}^{2}\right)$, the higher Ag atomic radius ( $r_{\mathrm{Ag}}=1.13 \AA$ and $r_{\mathrm{Co}}=0.74 \AA$ ), and the non-miscible character of the $\mathrm{Co}-\mathrm{Ag}$ pair should favour the silver surface-segregation. Nevertheless, for a given $\mathrm{Co}_{50} \mathrm{Ag}_{50}$ bulk-target mounted in the laser vaporization source, we observe from RBS measurements that the mean composition of the $\mathrm{Co}-\mathrm{Ag}$ cluster films is strongly related to the source operating conditions (see Table 4). Finally, a nanosecond pulsed laser to vaporize the Co-Ag target in the cluster source associated to a continuous gas flow injection seem necessary to obtain clusters films with the same composition than the target. The following reported results concern only this type of stoichiometric clusters. From TEM observations, the size distributions of supported clusters also seem to be dependent on the source operating conditions yielding a mean size in the range $2-4 \mathrm{~nm}$ in all cases (Fig. 46). The calculated number of atoms located at the cluster surface are given in Table 5 assuming perfect fcc-truncated octahedrons with different sizes in the range 1.6-4 $\mathrm{nm}$. If we assume a complete $\mathrm{Ag}$ atom segregation at the cluster surface to form perfect core-shell cluster types one can underline that the Ag concentration is a critical parameter in the case of the smallest clusters for $\mathrm{Co}_{50} \mathrm{Ag}_{50}$ compound systems. However, the core-shell structure for small Co-Ag clusters is probably not the only one to be considered. Molecular dynamic simulations are in progress to predict the most probable structure in this case.

Magnetometry measurements on individual CoAg clusters have been performed using the highly sensitive microSQUID technique described in details elsewhere [204]. For that purpose, CoAg clusters

Table 4

Mean composition of the deposited cluster- assembled films measured by RBS as a function of the operating conditions of the laser vaporization cluster-source in which a $\mathrm{Co}_{50} \mathrm{Ag}_{50}$ bulk compound target is mounted

\begin{tabular}{llr}
\hline Source operating conditions & $\begin{array}{l}\text { Mean composition of the } \\
\text { deposited cluster films (\%) }\end{array}$ \\
\cline { 2 - 3 } & Co & $\mathrm{Ag}$ \\
\hline $3 \mu$ s-pulsed Ti:Sa laser at $10 \mathrm{~Hz}$ and pulsed pure He gas injected & 90 & 10 \\
$3 \mu$ s-pulsed Ti:Sa laser at $10 \mathrm{~Hz}$ and continuous pure He gas flow & 74 & 26 \\
80 ns-pulsed Ti:Sa laser at $10 \mathrm{~Hz}$ and continuous pure He gas flow & 90 & 10 \\
80 ns-pulsed Nd:YAG Laser at $10 \mathrm{~Hz}$ and continuous pure He gas flow & 74 & 26 \\
\hline
\end{tabular}




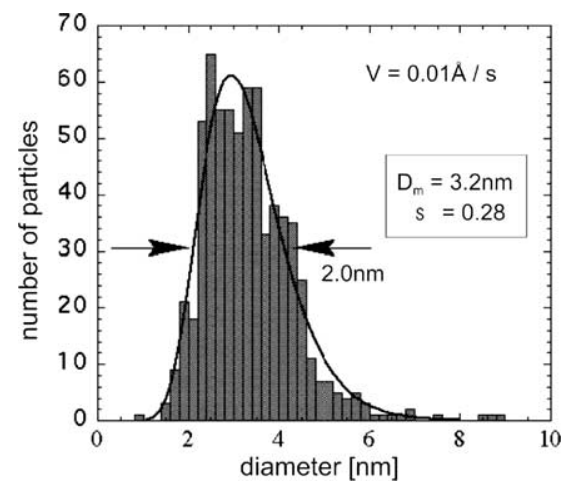

Fig. 46. Characteristic example of a size distribution of deposited mixed CoAg clusters derived from TEM observations. The solid line corresponds to the best fit of the distribution obtained with a log-normal function to deduce the mean cluster diameter $D_{\mathrm{m}}$ and the dispersion $\sigma[203]$.

Table 5

Numbers of atoms located at the cluster surface calculated for a series of perfect fcc-truncated octahedrons

\begin{tabular}{lll}
\hline $\begin{array}{l}\text { Number of atoms per cluster, } \\
\text { mean diameter, } m=\text { number } \\
\text { of shells }\end{array}$ & $\begin{array}{l}\text { Number of atoms in the first surface } \\
\text { monolayer (\% of the total number } \\
\text { of atoms in the cluster) }\end{array}$ & $\begin{array}{l}\text { Number of atoms in the two first } \\
\text { surface monolayer (\% of the total } \\
\text { number of atoms in the cluster) }\end{array}$ \\
\hline 201 atoms, $1.6 \mathrm{~nm}, m=3$ & 60.7 & 90.5 \\
586 atoms, $2.4 \mathrm{~nm}, m=4$ & 46.4 & 76.1 \\
1289 atoms, $3.3 \mathrm{~nm}, m=5$ & 37.4 & 64.4 \\
2406 atoms, $4 \mathrm{~nm}, m=6$ & 31.3 & 55.5 \\
\hline
\end{tabular}

are directly embedded in the niobium film of the microSQUID loop using a co-deposition technique in UHV in order to improve the cluster/SQUID magnetic coupling. A characteristic example of the 2D magnetization reversal diagram measured for one isolated CoAg cluster is shown in Fig. 47. From the fits of this experimental diagram using the method described in [204], we observe for some nanoparticles the

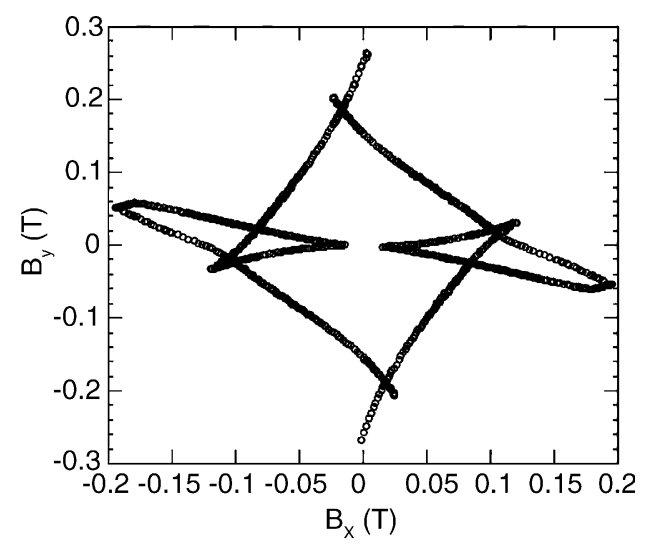

Fig. 47. Two-dimensional magnetic switching field distribution of an individual mixed CoAg cluster measured in the microSQUID plane at $35 \mathrm{mK}$ [204]. 
appearance of the fourth-order anisotropy energy term indicating that the magneto-crystalline anisotropy contribution dominates in this case [204]. By assuming that the Ag atoms segregate at the mixed cluster surface (in the case of large clusters) preventing the direct contact between the magnetic Co core of the cluster and the surrounding $\mathrm{Nb}$ matrix, a complex $\mathrm{Co}-\mathrm{Ag}-\mathrm{Nb}$ interface has to be considered instead of the more simple $\mathrm{Co} / \mathrm{Nb}$ one previously considered in the case of pure Co clusters embedded in niobium [204]. In this novel case, the Néel constant $\left(L \approx+1.5 \mathrm{MJ} / \mathrm{m}^{3}\right)$ is derived from magnetic measurements on Co-Ag multilayers [205]. Such a positive small $L$-value leads to a compensation of the shape and surface anisotropy terms and then the second-order anisotropy energy terms directly related to these contributions vanish. However, such a complete Ag-segregation probably does not take place in all CoAg clusters and further studies to really control the mixed cluster morphology are necessary, especially with various $\mathrm{Co}_{x} \mathrm{Ag}_{1-x}$ compositions.

Another type of mixed clusters studied is the Co-Pt system because a hybridization between the $3 \mathrm{~d}$ Co and the $5 \mathrm{~d}$ Pt-orbitals is expected in this case. The Co-Pt alloy formation at nanosize scale would lead to a high magnetization and an increase of the magnetic anisotropy as previously observed in the case of pure Co clusters embedded in a Pt matrix [206]. From an experimental point of view, the use of a nanosecondpulsed laser associated with a continuous inert gas flow has been found necessary to conserve the target composition in the produced mixed Co-Pt clusters, as reported earlier in the case of the Co-Ag system. TEM observations on Co-Pt clusters deposited on amorphous carbon coated grids at room temperature show crystallized nanoparticles with a size distribution centred around 2-3 nm. First conventional SQUID magnetometry measurements performed on collections of isolated Co-Pt clusters embedded in niobium films reveal a magnetic blocking temperature $T_{\mathrm{B}} \approx 50 \mathrm{~K}$ for mixed clusters containing a few hundreds Co atoms. As a comparison, note that this $T_{\mathrm{B}}$-value is intermediate between the values of 12 and $87 \mathrm{~K}$ reported previously for pure Co clusters embedded in a $\mathrm{Nb}$ matrix and a $\mathrm{Pt}$ matrix, respectively [207]. However, one has to remark that these pure Co clusters mentioned earlier with sizes of the order of $3 \mathrm{~nm}$ contain about one thousand Co atoms while the mixed Co-Pt ones contain only half this number. Consequently, the mixed Co-Pt system seems promising for the preparation of high anisotropy magnetic nanoparticles. As mentioned in the case of the $\mathrm{Co}-\mathrm{Ag}$ system, an optimization of the cluster composition $\left(\mathrm{Co}_{x} \mathrm{Pt}_{1-x}\right)$ will be investigated in a near future to improve magnetic characteristics. XPS measurements on discontinuous CoPt thin films are in progress in order to describe the electronic structure and the induced charge transfer effects due to the hybridization between both Co and Pt transition metals.

\subsection{Spin moments, orbital moments, and magnetic anisotropy of Co-Rh clusters}

Binary metallic clusters formed with a magnetic $3 \mathrm{~d}$ transition metal and a $4 \mathrm{~d}$ element, which is nonmagnetic in bulk but with a strong magnetic susceptibility like Pd or a weak, non-saturated magnetism like $\mathrm{Rh}$, could be an effective way to combine large magnetic moments with large magnetic anisotropy energy due to the large spin-orbit coupling of the $4 \mathrm{~d}$ element. The purpose of this section is to present theoretical results on the spin moments, orbital moments and on the magnetic anisotropy energy for several binary metallic nanostructures of $\mathrm{Co}-\mathrm{Rh}$ clusters. These specific systems are considered as representative of binary clusters. In particular, the role of structure, size and composition on the magnetic properties is quantified. For small clusters, results are presented for structure, spin moments and Co composition of $\mathrm{Co}-\mathrm{Rh}$ by using first principles methods. In the case of large clusters, the theory outlined in Section 3 was used for the calculation of the spin moments, orbital moments and magnetic anisotropy energy. The results are discussed in the context of available experimental results. Recently, Co-Rh 
nanoparticles (1-2 $\mathrm{nm}$ ) have been synthesized by decomposition of organometallic precursors in mild conditions of pressure and temperature in the presence of a polymer [110]. The results of the magnetic properties showed that the average magnetic moment per Co atom (i.e. assuming that Rh atoms carry vanishing magnetic moments) is about $2.38 \mu_{\mathrm{B}}$ for a Co concentration $x_{\mathrm{Co}} \simeq 0.5$. This value is much larger than the average magnetic moment in bulk alloys of similar concentration.

\subsubsection{First principles calculations of structure and spin moments of small $\mathrm{Co}_{N} R h_{M}$ clusters}

The interplay between structural, chemical and magnetic properties of small free $\mathrm{Co}_{M} \mathrm{Rh}_{N}$ $(N+M \leq 13)$ have been studied by Dennler et al. [208,209]. Calculations of the cohesive energy, structure and spin moments have been performed by solving the spin-polarized self-consistent KohnSham equations in the framework of a plane-wave basis treating core-electron effects within the projector-augmented wave (PAW) approximation [210,211]. All cluster structures were optimized by relaxing fully the atomic positions without symmetry constrains until the forces $F_{i}$ on each atom $i$ are vanishing (typically $\left|F_{i}\right| \leq 0.01-0.02 \mathrm{eV}$ ). This was done for each relevant value of the $z$-component of the total spin moment $S_{z}$. In this way, the interplay between magnetism and ground-state structure was quantified. Several initial geometrical configurations corresponding to different cluster topologies were considered in order to detect nearby isomers.

Representative results [208,209] for $\mathrm{Co}_{M} \mathrm{Rh}_{N}$ with $N+M=7$ atoms are presented in Fig. 48. One observes a monotonous decrease of the cohesive energy as we go from the pure $\mathrm{Rh}_{7}$ to pure $\mathrm{Co}_{7}$, in agreement with the lower cohesive energy of bulk Co compared to bulk Rh. The most stable structures are in general three dimensional $(N \geq 4)$ with planar isomers found at nearby energies. All studied Co-Rh clusters are magnetic with average spin moment per atom $\mu$ and local spin moments $\mu(i)$ that are often a factor of 2 larger than those of macroscopic crystals or alloys with similar concentrations. Moreover, one observes that $\mu$ and $\mu(i)$ tend to increase with increasing fraction of Co atoms. In some cases, the replacement of just one $\mathrm{Rh}$ atom by $\mathrm{Co}$ can lead to a remarkable global spin polarization and enhancement of the total cluster moment, which goes well beyond the individual contribution of the replaced atom.

From a local standpoint, one observes that the magnetic moments of Co atoms are not significantly affected by the Rh concentration even if the latter is increased beyond 50\%. The presence of Rh does not reduce the Co moments, which contrasts with the important reduction of Co moments observed in some macroscopic Co-Rh alloys. This is probably a consequence of the extremely reduced coordination
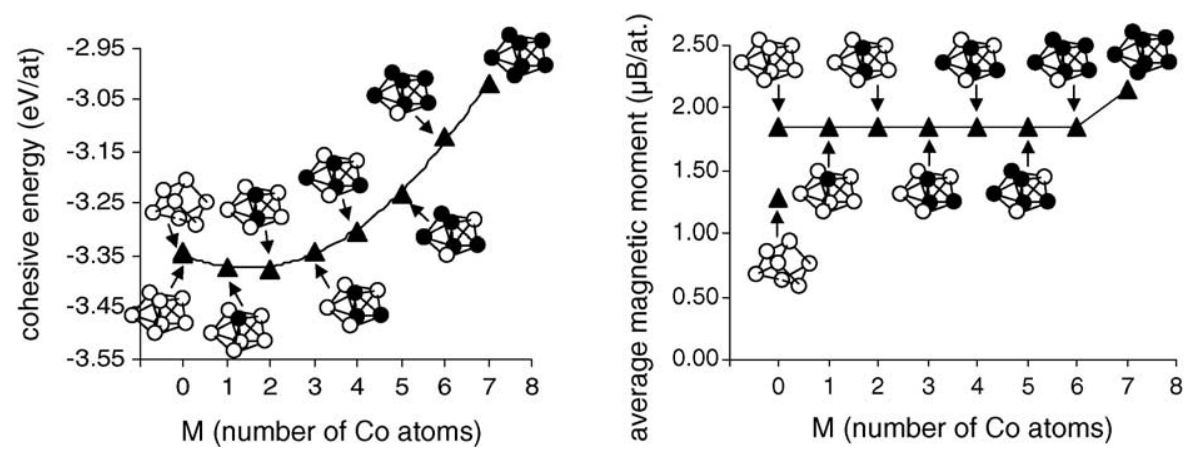

Fig. 48. Left: cohesive energy per atom $E_{\text {coh }}$ in eV. Right: average magnetic moment of $\operatorname{Co}_{M} \mathrm{Rh}_{N}$ clusters with $M+N=7$ as a function of Co concentration [208,209]. 
Table 6

Average magnetic moments (in $\mu_{\mathrm{B}}$ ) and lowest-energy magnetization direction $\delta$ (easy axis) of Co-Rh clusters

\begin{tabular}{|c|c|c|c|c|c|}
\hline Cluster & $\langle M\rangle_{\mathrm{T}} /(N+M)$ & $\langle M\rangle_{\mathrm{T}} / N$ & $\langle L\rangle_{\mathrm{T}} /(N+M)$ & $\langle L\rangle_{\mathrm{Rh}} / 2\langle S\rangle_{\mathrm{Rh}}$ & $\delta$ \\
\hline $\mathrm{Co}_{19}$ & 2.02 & 2.02 & 0.33 & & $x y$ \\
\hline $\mathrm{Co}_{43}$ & 1.98 & 1.98 & 0.29 & & $x$ \\
\hline $\mathrm{Co}_{19} \mathrm{Rh}_{24}$ & 0.80 & 1.44 & 0.06 & 0.07 & $z$ \\
\hline $\mathrm{Rh}_{19} \mathrm{Co}_{24}$ & 1.40 & 2.51 & 0.30 & 0.14 & $x$ \\
\hline $\mathrm{Rh}_{19} \mathrm{Co}_{60}$ & 1.65 & 2.17 & 0.26 & 0.09 & $z$ \\
\hline $\mathrm{Rh}_{19} \mathrm{Co}_{68}$ & 1.76 & 2.25 & 0.35 & 0.08 & $x$ \\
\hline $\mathrm{Rh}_{19} \mathrm{Co}_{66}$ & 1.71 & 2.20 & 0.30 & 0.10 & $x y$ \\
\hline $\mathrm{Rh}_{139} \mathrm{Co}_{182}$ & 1.18 & 2.08 & 0.19 & 0.07 & $z$ \\
\hline
\end{tabular}

For given $N$ and $M, \mathrm{Co}_{N} \mathrm{Rh}_{M}$ refers to an fcc-like cluster with a $\mathrm{Co}_{N}$ core covered by $M \mathrm{Rh}$ atoms, while in $\mathrm{Rh}_{M} \mathrm{Co}_{N}$ the core is Rh and the outer shells are Co. $\delta=z$ is a principal $\mathrm{C}_{4}$ symmetry axis of the cluster, $\delta=x$ is perpendicular to $z$ and along a nearestneighbour bond, and $\delta=x y$ points from the central atom to one of the vertices in the $x y$-plane.

number in these small clusters. From the point of view of $\mathrm{Rh}$, the presence of Co atoms in the cluster results in a remarkable increase of the local moments and in a larger stability of magnetism. From these results one concludes that small Co-Rh clusters are magnetic with average magnetic moments per atom that are significantly larger than those of bulk alloys of similar concentrations. For a given cluster size, $\mu$ increases with increasing $x_{\mathrm{Co}}$. The most stable isomers are usually not the most magnetic ones.

\subsubsection{Spin moments, orbital moments and magnetic anisotropy energy of large $C_{N} R h_{M}$ clusters}

Munoz-Navia et al. [212] calculated the spin moments, orbital moments and magnetic anisotropy energy of large $\mathrm{Co}_{M} \mathrm{Rh}_{N}$ clusters. The specific examples presented in the following are representative of a much larger of available calculations on binary clusters [212].

In Table 6, results are given for the magnetic moments and MAE of $\mathrm{Co}_{N} \mathrm{Rh}_{M}$. An fcc-like spherical cluster with $N+M=43$ atoms is considered as a representative example, which is formed by a central atom and its successive shells of NNs. This corresponds to a $\mathrm{Co}_{19}\left(\mathrm{Rh}_{19}\right)$ core covered with a $\mathrm{Rh}_{24}\left(\mathrm{Co}_{24}\right)$ shell. The relevant directions of the magnetization are $\delta=z$ which is a principal $\mathrm{C}_{4}$ symmetry axis of the cluster, $\delta=x$ which points along a NN bond perpendicular to $z$, and $\delta=x y$ which connects the central atom with one of the vertices in the $x y$-plane. For each considered cluster, the lowest energy direction or easy axis is indicated. For the sake of comparison, results also shown for $\mathrm{Co}_{19}$ and $\mathrm{Co}_{43}$. As expected, the total magnetic moment per atom $\langle M\rangle_{\mathrm{T}} /(N+M)$ decreases with decreasing Co concentration $x_{\mathrm{Co}}=$ $N /(N+M)$ (i.e. increasing $\mathrm{Rh}$ concentration). For example, $\langle M\rangle_{\mathrm{T}} /(N+M)=1.98 \mu_{\mathrm{B}}$ in $\mathrm{Co}_{43}$ $\left(x_{\mathrm{Co}}=1.0\right),\langle M\rangle_{\mathrm{T}} /(N+M)=1.40 \mu_{\mathrm{B}}$ in $\mathrm{Rh}_{19} \mathrm{Co}_{24}\left(x_{\mathrm{Co}}=0.56\right.$ with a $\mathrm{Rh}_{19}$ core $)$, and $\langle M\rangle_{\mathrm{T}} /(N+$ $M)=0.80 \mu_{\mathrm{B}}$ in $\mathrm{Co}_{19} \mathrm{Rh}_{24}\left(x_{\mathrm{Co}}=0.44\right.$ with a $\mathrm{Co}_{19}$ core $)$. Notice the particularly small value of $\langle M\rangle_{\mathrm{T}}$ in the case of a Co core, in contrast to the clusters with $\mathrm{Rh}$ core which still preserve a significant magnetization. This is mainly due to two effects: the larger NN bond length in the Rh core, which enhances the magnetic moments particularly at $\mathrm{Rh}$ atoms, and the orbital contribution $\langle L\rangle_{\mathrm{Co}} / N=$ $0.47 \mu_{\mathrm{B}}$ of the Co surface atoms. The importance of the induced magnetic moments at the Rh atoms is more clearly seen by considering, as in [110], the average total magnetic moment per Co atom, which is given by $\langle M\rangle_{\mathrm{T}} / N$. In the previous 43-atom examples, one has $\langle M\rangle_{\mathrm{T}} / N=1.98 \mu_{\mathrm{B}}$ for $x_{\mathrm{Co}}=1.00$ and $\langle M\rangle_{\mathrm{T}} / N=2.51 \mu_{\mathrm{B}}$ for $x_{\mathrm{Co}}=0.56$, which indicates that the $\mathrm{Rh}$ contribution is significant $\left[\langle M\rangle_{\mathrm{Rh}} / M \simeq 0.68 \mu_{\mathrm{B}}\right.$ in $\left.\mathrm{Rh}_{19} \mathrm{Co}_{24}\right]$. The fact that $\langle M\rangle_{\mathrm{T}} / N$ increases with decreasing $x_{\mathrm{Co}}$ shows that, upon alloying with $\mathrm{Rh}$, the Co atoms preserve the largest part of the magnetic moments they have in the 
pure Co case and that the induced moments at the Rh atoms are significant enough to override an eventual reduction of the Co contributions. A similar behaviour has been found in spin density functional calculations on small clusters [209].

Recent experimental results on the $\mathrm{NN}$ bond lengths and magnetic moments in Co-Rh clusters $[110,213]$ and the comparison with available theoretical calculations suggest that these binary clusters are likely to have a Rh core with Co rich outer layers. In fact, the measurements of NN bond lengths yield values that are very similar to that of bulk $\mathrm{Rh}$ [213]. Moreover, the measured magnetic moments per Co atom in the size range $N+M=200-400$ is $\langle M\rangle_{\mathrm{T}}^{\mathrm{expt}} / N \simeq 2.38 \mu_{\mathrm{B}}$ for $x_{\mathrm{Co}} \simeq 0.5$ [110], which are much larger than the theoretical values obtained for a Co rich core but which are consistent with the calculations for a Rh-rich core eventually with some degree of mixing at the interface. Furthermore, there is direct experimental evidence for the existence of an induced moment at the $\mathrm{Rh}$ atoms as observed in $\mathrm{X}$-ray magnetic circular dichroism measurements [214]. This is in qualitative agreement with the orbital-tospin ratios obtained in our calculations which amount to $\langle L\rangle_{\mathrm{Rh}} / 2\langle S\rangle_{\mathrm{Rh}}=0.07-0.14$ for $N+M=43$. Results on larger clusters in the experimentally relevant size range $(N+M \simeq 300$ atoms) confirm these conclusions [212].

The effects of structure and shape have been investigated by considering spherical-like and octahedral fcc clusters having $N+M=79,85$ and 87 atoms (see Table 6 and Fig. 49). In these cases, the dependence of the magnetic moments on Co concentration is qualitatively similar to what has been already discussed for smaller sizes. Comparing the spherical-like clusters, one observes somewhat larger $\langle S\rangle_{\mathrm{T}}$ and $\langle L\rangle_{\mathrm{T}}$ for $N+M=87$ than for $N+M=79$. This is mainly due to the larger orbital-moment contribution of the low-coordinated Co surface atoms which constitute the outermost shell for $N+M=$ 87 (see Fig. 49a and b). The same kind of local enhancement of $\left\langle L_{i \delta}\right\rangle$ is also found at the corners of the octahedral structure. Interesting symmetry effects are observed by comparing the MAE of spherical and octahedral clusters. In particular, in the latter case, full vectorial calculations as a function of both polar and azimuthal magnetization angles reveal a rich magneto-anisotropic energy surface with an easy axis $\delta=x y$ along the diagonal of the $x y$ square [212].

The environment dependence of the local moments provides further insight on the magnetic behaviour and on the proximity effects resulting from alloying and interface mixing [212]. Fig. 50 shows results for $\left\langle L_{j z}\right\rangle, 2\left\langle S_{j z}\right\rangle$, and $\left\langle M_{j z}\right\rangle=2\left\langle S_{j z}\right\rangle+\left\langle L_{j z}\right\rangle \cdot\left\langle L_{\delta}\right\rangle(j)$ in an fcc spherical-like cluster which is formed by a $\mathrm{Rh}_{79}$ core surrounded by surface Co shells including some degree of mixing at the Co-Rh interface. One observes that both $\left\langle L_{j z}\right\rangle$ and $2\left\langle S_{j z}\right\rangle$ generally increases with $j$, showing some oscillations as we move from the center to the surface of the cluster. Notice that the magnetic moments at the Rh atoms at the center of the cluster are

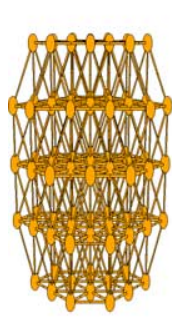

(a)

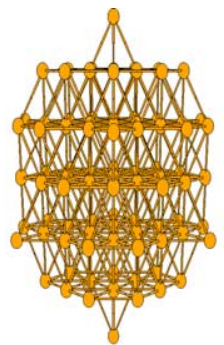

(b)

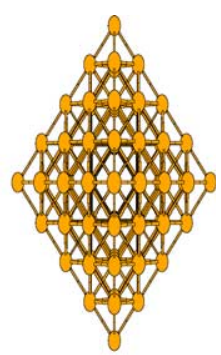

(c)

Fig. 49. Illustration of representative geometries considered in the calculations on $\mathrm{Co}_{N} \mathrm{Rh}_{M}$ : (a) spherical-like fcc with $N+M=79$; (b) spherical-like fcc with $N+M=87$; (c) octahedral fcc with $N+M=85$. 


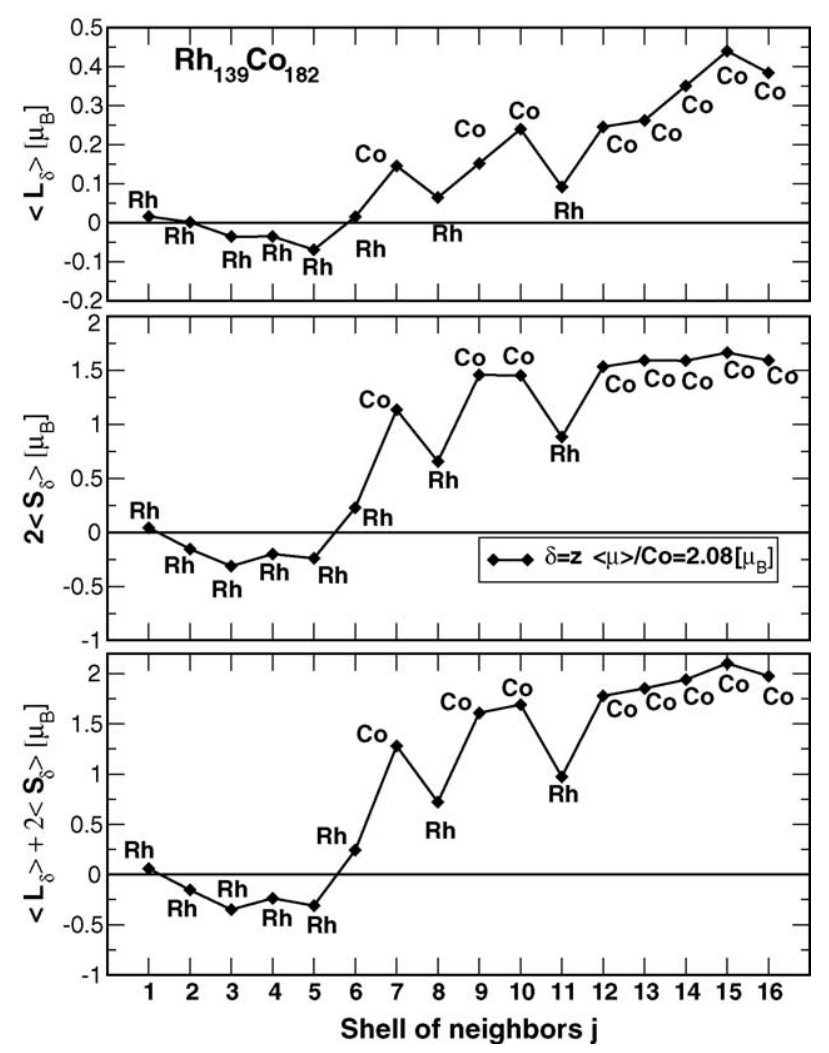

Fig. 50. Local magnetic moments in fcc-like $\mathrm{Co}_{139} \mathrm{Rh}_{182}$ : orbital contribution $\left\langle L_{j z}\right\rangle$, spin contribution $2\left\langle S_{j z}\right\rangle$ and total moment $\left\langle M_{j z}\right\rangle=2\left\langle S_{j z}\right\rangle+\left\langle L_{j z}\right\rangle$. The cluster is formed by a Rh core surrounded by surface Co shells including some degree of mixing at the Co- Rh interface. The chemical composition, which is assumed to the the same within the shell, is indicated for each $j$. The results refer to the average at each $\mathrm{NN}$ shell $j$ ordered by increasing distance to the central atom $j=1$. The magnetization direction is along the easy axis $\delta=z$. The lines are a guide to the eye.

antiparallel to the majority spins. This tendency to an antiferromagnetic-like order is characteristic of systems showing weak magnetism. A similar behaviour is observed in pure $\mathrm{Rh}_{M}$ clusters across the sizedependent transition from a ferromagnetic to a paramagnetic state which occurs for $M \approx 50$ [96]. On the other side, particularly large positive values of $\left\langle L_{j z}\right\rangle$ and $2\left\langle S_{j z}\right\rangle$ are found are the interface Rh atoms (e.g. $j=8$ and $j=11$ ). As a result the average Rh contribution to $\langle M\rangle_{\mathrm{T}}$ is positive. For these sizes it amounts to about $20 \%$ of the total cluster moment and is thus responsible for the experimentally observed enhancement of the average moment per Co atom $\left[\langle M\rangle_{\mathrm{Rh}} / M \simeq 0.25 \mu_{\mathrm{B}}\right.$ for $\left.N+M=321\right]$. These calculations show the dominant role of the $\mathrm{Co}-\mathrm{Rh}$ interfaces and of the resulting proximity effects.

Alloy clusters open interesting possibilities of tailoring the magnetic behaviour as a function of size and relative concentrations. As already discussed, the magnetic moment per Co atom increases for given size when Co atoms are replaced by $\mathrm{Rh}$. However, except for the very small sizes $(M \leq 50)$ where $\mathrm{Rh}_{M}$ is magnetic on its own, one observes that $\langle M\rangle_{\mathrm{T}} / N$ in $\mathrm{Co}_{N} \mathrm{Rh}_{M}$ starts to decreases if $x_{\mathrm{Co}}=N /(N+M)$ is reduced beyond a certain threshold. In this Rh-rich limit, the Co content in the cluster is not large enough to stabilize a significant net magnetization of the Rh atoms and ferromagnetic order breaks down. Let us recall that pure $\mathrm{Rh}_{M}$ is not magnetic in the experimentally relevant size range of about 300 atoms. In this 
case, $\langle M\rangle_{\mathrm{T}} / N$ shows a maximum as a function of $x_{\mathrm{Co}}$. Actually, a similar situation is known to occur in bulk CoRh alloys. However, recent calculations [212] on alloy clusters reveal an interesting new sizedependent effect which shifts the maximum in magnetic moment from $x_{\mathrm{Co}} \simeq 0.75$ in the bulk to $x_{\mathrm{Co}} \simeq 0.5$ at least for $N+M \leq 500$. All magnetic properties are affected by this non-monotonous behaviour. In particular, the MAE follows in general a similar trend and is thus susceptible of being optimized as a function of composition, which has an obvious importance for potential technological applications.

To summarize this section, we have shown first results on different types of binary clusters, softmagnetic FeCo clusters as well as clusters containing Co as a $3 \mathrm{~d}$ elements in contact with Pt, Ag, Sm or $\mathrm{Rh}$ in order to enhance the magneto-crystalline anisotropy energy. The chemical composition in the clusters strongly depend on the operation parameters of the cluster sources even in the case of bulk alloy target which opens the possibility to vary the composition over a certain range by using specific parameters of the cluster sources. All of these binary cluster materials show a variety of interesting properties as a function size, structure and composition, which deserve further systematic experimental and theoretical studies. The existence of ordered phases and/or core-shell structures play dominant roles in the magnetic behaviour. The XMCD technique opens the possibility to address the individual chemical elements in such alloys and to study their magnetic properties (e.g. orbital and spin moments, preferential axis of magnetization). Remarkable surface and interface effects are also expected for other $3 \mathrm{~d}-4 \mathrm{~d}$ finitesize alloys. From the theoretical point of view Co-Pd clusters appear to be very promising, particularly concerning magnetic anisotropy, since the Co-Pd interfaces have already revealed remarkable effect in the context of bulk alloys, thin films, and deposited clusters.

\section{Self-organized Fe and Co clusters on $\operatorname{Au}\left(\begin{array}{lll}1 & 1 & 1\end{array}\right)$}

The magnetic properties of self organized clusters on the $\mathrm{Au}\left(\begin{array}{lll}1 & 1 & 1\end{array}\right)$ surface, from isolated islands to the $2 \mathrm{D}$ limit, will be revised in this section and experimental results will be presented. The goal is to develop a good understanding of self-organized magnetic structures in terms of their basic parameters such as cluster size and density. We will discuss the magnetic properties of nanostructures, from non-interacting well separated islands, to interconnected ones forming stripes, to almost continuous films of two-dimensional character. Ultimately, magnetism will be viewed as the result of interacting building blocks (spin blocks); a particularly fruitful approach in the case of self-organized cobalt dots. In the following, we will analyze several examples on how ferromagnetism sets in as a function of coverage. We will limit ourselves to welldefined and characterized structures from both magnetic and topographic point of views.

\subsection{Assembly of perpendicularly magnetized clusters on a regular network}

As mentioned earlier, Co grows in well ordered clusters of bilayer height on the surface zigzag reconstruction of $\mathrm{Au}\left(\begin{array}{lll}1 & 1 & 1\end{array}\right)$ (see Fig. 11). One expects the formation of separate and magnetically noninteracting clusters in the early stage of growth. Then, they expand laterally as a function of coverage, until they come close to contact at about 1.0 ML (since the clusters are bilayers, this means that the gold surface is half-covered by Co). For coverages below 1.0 ML, the assumption of non, or weakly interacting clusters holds. Assemblies of small ferromagnetic clusters often show an average zero magnetization because of thermal fluctuations. The clusters behave superparamagnetic like independent giant magnetic moments, fluctuating on a very short time scale. The fluctuations can be blocked only at 


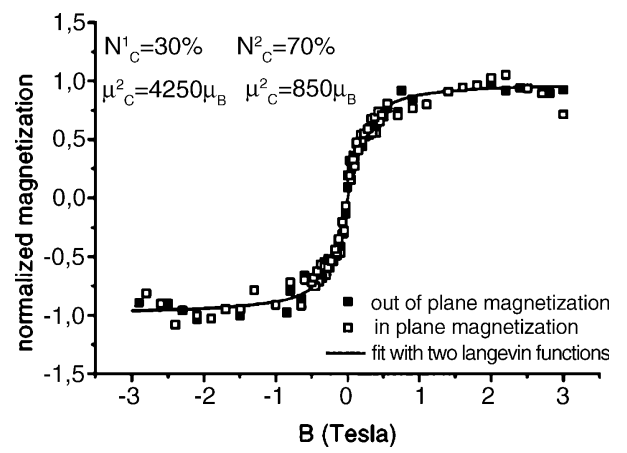

Fig. 51. SQUID magnetization curves recorded a $290 \mathrm{~K}$ for $0.4 \mathrm{ML}$ Co on Au(l 111$)$. The data points are fitted by a sum of two Langevin functions corresponding to two populations of clusters with different sizes (given in values of giant moments $\mu_{\mathrm{c}}^{i}$ ). The fit gives the proportion of the two populations, $N_{\mathrm{c}}^{i}[178]$.

very low temperatures and saturated in high fields. In situ Kerr effect measurements on as-grown clusters are difficult since the saturation fields needed to evidence the superparamagnetic state are high. Therefore, the samples have to be covered by a protective film to be measured ex situ in high fields by Kerr effect or SQUID. A hysteresis curve, characteristic of a superparamagnetic state, is then obtained (Fig. 51) [178,215,216]. The saturation fields are in the order of $1 \mathrm{~T}$ or $10 \mathrm{kOe}$, respectively. Since the magnetization of superparamagnetic clusters is described by a Langevin function of argument $N \mu_{\mathrm{B}} / k_{\mathrm{B}} T$, the experimental curve allows one, in principle, to determine the size of the clusters. Often it is assume that the clusters are all of the same size. This is actually the case for the self-organized Co clusters nucleated on the herringbone reconstruction. However, the magnetization curves in $[178,215]$ are measured on Co clusters deposited on thick gold films grown on mica substrates. The Au surface of such samples has much more defects than the one of a well prepared $\mathrm{Au}\left(\begin{array}{lll}1 & 1 & 1\end{array}\right)$ single crystalline surface. On the $\mathrm{Au} / \mathrm{mica}$ substrates, regions with the usual herringbone reconstruction coexist with linearly reconstructed ones, where the Co atoms nucleate randomly and form clusters, four to five times larger than those nucleated on the herringbone [178]. Although they represent only 20-30\% of the total number of clusters, they contribute to the main part of the magnetic signal due to the fact that the clusters are much larger. Modifications in the electronic structure are expected in small cobalt clusters on $\mathrm{Au}\left(\begin{array}{lll}1 & 1 & 1\end{array}\right)$. In particular, an enhanced orbital magnetic moment has been evidenced by X-ray magnetic circular dichroism. The enhanced orbital contribution leads to a slightly larger anisotropy in the small clusters [5].

\subsection{The effect of cluster coalescence: $\mathrm{Fe} / \mathrm{Au}\left(\begin{array}{lll}1 & 1 & 1\end{array}\right)$}

Strain and edge effects upon coalescence of islands have been studied at self-organized Fe clusters on $\mathrm{Au}\left(\begin{array}{lll}1 & 1 & 1\end{array}\right)$. The STM study presented in Fig. 52 shows that the coalescence of clusters into stripes occurs at $0.3 \mathrm{ML}$ [217]. On the verge of coalescence, unusual magnetic properties have been measured by X-ray magnetic circular dichroism [183]. As shown in the right part of Fig. 52, a quenching of the orbital magnetic moment is first observed, as the dimensionality of the system increases. Simultaneously, the spin moment (given per valence hole $n_{\mathrm{h}}$ ) is found to increase from $0.44 \mu_{\mathrm{B}} / n_{\mathrm{h}}$ up to $0.71 \mu_{\mathrm{B}} / n_{\mathrm{h}}$ (using $n_{\mathrm{h}}=3.39$, these values are identical to $1.5 \mu_{\mathrm{B}}$ per atom up to $2.4 \mu_{\mathrm{B}}$ per atom, respectively). This behaviour is due to the fact that upon coalescence, the clusters change from a contracted lattice parameter with respect to the $\mathrm{Au}$ substrate, to the new pseudomorphic lattice parameter of the gold. During 

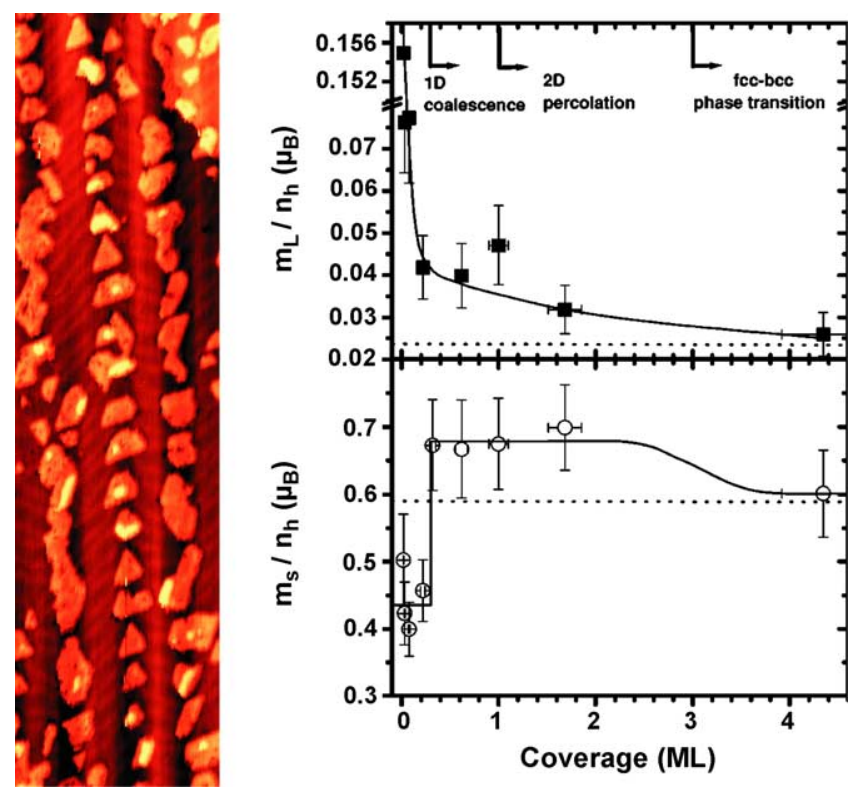

Fig. 52. Left part: STM image of the cluster coalescence at 0.32 ML Fe deposited at $300 \mathrm{~K}$ (image: $130 \mathrm{~nm} \times 33 \mathrm{~nm}$ ); right part: XMCD data as a function of Fe coverage. Top: orbital moment $M_{\mathrm{L}}$ and bottom: spin moment $m_{\mathrm{S}}$ per valence hole $n_{\mathrm{h}}$ [183]. The dotted lines represent the bulk Fe values.

coalescence, the nearest-neighbour distance increases by $0.04 \AA$. Furthermore, the XMCD measurements also show that the magnetization of iron clusters is in the plane of the surface while it goes out-ofplane upon coalescence. There is an on-going calculation in order to get a deeper insight into the spin reorientation transition of ultrathin films of $\mathrm{Fe}$ on $\mathrm{Au}\left(\begin{array}{lll}1 & 1 & 1\end{array}\right)$ as a function of coverage. A similar abrupt onset of ferromagnetic order has been observed on the Fe/W(llll 10$)$ system at $0.6 \mathrm{ML}$ coverage corresponding to the percolation of the randomly distributed Fe islands [218].

\subsection{Interacting islands; from $1 D$ chains to $2 D$ magnetism}

The case of Co clusters on $\mathrm{Au}\left(\begin{array}{lll}1 & 1 & 1\end{array}\right)$ has been analyzed in detail over a wide range of coverages. By increasing the amount of $\mathrm{Co}$, the bilayer clusters grow laterally and start to interact along $1 \mathrm{D}$ arrays in the direction where they are closest to each others until they finally coalesce for a coverage of $1 \mathrm{ML}(\Theta=1)$. They form more or less continuous bilayer chains, about $8 \mathrm{~nm}$ wide and $100 \mathrm{~nm}$ long (see left part of Fig. 55). The chains, which are separated approximately by $15 \mathrm{~nm}$, remain superparamagnetic. If these chain structures are considered as a unidimensional arrangement of spins with perpendicular anisotropy (1D Ising model), one does not expect any magnetic long range order. Indeed, the chains behave as large fluctuating spin blocks, whose sizes are larger than that of individual clusters. In this case, the magnetization curves are not supposed to fit a Langevin function, since the clusters are interacting with each other. When the amount of Co is further increased, adjacent chains start to connect through clusters growing in between the chains, so that finally a quasi continuous $2 \mathrm{D}$ film is formed at a coverage of $\Theta=2$. While the clusters with $N=200$ atoms are superparamagnetic as shown by SQUID magnetometry, with a blocking temperature below $30 \mathrm{~K}$, this blocking temperature increases to about 
$100 \mathrm{~K}$ for $N \approx 1200$ atoms. The formation of chains for $\Theta \geq 1$ induces an opening of the hysteresis loop at $300 \mathrm{~K}$ on the experimental time scales. However, the saturation field remains relatively high and is only accessible above $\Theta=1.5$ in the in situ experiment [219]. In order to describe the onset of ferromagnetism with Co coverage, Monte Carlo simulations were performed on a $N \times N$ hexagonal array of Co clusters of about $75 \AA$ average diameter ( 2 ML thick) with a giant magnetic moment. The system is described in a 2D Heisenberg-like model. The Hamiltonian is:

$$
H=\frac{\gamma}{4} \sum \delta_{i j} \sigma_{i} \varepsilon_{i} \sigma_{j} \varepsilon_{j}-K V \sum\left(\underset{i}{z} \varepsilon_{i}\right)^{2}-\mu_{0} M H \sum \underset{i}{z} \varepsilon_{i}
$$

where $\sigma_{i}$ is the normalized magnetization of the cluster at a site $i$ ( $M$ is the saturation magnetization), $\varepsilon_{i}=1$ or 0 if the site is occupied or not by a cluster, $\delta_{i j}=1$ for nearest-neighbour clusters ( 0 otherwise), $V$ the individual cluster volume, $K$ the perpendicular anisotropy constant, $H$ the external field (applied in the $z$-direction, perpendicular to the surface), $\gamma$ is the wall energy for two opposite magnetizations in two adjacent clusters. The wall energy can be estimated from a micromagnetic model $(\gamma=2 J K)$. The dipolar coupling between chains has been neglected in first approximation. As will be shown in a further work, its consideration leads to slightly smaller remnant fields. The numerical values used for the simulation are reported in the caption of Fig. 54. The calculation starts with 1 ML coverage, i.e. arrays of clusters separated by lines of unoccupied sites. Then, the unoccupied sites are randomly filled with clusters. The $\sigma^{z}$-maps (there is nearly no in-plane component) of $\sigma$ are shown for several coverages in the demagnetized state at $=300 \mathrm{~K}$. Below 1.5 ML (Fig. 54a and b), the up and down domains are small and confined within the chains; connections to the adjacent row are too scarce to allow a strong magnetic coupling. At about 1.5 ML, (Fig. 54c) the domain size increases and spread all over the whole surface. At $2 \mathrm{ML}$ (Fig. 54d), the domain structure is very similar to the one observed experimentally by secondary electron microscopy with polarization analysis [220]. The corresponding simulated magnetization curves are represented. One observes a drastic decrease of the saturation field from 1 to $1.5 \mathrm{ML}$. A significant remnant magnetization appears above 1.2 ML. These simulations are in quantitative agreement with the experimental results (cf. Fig. 53).
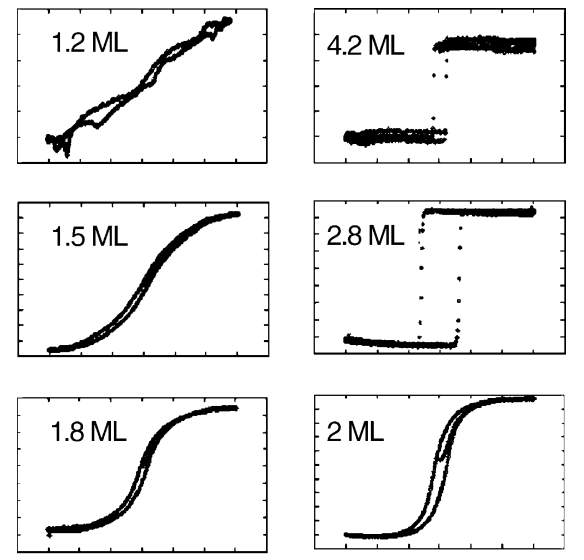

$\stackrel{500 \mathrm{Oe}}{\longrightarrow}$

Fig. 53. Hysteresis loops as a function of coverage $\Theta$, taken in the polar configuration of the MOKE. Below a coverage of $\Theta<1.5$ magnetic fields of $0.1 \mathrm{~T}$ are too small to saturate the sample [219]. 

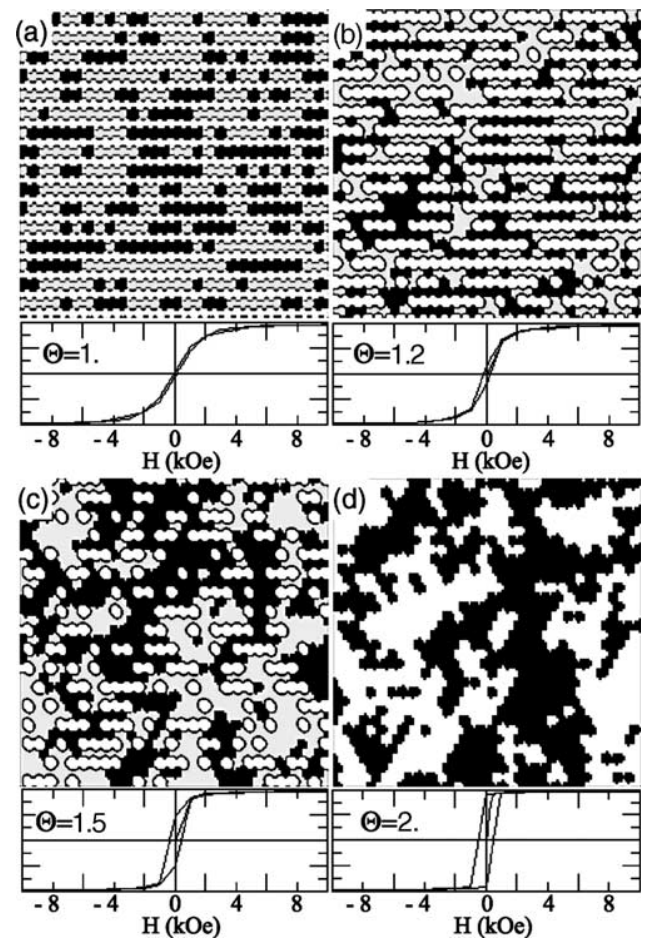

Fig. 54. Demagnetized $\sigma^{z}$ maps of $30 \times 30$ sites for different coverages $\Theta$. Unoccupied sites are in white, occupied sites with up (down) magnetization in grey (black). Hysteresis loops and first magnetization loops are shown. $\gamma S / k_{\mathrm{B}}=125 \mathrm{~K}$, $K V / k_{\mathrm{B}}=1000 \mathrm{~K}, M / k_{\mathrm{B}}=1000 \mathrm{~K} / \mathrm{T}[178]$.

\subsection{In-plane anisotropy induced by low temperature growth}

As was discussed previously, the growth of Co on $\mathrm{Au}\left(\begin{array}{lll}1 & 1 & 1\end{array}\right)$ is normally governed by the nucleation on point dislocations of the zigzag reconstruction. This particular growth mode of bilayer-high islands is observed down to temperature as low as $150 \mathrm{~K}$. At some point when lowering the substrate temperature, the mean free path of adatom diffusion becomes small compared to the separation between point dislocations of the zigzag reconstruction. The new islands density is then given from Eq. (2) by $N \approx R^{1 / 3} \exp \left(-E_{\mathrm{d}} / 3 k T\right)$, where $R$ is the deposition rate and $E_{\mathrm{d}}$ the diffusion barrier for a cobalt adatom on the gold surface. A much higher density of islands than the one anticipated from self-assembly is therefore achieved (see Fig. 55). Growth first proceeds by random nucleation of monolayer thick islands with lateral sizes of about $5 \AA$. The small monolayer thick islands then coalesce well before the critical size for bilayer islands is reached [221]. A quasi layer-by-layer, 2D growth, occurs contrary to the 3D growth observed at $300 \mathrm{~K}$. As can be foreseen, the properties of films grown at low and high temperatures will be quite different as will be illustrated now on the example of magnetism. From the magnetic point of view, the results are quite remarkable. Contrary to the films grown at $300 \mathrm{~K}$, which possess a perpendicular magnetization, the films obtained at $30 \mathrm{~K}$ are magnetized in the plane of the film (Fig. 56) [84]. The unprotected films (with $\theta \leq 5 \mathrm{ML}$ ) show a reorientation of the easy axis of 

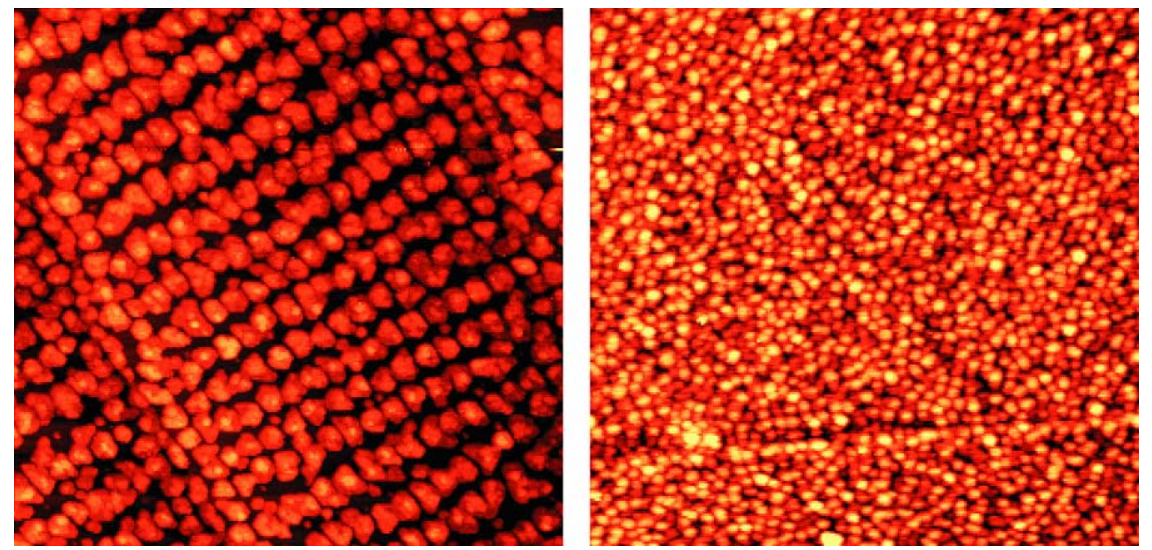

Fig. 55. Comparison between 1.3 ML of Co grown on $\mathrm{Au}\left(\begin{array}{lll}1 & 1 & 1\end{array}\right)$ at $300 \mathrm{~K}$ (left image with $\left.150 \mathrm{~nm} \times 150 \mathrm{~nm}\right) \mathrm{and} 1.0 \mathrm{ML}$ of Co grown on $\mathrm{Au}\left(\begin{array}{lll}1 & 1 & 1\end{array}\right)$ at $30 \mathrm{~K}$ (right image $100 \mathrm{~nm} \times 100 \mathrm{~nm}$ ). In the right image compact stacking of monolayer clusters with a diameter of $0.5 \mathrm{~nm}$ occurs while growth at $300 \mathrm{~K}$ (left part) leads to bilayer clusters of $7 \mathrm{~nm}$ each [84].

magnetization from parallel to perpendicular above $260 \mathrm{~K}$. We believe that structural changes occur upon annealing, modifying the magneto-elastic contribution to the anisotropy. The reorientation can be avoided by covering the $30 \mathrm{~K}$ grown Co films with $\mathrm{Au}$. These results open the way to spin engineering of cobalt films. For example, it becomes possible to tune the magnetic anisotropy from in-plane to out-ofplane by carefully choosing the annealing temperature. This last result is particularly important in view of renewed interest in tilted media for magnetic data storage applications [222]. The exact origin of the inplane anisotropy of Co when grown under low temperature conditions is not completely clear yet. It seems that low-temperature deposited $\mathrm{Co}$ on $\mathrm{Au}\left(\begin{array}{lll}1 & 1 & 1\end{array}\right)$ has a smaller magneto-crystalline anisotropy than Co grown under normal conditions and therefore the shape anisotropy can force the magnetization in-plane. It should be mentioned that an in-plane anisotropy is actually predicted by first principle calculations for an uncovered, pseudomorphic Co monolayer on $\mathrm{Au}\left(\begin{array}{lll}1 & 1 & 1\end{array}\right)$ [223]. However, the same calculation also predicts a reorientation of the easy axis for a gold capping of $1 \mathrm{ML}$. This last result disagrees with the experimental results since the gold coverage definitely stabilizes the in-plane magnetization.
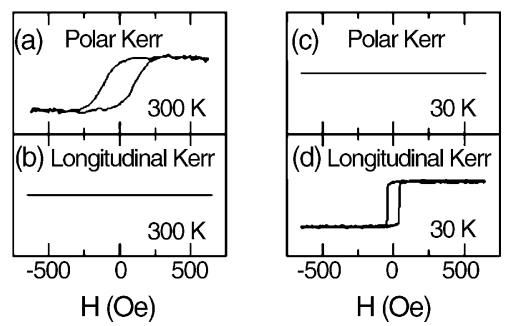

Fig. 56. Kerr signal in the polar and longitudinal configuration for films of $1.7 \mathrm{ML}$ of Co deposited at $300 \mathrm{~K}$, (a and b); deposited at $30 \mathrm{~K}$, (c and d) [84]. 


\subsection{Magnetic tuning through temperature induced segregation of binary clusters}

In the previous section, it was shown that that co-deposition of metal vapour onto a substrate held at low temperature provides an alternative approach to the synthesis of cluster assembled films. The advantage of this technique is that no cluster beam source is necessary. Furthermore, the low temperature deposition is a powerful way to force originally immiscible systems to form an alloy. In order to measure the efficiency of this technique, we choose the Co-Au system for its strong tendency to segregate. Contrary to bulk alloy materials, where diffusion is activated over high barriers the enhanced surface diffusion in thin films easily allows the tuning of properties over large ranges of parameters.

An intimate mixing of $\mathrm{Co}$ and Au clusters, about 20 atoms in size is obtained by co-deposition at $30 \mathrm{~K}$. Controlled segregation and growth by coalescence of larger Co clusters is then induced by annealing. In the following we will focus on a Co-concentration of $70 \%$ and an equivalent thickness of 3.5 ML. By increasing the temperature to $T=150 \mathrm{~K}$, a magnetic signal progressively appears in the polar configuration of the MOKE, the signal needs about $120 \mathrm{~min}$ to stabilize in a nice rectangular hysteresis loop with a coercive field of 60 Oe. The tiny clusters that make up the film start to merge into each others, thereby stabilizing the ferromagnetic phase. The magnetic behaviour is then dictated not only by the anisotropy of individual clusters but also by the interaction at various length scales through exchange and dipolar couplings. Annealing the cluster film to higher temperatures permits a fine tuning of the critical temperature $T_{\mathrm{c}}$ above which the film undergoes a transition from ferro- to paramagnetism. $T_{\mathrm{c}}$ can be adjusted from $200 \mathrm{~K}$ all the way up to $500 \mathrm{~K}$. The same technique allows the variation of the coercive field over two orders of magnitude, from a few Oe up to 500 Oe. In order to get a better insight into the segregation mechanism, in connection with magnetism, a Monte Carlo based simulation of a binary alloy was performed [224]. We performed Monte Carlo simulations on the phase separation process of a binary alloy and the corresponding evolution of the magnetic hysteresis. The goal of the simulation is to find the tendency of the $\mathrm{Au}-\mathrm{Co}$ solution to out-mix at a given temperature and correlate the alloy structure with the magnetic properties. In our model for the phase separation we consider a binary alloy $\mathrm{A}_{x} \mathrm{~B}_{y}$ with a certain concentration of vacancies $\left(c_{\mathrm{V}}=1-x-y\right)$ [225] on a two-dimensional triangular lattice. Repulsive pair interactions between nearest-neighbour AB pairs are assumed. The Hamiltonian of the alloy is:

$$
H=\sum_{\langle i, j\rangle}\left(C_{i}^{\mathrm{A}} C_{j}^{\mathrm{A}} \phi^{\mathrm{AA}}+C_{i}^{\mathrm{A}} C_{j}^{\mathrm{B}} \phi^{\mathrm{AB}}+C_{i}^{\mathrm{B}} C_{j}^{\mathrm{B}} \phi^{\mathrm{BB}}\right),
$$

where $C_{i}^{X}=1(0)$ if an atom $X$ exists (does not exist) in site $-i$ and $\phi^{X X^{\prime}}$ is the pair potential between atoms $X$ and $X^{\prime}$ that lie in nearest-neighbour sites. The Hamiltonian of the binary alloy with vacancies can be transformed to an Ising Hamiltonian with spin $S=1$ :

$$
H=-J_{\mathrm{a}} \sum_{\langle i, j\rangle} S_{i} S_{j}+H_{0},
$$

where $J_{\mathrm{a}}=\frac{1}{2}\left\{\phi^{\mathrm{AB}}-\frac{1}{2}\left(\phi^{\mathrm{AA}}-\phi^{\mathrm{BB}}\right)\right\}$ and $H_{0}$ is a constant. The three possible values of the spin $S_{i}=$ $+1,0,-1$ correspond to the site $-i$ being occupied by an atom A, a vacancy or an atom B, respectively. The dynamics associated to this model assumes a vacancy mechanism [226] with different jump rates $\left(\Gamma_{\mathrm{A}}, \Gamma_{\mathrm{B}}\right)$ for the atoms $\mathrm{A}$ and $\mathrm{B}$.

The magnetic properties of the annealed sample are modeled by a classical Heisenberg Hamiltonian with nearest-neighbour exchange interactions, long range dipolar interactions and perpendicular 

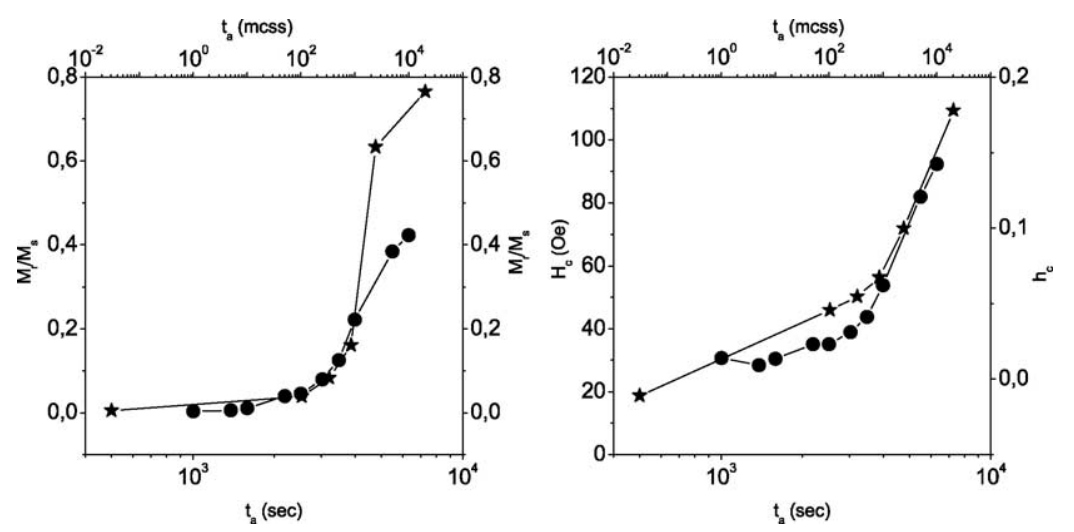

Fig. 57. Time evolution of the remanence (left) and coercivity (right) of a $\mathrm{Co}_{70} \mathrm{Au}_{30}$ film with $25 \%$ vacancies annealed at $150 \mathrm{~K}$. Stars: experimental data; circles: simulation data. The bottom and left axes in each graph correspond to experimental scales, while top and right axes correspond to simulation scales (mcss: time measured in Monte Carlo steps per spin) [224].

anisotropy described in Eq. (20) in Section 6.2.1. The evolution of the magnetization curve during annealing has been calculated, clearly showing a transition from superparamagnetism with no hysteresis, to ferromagnetism with an almost square loop. Fig. 57 shows the increase of $M_{\mathrm{s}}$ and $H_{\mathrm{c}}$ as a function of annealing time, in close agreement with the experimental data.

Simulations have been performed on a $50 \times 50$ triangular lattice with periodic boundaries. For the alloy parameters we have taken $\phi^{\mathrm{CoCo}}=-0.0837 \mathrm{eV}$ per atom, $\phi^{\mathrm{AuAu}}=-0.0212 \mathrm{eV}$ per atom and $\phi^{\mathrm{CoAu}}=-0.0276 \mathrm{eV}$ per atom as obtained from theoretical calculations [227]. The analysis of the grown samples gives an approximate vacancy concentration $c_{\mathrm{V}} \approx 25 \%$ which we have included in our simulations. The simulation starts from a homogeneous alloy $\mathrm{Co}_{70} \mathrm{Au}_{30}$ containing $25 \%$ vacant sites. Each Co site in the simulation cell corresponds to a Co cluster in the as-deposited samples. Snapshots of the evolution of the domains in a typical sample are shown in Fig. 58, where the growth of the Co domains with annealing "time" (measured in Monte Carlo steps per spin) is demonstrated. The vacancies tend to
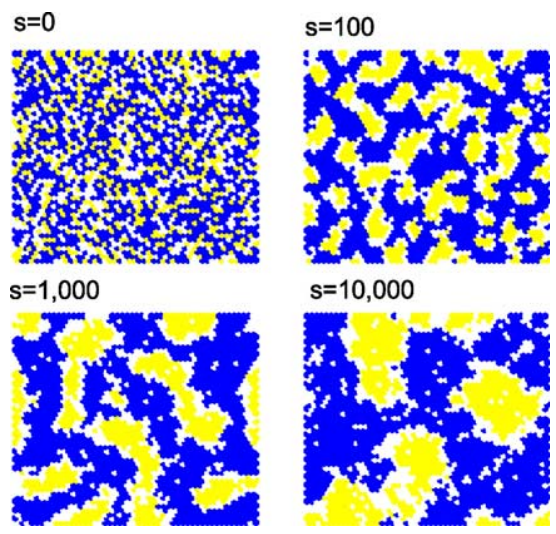

Fig. 58. Segregation of a $\mathrm{Co}_{70} \mathrm{Au}_{30}$ cluster film with $25 \%$ vacancies at $150 \mathrm{~K}$ as a function of the Monte Carlo steps (s). Dark correspond to Co atoms, light sites to Au atoms and white sites to vacancies [224]. 
aggregate in the interface between Co-rich and Au-rich regions, thus reducing the average size of the Co islands. Simulations have also been performed with different jump rates between $\mathrm{Co}$ and $\mathrm{Au}$, assumed to be proportional to their atomic masses $\left(\Gamma_{\mathrm{Au}} / \Gamma_{\mathrm{Co}} \approx 0.5\right)$. It has been observed that the net effect was to slow down slightly the phase separation without introducing any qualitative differences in the final morphology of the system.

Summarizing, we would like to mention that simultaneous analysis of STM topography and magnetic properties of organized islands on surfaces has conducted to an improved understanding of the interrelation between growth and magnetism of films and organized clusters. Specific properties of magnetic "films" appear more and more as a result of the spontaneous nanostructuring during growth (interaction between spin block, pinning of magnetic walls on island boundaries, etc.) and not as intrinsic properties of monolithic, continuous films as previously assumed. The recent development of variable temperature STM equipment allows the characterization of highly non-equilibrium materials with unexpected properties. This very fruitful approach also opens up new ways to exploit the atomic diffusion to tailor to a large extent the density and shapes of nanostructures. Although the feasibility of selforganization on dislocation networks has been demonstrated, many problems remain to be addressed. A major challenge in future work will consist in tuning the periodicity of templates for self-organization purposes since presently only a few combinations of lattice mismatch of hetero-epitaxial systems (see $\mathrm{Ag} / \mathrm{Pt}$ or $\mathrm{Au}\left(\begin{array}{lll}1 & 1 & 1\end{array}\right)$ ) are used. Ideally it should be possible to adjust continuously the periodicity from one value to another. Up to now, ordered, well separated structures could be synthesized on a few systems. The magnetic blocking temperatures thus achieved on Co dots on gold only amount to a few $10 \mathrm{~K}$. Blocking temperatures in excess of $300 \mathrm{~K}$ are reached only when structures start to interact magnetically. In order to increase the blocking temperature of each dot separately the volume of the dot must be increased, eventually its aspect ratio must be optimized.

\section{Conclusion and outlook}

This manuscript gives an overview on fundamental research related to magnetic clusters exposed on surfaces or embedded intro matrices. We have focussed mainly on metal clusters (pure $3 \mathrm{~d}$ metals and binary alloys) deposited from a cluster beam and 3d nanostructures grown on a $\mathrm{Au}\left(\begin{array}{lll}1 & 1 & 1\end{array}\right)$ surface by selforganization, the main results are summarized at the end of each chapter. The first part describes the production of nanoparticles in the gas phase using different types of cluster sources, e.g. a gas aggregation source for small metal cluster (less than $3 \mathrm{~nm}$ in diameter), an arc discharge cluster source, which is able to produce pure and alloy clusters with sizes from about 3 to $15 \mathrm{~nm}$, and a laser vaporization/gas condensation cluster source, which can produce binary clusters (typically in the size regime from 2 to $6 \mathrm{~nm}$ ) with different compositions. After an introduction into cluster magnetism from the theoretical point of view and experimental methods for characterizing magnetic properties, we have presented experimental results on exposed clusters on surfaces as well as in matrices for different size regimes. The investigations on pure cluster were mainly related to the orbital and spin moments using the technique of X-ray magnetic circular dichroism (XMCD) in photoabsorption. They clearly show that enhanced orbital moments are present also in deposited clusters over a wide size regime. For Fe and Co clusters in Ag matrices the magnetic behaviour has been shown between the extreme of isolated clusters behaving superparamagnetic and a correlated super-spin glass for volume fractions above the percolation threshold. 
We would like to point out that mass-selected binary alloy clusters are promising candidates for both future investigations on fundamental research as well as technical application. Binary alloy clusters from the magnetic $3 \mathrm{~d}$ materials $(\mathrm{Fe}, \mathrm{Co}, \mathrm{Ni}$ ) can be produced in various compositions over a wide size regime. However, strongly enhanced total magnetic moments can only be observed in the size regime of very small clusters (less than $2 \mathrm{~nm}$ ), where cooling of the samples to liquid nitrogen or even liquid helium temperatures is required in order to stabilize ferromagnetism. Clusters from magnetic pure rare earth (RE) materials (such as $\mathrm{Gd}, \mathrm{Tb}$, etc.), on the other hand, are extremely reactive and very difficult to handle. Already the production of clean RE clusters under typical pressure conditions in cluster sources will be a major task. Binary alloy clusters, especially combinations of $3 \mathrm{~d}$ metals with $4 \mathrm{~d}$ or $5 \mathrm{~d}$ materials (such as $\mathrm{Pd}, \mathrm{Rh}, \mathrm{Pt}$ ), offer the possibility to stabilize ferromagnetism in clusters at room temperature as has been shown for chemically prepared FePt clusters. Moreover, these clusters can, under certain conditions, be converted into the desired $\mathrm{L} 1_{0}$ structure. This seems to be a starting point for further investigations in this field, that has additional degrees of freedom for building new materials such as the compositions of the cluster and its internal geometric structure. Cluster sources with their chance to form new materials under highly non-equilibrium conditions could be suitable candidates for this kind of research. Recent calculations [196] show that it is even possible to turn typically soft-ferromagnetic materials (such as FeCo alloys) with their high saturation magnetization into hard-magnetic ferromagnets with magnetic anisotropy constants similar to FePt just by introducing a small distortion.

\section{Acknowledgement}

All authors gratefully acknowledge financial support by the European Community in the 5th Framework programme (AMMARE Contract No. G5RD-CT-2001-00478) for this work.

\section{References}

[1] H. Haberland, Clusters of Atoms and Molecules I and II, Springer-Verlag, Berlin, 1995.

[2] U. Kreibig, Optical Properties of Metal Clusters, Springer-Verlag, Berlin, 1995.

[3] K.-H. Meiwes-Broer, Clusters on Surfaces, Springer-Verlag, Berlin, 2000.

[4] H.J. Freund, Surf. Sci. 500 (2002) 271.

[5] H.A. Dürr, S.S. Dhesi, E. Dudzik, D. Knabben, G. van der Laan, J.B. Goedkoop, F.U. Hillebrecht, Phys. Rev. B 59 (1999) R701.

[6] P. Gambardella, S. Rusponi, M. Veronese, S.S. Dhesi, C. Grazioli, A. Dallmeyer, I. Cabria, R. Zeller, P.H. Dederichs, K. Kern, C. Carbone, H. Brune, Science 300 (2003) 1130.

[7] C. Boeglin, S. Stanescu, J.P. Deville, P. Ohresser, N.B. Brookes, Phys. Rev. B 66 (2002) 014439.

[8] M. Bode, O. Pietsch, A. Kubetzka, R. Wiesendanger, Phys. Rev. Lett. 92 (2004) 067201.

[9] R. Röhlsberger, J. Bansmann, V. Senz, K.L. Jonas, A. Bettac, O. Leupold, R. Rüffer, E. Burkel, K.-H. Meiwes-Broer, Phys. Rev. Lett. 86 (2001) 5597.

[10] I.M.L. Billas, A. Châtelain, W.A. de Heer, Science 265 (1994) 1682.

[11] S. Sun, C.B. Murray, D. Weller, L. Folks, A. Moser, Science 287 (2000) 1989.

[12] H.G. Boyen, G. Kästle, K. Zürn, T. Herzog, F. Weigl, P. Ziemann, O. Mayer, C. Jerome, M. Möller, J.P. Spatz, M.G. Garnier, P. Oelhafen, Adv. Funct. Mater. 13 (2003) 359.

[13] G. Kästle, H.G. Boyen, F. Weigl, P. Ziemann, S. Riethmüller, C.H. Hartmann, J.P. Spatz, M. Möller, M.G. Garnier, P. Oelhafen, Phase Transitions 76 (2003) 307. 
[14] H. Brune, Nature 394 (1998) 451.

[15] H. Brune, Surf. Sci. Rep. 31 (1998) 121.

[16] G.M. Francis, L. Kuipers, J.R.A. Cleaver, R.E. Palmer, J. Appl. Phys. 79 (1991) 2942.

[17] K. Lee, J. Callaway, K. Kwong, R. Tang, A. Ziegler, Phys. Rev. B 31 (1985) 1796.

[18] G.M. Pastor, J. Dorantes-Dávila, K. Bennemann, Phys. Rev. B 40 (1989) 7642.

[19] W.A. de Heer, P. Milani, A. Châtelain, Phys. Rev. Lett. 65 (1990) 488.

[20] I.M.L. Billas, J.A. Becker, A. Châtelain, W.A. de Heer, Phys. Rev. Lett. 71 (1993) 4067.

[21] J.P. Bucher, D.C. Douglass, L.A. Bloomfield, Phys. Rev. Lett. 66 (1991) 3052.

[22] D.C. Douglass, J.P. Bucher, L.A. Bloomfield, Phys. Rev. Lett. 68 (1992) 1774.

[23] D.C. Douglass, A.J. Cox, J.P. Bucher, L.A. Bloomfield, Phys. Rev. B 47 (1993) 12874.

[24] A.J. Cox, J.G. Louderback, L.A. Bloomfield, Phys. Rev. Lett. 71 (1993) 1079.

[25] A.J. Cox, J.G. Louderback, L.A. Bloomfield, Phys. Rev. B 49 (1994) 12295.

[26] M.B. Knickelbein, Phys. Rev. Lett. 86 (2001) 5255.

[27] D.P. Pappas, A.P. Popov, A.N. Anisimov, B.V. Reddy, S.N. Khanna, Phys. Rev. Lett. 76 (1996) 4332.

[28] D. Gerion, A. Hirt, I.M.L. Billas, A. Châtelain, W.A. de Heer, Phys. Rev. B 62 (2000) 7491.

[29] D. Gerion, A. Hirt, A. Châtelain, Phys. Rev. Lett. 83 (1999) 532.

[30] M.B. Knickelbein, Chem. Phys. Lett. 353 (2002) 221.

[31] R. Pfandzelter, G. Steierl, C. Rau, Phys. Rev. Lett. 74 (1995) 3467.

[32] K. Wildberger, V.S. Stepanyuk, P. Lang, R. Zeller, P.H. Dederichs, Phys. Rev. Lett. 75 (1995) 509.

[33] C. Binns, Surf. Sci. Rep. 44 (2001) 1.

[34] R.A. Guirado-Lopez, J. Dorantes-Davila, G.M. Pastor, Phys. Rev. Lett. 90 (2003) 226402.

[35] C.P. Bean, J.D. Livingston, J. Appl. Phys. 30 (1959) 120 S.

[36] K. Edmonds, C. Binns, S.H. Baker, M.J. Maher, S.C. Thornton, O. Tjernberg, N.B. Brookes, J. Magn. Magn. Mater. 231 (2001) 113.

[37] J. Bansmann, A. Kleibert, Appl. Phys. A 80 (2005).

[38] H.A. Dür, S.S. Dhesi, E. Dudzik, D. Knabben, G. van der Laan, J.B. Goedkoop, F.U. Hillebrecht, Phys. Rev. B 59 (1999) R701.

[39] Y. Xie, J.A. Blackman, Phys. Rev. B 66 (2002) 085410.

[40] S. Stoyanov, Y. Huang, Y. Zhang, V. Skumryev, G.C. Hadjipanayis, D. Weller, J. Appl. Phys. 93 (2003) 7190.

[41] J. Mühlbach, K. Sattler, E. Recknagel, Surf. Sci. 106 (1981) 18.

[42] H. Pauly, Atom, Molecule and Cluster Beams II, Springer Series on Atomic, Optical, and Plasma Physics, Springer, 2000.

[43] S.H. Baker, S.C. Thornton, K.W. Edmonds, M.J. Maher, C. Norris, C. Binns, Rev. Sci. Instrum. 71 (2000) 3178.

[44] H.V. Tafreshi, P. Piseri, E. Barborini, G. Benedek, P. Milani, J. Nanoparticle Res. 4 (2002) 511.

[45] M.D. Upward, B.N. Cotier, P. Moriarty, P.H. Beton, S.H. Baker, C. Binns, K. Edmonds, J. Vac. Sci. Technol. B 18 (2000) 2646.

[46] C. Binns, M.J. Maher, New J. Phys. 4 (2002) 85.

[47] C. Binns, S.H. Baker, K.W. Edmonds, P. Finetti, M.J. Maher, S.C. Louch, S.S. Dhesi, N.B. Brookes, Physica B (2002) 350.

[48] R.P. Methling, V. Senz, E.D. Klinkenberg, T. Diederich, J. Tiggesbäumker, G. Holzhüter, J. Bansmann, K.H. MeiwesBroer, Eur. Phys. J. D 16 (2001) 173.

[49] R.-P. Methling, Ph.D. thesis, Universität Rostock, Germany, 2004.

[50] V.E. Bondybey, J.H. English, J. Chem. Phys. 74 (1981) 6978.

[51] A.A. Andriotis, M. Menon, Phys. Rev. Lett. 93 (2004) 026402.

[52] G. Ganteför, K.H. Meiwes-Broer, H.O. Lutz, Phys. Rev. A 37 (1988) 2716.

[53] S. Nonose, Y. Sone, K. Onodera, S. Sudo, K. Kaya, J. Phys. Chem. 94 (1990) 2744.

[54] L. Bardotti, B. Prevel, P. Mélinon, A. Perez, Q. Hou, M. Hou, Phys. Rev. B 62 (2000) 2835.

[55] M. Pellarin, B. Baguenard, J.L. Vialle, J. Lermé, M. Broyer, J. Miller, A. Perez, Chem. Phys. Lett. 217 (1994) 349.

[56] J.L. Rousset, A.M. Cadrot, F.S. Aires, P. Mélinon, A. Perez, M. Pellarin, J.L. Vialle, M. Broyer, Surf. Rev. Lett. 1 (1996) 1171.

[57] J.L. Rousset, F.J.C.S. Aires, B.R. Sekhar, P. Mélinon, B. Prevel, M. Pellarin, J. Phys. Chem. B 104 (2000) 5430. 
[58] B. Prevel, J. Lermé, M. Gaudry, E. Cottancin, M. Pellarin, M. Treilleux, P. Mélinon, A. Perez, J.L. Vialle, M. Broyer, Scr. Mater. 44 (2001) 1235.

[59] J. Tuaillon-Combes, M. Négrier, B. Barbara, W. Wernsdorfer, M. Treilleux, P. Mélinon, O. Boisron, A. Perez, Int. J. Nanosci. 2 (2003) 75.

[60] L. Bardotti, B. Prevel, M. Treilleux, P. Mélinon, A. Perez, Appl. Surf. Sci. 164 (2000) 52.

[61] J. Tuaillon, V. Dupuis, P. Mélinon, B. Prevel, M. Treilleux, A. Perez, Philos. Mag. A 76 (1997) 493.

[62] M. Jamet, V. Dupuis, P. Mélinon, G. Guiraud, A. Perez, W. Wernsdorfer, A. Traverse, B. Baguenard, Phys. Rev. B 62 (2000) 493.

[63] P. Jensen, Rev. Mod. Phys. B 71 (1999) 1695.

[64] Q. Hou, M. Hou, L. Bardotti, B. Prevel, P. Mélinon, A. Perez, Phys. Rev. B 62 (2000) 2825.

[65] P. Deltour, P. Jensen, J.L. Barrat, Phys. Rev. Lett. 78 (1997) 4597.

[66] C. Brechignac, P. Cahuzac, F. Carlier, M.D. Fructos, A. Masson, C. Mory, C. Colliex, B. Yoon, Phys. Rev. B 57 (1998) 2084.

[67] H. Haberland, Z. Insepov, M. Moseler, Phys. Rev. B 51 (1995) 11061.

[68] L. Bardotti, B. Prevel, P. Jensen, M. Treilleux, P. Mélinon, A. Perez, J. Gierak, G. Faini, D. Mailly, Appl. Surf. Sci. 191 (2002) 205.

[69] J. Venables, G.D.T. Spiller, M. Hanbücken, Rep. Prog. Phys. 47 (1984) 399.

[70] Y.W. Mo, J. Kleiner, M.B. Webb, M. Lagally, Phys. Rev. Lett. 66 (1999) 1998.

[71] J.P. Bucher, E. Hahn, P. Fernandez, C. Massobrio, K. Kern, Europhys. Lett. 27 (1994) 473.

[72] J.V. Barth, H. Brune, G. Ertl, R.J. Behm, Phys. Rev. B 42 (1990) 9307.

[73] S. Narasimhan, D. Vanderbilt, Phys. Rev. Lett. 69 (1990) 1564.

[74] H. Brune, H. Roeder, C. Boragno, K. Kern, Phys. Rev. B 49 (1994) 2997.

[75] D.D. Chambliss, R.J. Wilson, S. Chiang, J. Vac. Sci. Technol. B 9 (1991) 993.

[76] H. Bulou, C. Goyhenex, Phys. Rev. B 65 (2002) 045407.

[77] I. Meunier, G. Tréglia, J.M. Gay, B. Aufray, B. Legrand, Phys. Rev. B 59 (1999) 10910.

[78] L. Zhang, J. van Elk, U. Diebold, Phys. Rev. B 57 (1998) R4285.

[79] L.P. Nilsen, F. Besenbacher, I. Stensgaard, E. Laegsgaard, C. Engdahl, P. Stoltze, K.W. Jacobsen, J.K. Norskov, Phys. Rev. Lett. 71 (1993) 754.

[80] B. Voigtlander, G. Meyer, N.M. Amer, Phys. Rev. B 44 (1991) 10354.

[81] J.A. Strocio, D.T. Pierce, R.A. Dragosset, P.N. First, J. Vac. Sci. Technol. A 10 (1992) 1981.

[82] I. Chado, F. Scheurer, J.P. Bucher, Phys. Rev. B 64 (2001) 094410.

[83] S. Padovani, I. Chado, F. Scheurer, J.P. Bucher, Appl. Surf. Sci. 42 (2000) 164.

[84] S. Padovani, F. Scheurer, I. Chado, J.P. Bucher, Phys. Rev. B 61 (2000) 72.

[85] G.M. Pastor, Atomic clusters and nanoparticles, in: C. Guet, P. Hobza, F. Spiegelman, F. David (Eds.), Lectures Notes of the Les Houches Summer School of Theoretical Physics, Springer-Verlag, Berlin, 2001.

[86] J.C. Slater, Quantum Theory of Atomic Structure, vol. 1, McGraw-Hill, 1960.

[87] T. Oda, A. Pasquarello, R. Car, Phys. Rev. Lett. 80 (1998) 3622.

[88] M.A. Ojeda, J. Dorantes-Dávila, G.M. Pastor, Phys. Rev. B 60 (1999) 6121.

[89] M.A. Ojeda, J. Dorantes-Dávila, G.M. Pastor, Phys. Rev. B 60 (1999) 9122.

[90] C. Kohl, G.F. Bertsch, Phys. Rev. B 60 (1999) 4205.

[91] G. Rollmann, P. Entel, S. Sahoo, Comput. Mater. Sci., 2004, submitted for publication.

[92] R. Haydock, H. Ehrenreich, F. Seitz, D. Turnbull, Solid State Physics, vol. 35, Academic Press, New York, 1980.

[93] R.H. Victora, L.M. Falicov, S. Ishida, Phys. Rev. B 30 (1984) 3896.

[94] R. Galicia, Rev. Mex. Fis. 32 (1985) 51.

[95] B.V. Reddy, S.N. Khanna, B.I. Dunlap, Phys. Rev. Lett. 70 (1993) 3323.

[96] P. Villasenor-González, J. Dorantes-Dávila, H. Dreyssé, G.M. Pastor, Phys. Rev. B 55 (1997) 15084.

[97] Y. Jinlong, F. Toigo, W. Kelin, Phys. Rev. B 50 (1994) 7915.

[98] Z.-Q. Li, J.-Z. Yu, K. Ohno, Y. Kawazoe, J. Phys.: Condens. Matter 7 (1995) 47.

[99] B. Piveteau, M.-C. Desjonquéres, A.M. Olés, D. Spanjaard, Phys. Rev. B 53 (1996) 9251.

[100] A. Vega, L.C. Balbás, J. Dorantes-Dávila, G.M. Pastor, Phys. Rev. B 50 (1994) 3899.

[101] P. Alvarado, J. Dorantes-Dávila, G.M. Pastor, Phys. Rev. B 58 (1998) 12216. 
[102] P. Strange, Relativistic Quantum Mechanics, Cambridge University Press, Cambridge, 1998.

[103] H.J.F. Jansen, Phys. Rev. B 38 (1988) 8022.

[104] L.D. Landau, E.M. Lifshitz, Quantum Mechanics, vol. 3, Pergamon Press, 1965.

[105] P. Bruno, P.H. Dederichs, P. Grünberg, W. Zinn, Magnetismus von Festkörpern und Grenzflächen of IFF-Ferienkurs, vol. 24, Forschungszentrum Jülich, Jülich, 1993.

[106] G.M. Pastor, J. Dorantes-Dávila, S. Pick, H. Dreyssé, Phys. Rev. Lett. 75 (1995) 326.

[107] F. Bodker, S. Morup, S. Linderoth, Phys. Rev. Lett. 72 (1994) 282.

[108] H. Fritzsche, H.J. Elmers, U. Gradmann, J. Magn. Magn. Mater. 135 (1994) 343.

[109] M. Jamet, W. Wernsdorfer, C. Thirion, D. Mailly, V. Dupuis, P. Mèlinon, A. Pèrez, Phys. Rev. Lett. 86 (2001) 4676.

[110] D. Zitoun, M. Respaud, M.-C. Fromen, M.J. Casanove, P. Lecante, C. Amiens, B. Chaudret, Phys. Rev. Lett. 89 (2002) 037203.

[111] P. Gambardella, A. Dallmeyer, K. Maiti, M.C. Malagoli, W. Eberhardt, K. Kern, C. Carbone, Nature 416 (2002) 301.

[112] K.W. Edmonds, C. Binns, S.H. Baker, S.C. Thornton, C. Norris, J.B. Goedkoop, M. Finazzi, N.B. Brookes, Phys. Rev. B 60 (1999) 472.

[113] T. Koide, H. Miyauchi, J. Okamoto, T. Shidara, A. Fujimori, H. Fukutani, K. Amemiya, H. Takeshita, S. Yuasa, T. Katayama, Y. Suzuki, Phys. Rev. Lett. 87 (2001) 257201.

[114] J.T. Lau, A. Frölisch, R. Nietubyc, M. Reif, W. Wurth, Phys. Rev. Lett. 89 (2002) 057201.

[115] B. Nonas, I. Cabria, R. Zeller, P.H. Dederichs, T. Huhne, H. Ebert, Phys. Rev. Lett. 86 (2001) 2146.

[116] I. Cabria, B. Nonas, R. Zeller, P.H. Dederichs, Phys. Rev. B 65 (2002) 054414.

[117] S.E. Apsel, J.W. Emmert, J. Deng, L.A. Bloomfield, Phys. Rev. Lett. 76 (1996) 1441.

[118] M. Castro, C. Jamorski, D. Salahub, Chem. Phys. Lett. 271 (1997) 133.

[119] B.V. Reddy, S.K. Nayak, S.N. Khanna, B.K. Rao, P. Jena, J. Phys. Chem. A 102 (1998) 1748.

[120] F.A. Reuse, S. Khanna, Eur. Phys. J. D 6 (1999) 77.

[121] S. Foner, Rev. Sci. Instrum. 30 (1959) 548.

[122] C. Binns, M.J. Maher, Q.A. Pankhurst, D. Kechrakos, K.N. Trohidou, Phys. Rev. B 66 (2002) 184413.

[123] J. Kerr, Philos. Mag. 3 (1877) 321.

[124] E.R. Moog, S.D. Bader, Superlatt. Microstruct. 1 (1985) 543.

[125] Z.J. Yang, M.R. Scheinfein, J. Appl. Phys. 74 (1993) 6810.

[126] Z.Q. Qiu, S.D. Bader, J. Magn. Magn. Mater. 200 (1999) 664.

[127] J. Bansmann, V. Senz, R.P. Methling, R. Röhlsberger, K.-H. Meiwes-Broer, Mater. Res. Eng. C 19 (2002) 305.

[128] G. Panaccione, F. Sirotti, G. Rossi, Solid State Commun. 113 (2000) 373.

[129] J. Bansmann, L. Lu, K.-H. Meiwes-Broer, T. Schlathölter, J. Braun, Phys. Rev. B 60 (1999) 13860.

[130] C. Roth, F.U. Hillebrecht, H.B. Rose, E. Kisker, Phys. Rev. Lett. 70 (1994) 3479.

[131] F. Sirotti, S. Girlando, P. Prieto, L. Floreano, G. Panaccione, G. Rossi, Phys. Rev. B 61 (2000) R9221.

[132] P. Elleaume, J. Synchrotron Radiat. 19 (1994) 1.

[133] G. Schütz, W. Wagner, W. Wilhelm, P. Kienle, Phys. Rev. Lett. 58 (1987) 737.

[134] B.T. Thole, P. Carra, F. Sette, G. van der Laan, Phys. Rev. Lett. 68 (1992) 1943.

[135] P. Carra, B.T. Thole, M. Altarelli, X. Wang, Phys. Rev. Lett. 70 (1993) 694.

[136] J. Stöhr, H. König, Phys. Rev. Lett. 75 (1995) 3748.

[137] G. van der Laan, Phys. Rev. B 57 (1998) 5250.

[138] C.T. Chen, Y.U. Idzerda, H.J. Lin, N.V. Smith, G. Meigs, E. Chaban, G.H. Ho, E. Pellegrin, F. Sette, Phys. Rev. Lett. 75 (1995) 152.

[139] R. Nakajima, J. Stöhr, Y.U. Idzerda, Phys. Rev. B 59 (1999) 6421.

[140] J. Stöhr, NEXAFS Spectroscopy, Springer Series in Surface Sciences, vol. 25, Springer, Heidelberg, 1992.

[141] K. Fauth, Appl. Phys. Lett. 85 (2004) 3271.

[142] T.J. Regan, H. Ohldag, C. Stamm, F. Nolting, J. Lüning, J. Stöhr, R.L. White, Phys. Rev. B 64 (2001) 214422.

[143] S. Gota, M. Gautier-Soyer, M. Sacchi, Phys. Rev. B 62 (2000) 4187.

[144] K. Fauth, private communication.

[145] J.B. Kortright, S.K. Kim, Phys. Rev. B 62 (2000) 12216.

[146] H.C. Mertins, D. Abramsohn, A. Gaupp, F. Schäfers, W. Gudat, O. Zaharko, H. Grimmer, P.M. Oppeneer, Phys. Rev. B 66 (2002) 184404. 
[147] C.C. Kao, J.B. Hastings, E.D. Johnson, D.P. Siddons, G.C. Smith, G.A. Prince, Phys. Rev. Lett. 65 (1990) 373.

[148] D. Attwood, Soft X-Rays and Extreme Ultraviolet Radiation, Cambridge University Press, Cambridge, 1999.

[149] C.C. Kao, C.T. Chen, E.D. Johnson, J.B. Hastings, H.J. Lin, G.H. Ho, G. Meigs, J.M. Brot, S.L. Hulbert, Y.U. Idzerda, C. Vettier, Phys. Rev. B 50 (1994) 9599.

[150] S.W. Lovesey, S.P. Collins, X-Ray Scattering and Absorption by Magnetic Materials, Clarendon Press, Oxford, 1996.

[151] R. Felix-Medina, J. Dorantes-Davila, G.M. Pastor, Phys. Rev. B 67 (2003) 094430.

[152] S.H. Baker, C. Binns, K.W. Edmonds, M.J. Maher, S.C. Thornton, S. Louch, S.S. Dhesi, J. Magn. Magn. Mater. 247 (2002) 19.

[153] B.G. Johnson, P.J. Berlowitz, D.W. Goodman, Surf. Sci. 217 (1989) 13.

[154] H. Fritzsche, J. Kohlhepp, U. Gradmann, Phys. Rev. B 51 (1995) 15933.

[155] H. Pinkvos, H. Poppa, E. Bauer, J. Hurst, Ultramicroscopy 47 (1992) 339.

[156] M. Getzlaff, J. Bansmann, J. Braun, G. Schönhense, J. Magn. Magn. Mater. 161 (1996) 70.

[157] U. Gradmann, G. Waller, Surf. Sci. 116 (1982) 539.

[158] H.J. Elmers, U. Gradmann, Appl. Phys. A 51 (1990) 255.

[159] J. Bansmann, et al., to be published.

[160] Y. Xie, J.A. Blackman, Phys. Rev. B 63 (2001) 125105.

[161] Y. Xie, J.A. Blackman, Phys. Rev. B 64 (2001) 195115.

[162] Y. Xie, J.A. Blackman, Phys. Rev. B 66 (2002) 155417.

[163] J. Guevara, F. Parisi, A.M. Llois, M. Weissmann, Phys. Rev. B 55 (1997) 13283.

[164] J.A. Franco, A. Vega, F. Aguilera-Granja, Phys. Rev. B 60 (1999) 434.

[165] C. Paduani, J.C. Krause, J. Appl. Phys. 86 (1999) 199, and references therein.

[166] R.H. Victora, L.M. Falicov, Phys. Rev. B 30 (1984) 259.

[167] J. Izquierdo, D. Bazhanov, A. Vega, V.S. Stepanyuk, W. Hergert, Phys. Rev. B 63 (2001) 140413.

[168] B. Lazarovits, L. Szunyogh, P. Weinberger, Phys. Rev. Lett. 65 (2002) 104441.

[169] J. Kohlhepp, U. Gradmann, J. Magn. Magn. Mater. 139 (1995) 347.

[170] J. Dorantes-Dávila, H. Dreyssé, G.M. Pastor, Phys. Rev. Lett. 91 (2003) 197206.

[171] J.A.C. Bland, B. Heinrich (Eds.), Ultrathin Magnetic Structures I and II, Springer-Verlag, Berlin, 1994.

[172] J. Dorantes-Dávila, G.M. Pastor, Phys. Rev. Lett. 81 (1998) 208.

[173] R. Felix-Medina, J. Dorantes-Davila, G.M. Pastor, New J. Phys. 4 (2002) 100.

[174] V. Heine, in: H. Ehrenreich, F. Seitz, D. Turnbull (Eds.), Solid State Physics, vol. 35, Academic Press, New York, 1980

[175] C.G. Zimmermann, M. Yeadon, K. Nordlund, J.M. Gibson, R.S. Averback, U. Herr, K. Samwer, Phys. Rev. Lett. 83 (1999) 1163.

[176] S. Padovani, F. Scheurer, J.P. Bucher, Europhys. Lett. 45 (1999) 327.

[177] O.V. Lysenko, V.S. Stepanyuk, W. Hergert, J. Kirschner, Phys. Rev. Lett. 89 (2002) 126102.

[178] S. Padovani, I. Chado, F. Scheurer, J.P. Bucher, Phys. Rev. B 59 (1999) 11887.

[179] V. Russier, C. Petit, J. Legrand, M.P. Pileni, Phys. Rev. B 62 (2000) 3910.

[180] N. Pinna, M. Maillard, A. Courty, V. Russier, M.P. Pileni, Phys. Rev. B 66 (2002) 045415.

[181] D.M. Eigler, E.K. Schweizer, Nature 344 (1990) 524.

[182] H. Roeder, E. Hahn, H. Brune, J.P. Bucher, K. Kern, Nature 366 (1993) 141.

[183] P. Ohresser, N.B. Brookes, S. Padovani, F. Scheurer, H. Bulou, Phys. Rev. B 64 (2001) 104429.

[184] J. Shen, R. Skomski, M. Klaua, H. Jenniches, S.S. Manoharan, J. Kirschner, Phys. Rev. B 56 (1997) 2340.

[185] J.W. Lee, J.R. Jeong, S.C. Shin, J. Kim, S.K. Kim, Phys. Rev. B 66 (2002) 172409.

[186] T.J. Jackson, C. Binns, E.M. Forgan, E. Morenzoni, C. Niedermayer, H. Glückler, A. Hofer, H. Luetkens, T. Prokscha, T.M. Riseman, A. Schatz, M. Birke, J. Litterst, G. Schatz, H.P. Weber, J. Phys.: Condens. Matter 12 (2000) 1399.

[187] D. Kechrakos, K.N. Trohidou, J. Magn. Magn. Mater. 262 (2003) 107.

[188] P. Allia, M. Coisson, P. Tiberto, F. Vinai, M. Knobel, M.A. Novak, W.C. Nunes, Phys. Rev. B 64 (2001) 144420.

[189] E.M. Chudnovsky, J. Magn. Magn. Mater. 40 (1983) 21.

[190] E.M. Chudnovsky, W.M. Saslow, R.A. Serota, Phys. Rev. B 33 (1986) 251.

[191] W.M. Saslow, Phys. Rev. B 35 (1987) 3454.

[192] E.M. Chudnovsky, J. Appl. Phys. 64 (1988) 5770. 
[193] E.M. Chudnovsky, in: J.A. Fernandez-Baca, W.-Y. Ching (Eds.), The Magnetism of Amorphous Metals and Alloys, Springer Series Solid-State Sciences, vol. 80, World Scientific, Singapore, 1995.

[194] J.F. Löffler, H.B. Braun, W. Wagner, Phys. Rev. Lett. 85 (2000) 1990.

[195] D.J. Sellmyer, M. Yu, R.D. Kirby, Nanostruct. Mater. 12 (1999) 1021.

[196] T. Burkert, L. Nordström, O. Eriksson, O. Heinonen, Phys. Rev. Lett. 93 (2004) 027203.

[197] VACOFLUX 50, Product of Vacuumschmelze GmbH, Hanau, Germany.

[198] M. Getzlaff, A. Kleibert, R.P. Methling, J. Bansmann, K.-H. Meiwes-Broer, Surf. Sci. 566-568 (2004) 332.

[199] M. Farle, A. Berghaus, Y. Li, K. Baberschke, Phys. Rev. B 42 (1990) 4873.

[200] D. Sander, C. Schmidthals, A. Enders, J. Kirschner, Phys. Rev. B 57 (1998) 1406.

[201] C. Schmidthals, D. Sander, A. Enders, J. Kirschner, Surf. Sci. 417 (1998) 361.

[202] H. Ebert, M. Battocletti, Solid State Commun. 98 (1996) 785.

[203] V. Dupuis, L. Favre, S. Stanescu, J. Tuaillon-Combes, E. Bernstein, A. Perez, J. Phys.: Condens. Matter 16 (2004) S2231.

[204] M. Jamet, W. Wernsdorfer, C. Thirion, V. Dupuis, P. Mélinon, A. Perez, D. Mailly, Phys. Rev. B 69 (2004) 024401.

[205] F.J.A. den Broeder, W. Hoving, P.J.H. Bloemen, J. Magn. Magn. Mater. 93 (2002) 562.

[206] M. Jamet, M. Negrier, V. Dupuis, J. Tuaillon-Combes, P. Mélinon, A. Perez, W. Wernsdorfer, B. Barbara, B. Baguenard, J. Magn. Magn. Mater. 237 (2002) 293.

[207] V. Dupuis, M. Jamet, J. Tuaillon-Combes, L. Favre, S. Stanescu, M. Treilleux, E. Bernstein, P. Mélinon, A. Perez, Recent research developments in magnetism and magnetic materials, Transworld Res. Netw. 1 (2003) 101.

[208] S. Dennler, J. Morillo, G.M. Pastor, Surf. Sci. 532-535 (2003) 334.

[209] S. Dennler, J. Morillo, G.M. Pastor, J. Phys.: Condens. Matter 16 (2004) S2263.

[210] G. Kresse, J. Hafner, Phys. Rev. B 47 (1993) 558.

[211] G. Kresse, J. Furthmüller, Phys. Rev. B 54 (1996) 11169.

[212] M. Munoz-Navia, J. Dorantes-Dávila, G.M. Pastor, J. Phys.: Condens. Matter 16 (2004) S2251.

[213] M.-C. Fromen, P. Lecante, M.J. Casanove, P.B. Guillemaud, D. Zitoun, C. Amiens, B. Chaudret, M. Respaud, R.E. Benfield, Phys. Rev. B 69 (2004) 235416.

[214] M. Respaud, private communication.

[215] H. Takeshita, Y. Suzuki, H. Akinaga, W. Mitzutani, K. Ando, T. Katayama, A. Itoh, K. Tanaka, J. Magn. Magn. Mater. 165 (1997) 38.

[216] J. Xu, M.A. Howson, B.J. Hickey, D. Greig, E. Kolb, P. Veillet, N. Wiser, Phys. Rev. B 55 (1997) 416.

[217] O. Toulemonde, J.P. Bucher, Surf. Sci., to be published.

[218] H.J. Elmers, J. Hauschild, H. Höche, U. Gradmann, H. Bethge, D. Heuer, U. Köhler, Phys. Rev. Lett. 73 (1994) 898.

[219] J.P. Bucher, I. Chado, H. Bulou, P. Ohresser, Phys. Rev. Lett. 90 (2003) 149703.

[220] R. Allenspach, M. Stampanoni, A. Bischof, Phys. Rev. Lett. 65 (1990) 3344.

[221] I. Chado, C. Goyhenex, H. Bulou, J.P. Bucher, Phys. Rev. B 69 (2004) 085413.

[222] C.H. Hee, Y.Y. Zou, J.P. Wang, J. Appl. Phys. 91 (2002) 8004.

[223] B. Ujfalussy, L. Szunyogh, P. Bruno, P. Weinberger, Phys. Rev. Lett. 77 (1996) 1805.

[224] I. Chado, J.P. Bucher, D. Kechrakos, K. Trohidou, J. Phys.: Condens. Matter 16 (2004) S2287.

[225] K. Binder, D.W. Heermann, Monte Carlo Simulation in Statistical Physics, Springer Series in Solid-State Sciences, vol. 80, Springer-Verlag, Berlin, 1998.

[226] K. Yaldram, K. Binder, J. Stat. Phys. 62 (1991) 161.

[227] G. Tréglia, B. Legrand, F. Ducastelle, Europhys. Lett. 7 (1988) 575.

\section{Glossary}

ACIS: arc cluster ion source

$A F M$ : atomic force microscopy

BESSY: Berliner Elektronen Speicherring Gesellschaft für Synchrotronstrahlung

$C S S G$ : correlated super-spin glass

$E D X$ : energy dispersive $\mathrm{X}$-ray spectroscopy

$E_{F}$ : Fermi energy 
EXAFS: extended X-ray absorption fine structure

$F C / Z F C$ : field cooled/zero field cooled

$F I B$ : focused ion beam

$H_{c}$ : coercive field

$H O P G$ : highly oriented pyrolitic graphite

HRTEM: high-resolution transmission electron microscopy

ISS: ion scattering spectroscopy

LDOS: local density of states

$\angle E C B D$ : low-energy cluster beam deposition

$\angle E E D$ : low-energy electron diffraction

$M A E$ : magnetic anisotropy energy

$M C$ : Monte Carlo

MLDAD: magnetic linear dichroism in the angular distribution of photoelectrons

MOKE: magneto-optical Kerr effect

$M_{r}:$ remanent magnetization

$M_{s}:$ saturation magnetization

$N N$ : nearest-neighbour

$R A$ : random anisotropy

$R E$ : rare earth

RHEED: reflection high-energy electron diffraction

RBS: Rutherford backscattering

$S O$ : spin-orbit (interaction)

SQUID: superconducting quantum interference device

STM: scanning tunneling microscopy

$T B$ : tight binding

TEM: transmission electron microscopy

$T E Y$ : total electron yield

$T M$ : transition metals

TOF: time-of-flight spectroscopy

$U H V$ : ultrahigh vacuum

VSM: vibrating sample magnetometry

$X A S$ : X-ray absorption spectroscopy

$X M C D$ : X-ray magnetic circular dichroism in photoabsorption

$X P S: \mathrm{X}$-ray photoelectron spectroscopy

$X U V$ : extreme ultraviolet 